33,24

\title{
A Proposal for a
}

Positron-Electron Colliding-Beam Storage Ring Project (PEP)

LBL Report No. 2688

SLAC Report No. 171

April 1974

Under Contract with the U. S. Atomic Energy Commission

Contract W-7405-ENG-48

Contract AT(04-3)-515 
SIAC $\cdots 171$

(T/E/A)

$\mathrm{UC}-28$

A PROPOSAL FOR A

POSITRON-ELECTRON COLLIDING-BEAM STORAGE RING PROJECT

(PEP)

STANFORD LINEAR ACCELERATOR CENTER

STANFORD UNIVERSTTY

Stanford, California 94305

and

NOTICE

This repont was prepared as an eccount of work uponsored by the United Staten Govern, sent. Netthar the United States nor the United Staten Atomic Enargy Commistion, nor any of thetr employes, nor any of

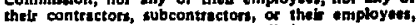
mekes any warranty, expreas or implisd, or anumes any makl lebility or sespondblity fot the eccuracy, com. pletenes of usefunew of any iaformation. apparatus. product on procese dicloued, or teptemats thit its use mould not infringe privatoly owned tights.

LAWRENCE BERKELEY LABORATORY

UNIVERSTTY OF CALIFORNIA

Berkeley, California 94720

PREPARED FOR THE U.S. ATOMIC ENERGY

COMMISSION UNDER CONTRACTS NO. AT (04-3) -515

AND NO. W-7405-ENG-48

April 1974

Printed in the United States of America. Avallable from National Technical Information Service, U.S. Department of Commerce, 5285 Port Royal Road, Springfield, Virginia 22151. Price: Printed copy \$5.45. 


\section{PROPOSAL}

It is proposed that the United States Atomic Energy Conmission support the constructica jointly by the Lawrence Berkeley Laboratory and the Stanford Linear Accelerator Center of a 15-GoV electron-positron colliding-beam facility at the Stanford Linear Accelerator Center.

This proposal was prepared by the stafis of the Lawrence Berkeley Laboratory and the Stanford Linear Accelerator Center. 


\section{PREFACE}

In presenting this joint propogal for a large colliding-beam facility, the Lawrence Berkeley Laboratory (LBL) and the Stanford Linear Accelerator Center (SLAC) have responded to the great importance of the colliding-beam technique for reaching otherwise unattainable reaction energfes. The physles possibilities offered by colliding beams are attracting growing enthusiasm from both experimental and theoretical physicists. The successes already posted by colliding-beam storage rings in the U.S., in Europe and in the U.S.S.R., and especially by the SPEAR electron-positron project at SLAC, have established at once the feasibllity of designing and bullding such machines on a large scale and the practicality of doing physics experiments with them. The proposed storage ring (PEP), operating at beam energles of $15 \mathrm{GeV}$, will make collisions ytelding an interaction energy of $30 \mathrm{GeV}$ in the center-of-mass which is greater than the interaction energy from $400-\mathrm{GeV}$ protons from either the CERN II or NAL. acselerators, the two largest scientific instruments ever constructed. By comparison, the cost effectiveness of colliding-beam facilities is most impressive.

This would be the third storage ring project to be constructed at Stanford. The first, the 500-MeV Princeton-Stanford electron-electron storage rings, performed the first high-energy experiment with colliding beams. The second, the SPEAR facility now being upgraded to a beam energy of $4.2 \mathrm{GeV}$, is providing new regults with profound implications for elementary particle theory. Many of the design details of the facllity proposed here are based on SPEAR designs and experience. The success of SPEAR establishes the technical feasiblitity of the proposed facility and also provides a strong basis for confidence in the cost estimates. 
The SLAC and LBL accelerator groups together comprise a highly complementary group with technical expertise and capability that is well matched to the requirements of the PEP project. While a successful joint study has been under way for the past two years, more recently a formal agreement for the continued collaborative effort in the construction and operational stages of PEP have been negoitiated. The Regents of the University of Calffornia and the Trustees of Stanîferd Univergity have given approval for this cooperative venture.

The facility described in this proposal is designed to be compatible with the later addition of a superconducting proton storage ring capable of storing 200$\mathrm{GeV}$ protons for collision with the $15-\mathrm{GeV}$ electrons or positrons. The design also permits the installation of a second electron-positron storage ring to furnish the capability of electron-electron collisions and positron-positron collisions If that should prove desirable. Neither of these additional rings precludes the other; i.e., both could occupy the tunnel at once. The physics potential of these improvement programs fully warrants providing compatibility.

In proposing to construct this storage ring at SLAC, advantage is being taken of the availability there of the very-high-energy, high-power electron accelerator with its attendant high-current positron beam. The two-mile accelerator is a uniquely suitable facility for meeting the filling requirements of a high-energy electron-positron storage ring like PEP. 


\section{TABLE OF CONTENTS}

I. INTRODUCTION

A. Hitory of the Project 1

B. High `nergy Physics with Electron-Positron Colliding 2 Beaw 18

2

C. Description of the PEP Facillty 6

D. Other Electron-Positron Colliding-Beam Facllities 9

E. Cost -3timate and Time Schedule 11

II. PHYSICS WTH ELECTRON-POSITRON COLLIDING BEAMS 12

A. Introduction $\quad 12$

B. Aunlhilation into Hadrone 13

C. Weak Interastions and New Particle Production 21

D. Tests of Quantum Electrodynamics (QED) 25

E. Two-Photon Procesees 26

F. Unique Features of $\mathrm{e}^{+} \mathrm{e}^{-}$Colliding Beams 30

III. DESCRIPTION OF THE ELECTRON-POSITRON STORAGE RING $\quad 32$

A. Magnetic Focusing Syatem for the Storage Ring 32

$\begin{array}{ll}\text { B. Rf System } & \mathbf{4 7}\end{array}$

C. Vacuum $\quad 59$

D. PEP Injection $\quad 68$

E. Instrumentation Monitoring and Control 77

$\begin{array}{ll}\text { IV. PHYSICAL PIANT } & 81\end{array}$

A. Beam Housing and Site Improvement 81

$\begin{array}{ll}\text { B. Shlelding Requirements } & 87\end{array}$ 
C. Experimental Areas $\quad 90$

D. Utilities 99

V. COST ESTIMATES AND SCHEDULE 102

APPENDIX A : The Proton-Electron System 111

APPENDIX B : Summary of Soll Studies to Date 128

APPENDIX C : Copperative Basic Agreement between the Board 130 of Trustees of the Leland Stanford Junior University and the Regents of the University of California 


\section{LIST OF FIGURES}

Page

1. Layout of the PEP ring superimposed on an aerial view of the SLAC site.

2. Electron-positron colliding-beam facilities of the world.

3. Electron and positron annihilating via the one-photon ( $\left.\gamma^{*}\right)$ intermediate state into a final state of many hadrons.

4. Proton-proton collision with constituent particles a and b interacting to produce the observed hadron at large $p_{\perp}$.

5. Electron-positron annihilation into parton and anti-parton with the parton and anti-parton decaying into the final hadron jets.

6. Quantum electrodynamical processes.

7. Electron-positron scattering and producing hadrons via the two-photon intermediate state.

8. Design luminosity as a function of veam energy showing the nominal operating range and the upper limit as limited by $r f$ power.

9. Component layout of a standard cell. Dimenrions are in meters.

10. Ths straight insertion, which is symmetric about the interaction point, is shown from the centerline of the cell quaduupole to the interaction point. Dimensions are in meters.

11. Bending magnet for the electron-positron storage ring.

12. Standard 127-mm-bore quadrupole used in the bending cells.

13. Large-bore quadrupole used near the interaction regions of the insertions. 
14. Five-cavity $\pi$-mode accelerating structure used for the SPEAR II rf system and proposed for PEP.

15. Power supply arrangement for a typical klystron group showing the voltage control system.

16. Block diagram for the drive, phasing and cavity tuning systems.

17. Cross section of the vacuum chamber for the bending magnets. The distributed ion pump which operates in the fringing field region of the magnet is shown with one of the high-voltage terminals.

18. Power flux due to synchrotron radiation plotted for one-half oi a 63 standard cell. The discontinuities are caused by discontinuities in the bending field and by changes in the curvature of the vacuum chamber.

19. The photon energy spectrum $d^{2} N /(d \epsilon d t)$ per milliampere of 64 stored current is plotted as a function of photon energy. The spectral power $\mathrm{dP} / \mathrm{d} \epsilon$ per milliampere is also shown. Both curves are for $15 \mathrm{GeV}$.

20. Pressure distribut, on in a standard cell with two $100-\mathrm{mA}$ stored beams. Pumping is provided by $100 \mathrm{l} / \mathrm{sec}$ holding pumps and $1200 \mathrm{\ell} / \mathrm{sec}$ distributed ion pumps located as shown.

21. Filling ti.ne vs injection energy. Filling time is the time required to fill both beams to a current of $100 \times[\mathrm{E}(\mathrm{GeV}) / 15] \mathrm{mA}$ from a beam of $20 \mathrm{~mA} \mathrm{e}^{+}$and $40 \mathrm{~mA} \mathrm{e}^{-}$in a $1.4 \mathrm{nsec}$ pulse with $50 \%$ capture efficiency.

22. Injection system beam transport lines. 
23. : rizontal betatron phase plot showing septum lucation.

24. Schematic of combined kicker-septum magnet. The injected beam is kicked in the opposite direction from the stored beam. All conductors are assumed to be the same thickness and operate with the same current density. They are about $2 \mathrm{~m}$ long and use a pulse of approximately 5000 amperes for $15-\mathrm{GeV}$ injection.

25. PEP region numbering scheme. The accelerator beam is defined to run from west to east with Region 12 on the north side.

26. Cross section of the PEP housing.

27. "Standard" interaction area with alcoves.

23. "Standard Plus" interaction area with a 30-m pit.

29. "Big I" interaction area for a large magnetic detector.

30. View of an experimental hs": showing shielding coufiguration proposed for $e^{+} \mathrm{e}^{-}$rings.

A1. Schematic view of the PEP electron-proton ring configuration.

A2. Schematic side view of insertion and cell structure for the proton-electron system.

A3. Cross sections of the normal-cell superconducting dipole and quadrupole magnets.

A4. Tunnel cross sections with electron and proton magnets in alternate sextants of the ring. 


\section{LIST OF TABLES}

Page

1. Available reaction energy from various high-energy machines.

2. General parameters and lattice parameters of the PEP electronpositron colliding-beam storage ring.

3. Typical beam sarameters of PEP.

4. Rf system parameters. 


\section{INTRODUCTION}

\section{A. History of the Project}

In the summer of 1971, a group of physicists working at LBL and SLAC, including visitors from CERN and Frascati, Italy, began an inquiry into the feasibility of a new colliding-beam complex cupable of producing collisions at higher energies than hitherto envisaged between electrons and positrons and also between electrons and protons and positrons and protons. They concluded that such a facility, which would consist of an electron storage ring and a proton storage ring, was quite feasible and that no known physical limitation of the behavior of stored beams would prevent the achievement of luminosities sufficient to yield useful reaction rates for many important high-energy-physics processes. To illustrate these conclusions, a conceptual design example was described and analyzed. 1

Subsequently, the collaborative study between LBL and SLAC grew, involving more people as more refined concepts and designs were devised, and a combination of a 15-GeV electron-positron ring and a superconducting proton ring emerged as the preferred design. It was dubbed PEP. ${ }^{2,3}$ In the meantime, other laboratories began studying similar or related ideas in both the U.S. and Europe, and their representatives came together at SLAC and LBL in the summer of 1973 to exchange and compare ideas.

By this time, there was a consensus that the electron-positron storage ring component of the system, operated at beam energies up to 15 to $20 \mathrm{GeV}$ and capable of yielding high lurninosity in electron-positron collisions, was a straightforward extension of techniques already successfully used in several laboratories and that such a ring could be designed and built immediately with confidence. For the proton ring, supercondicting-magnet technology offered 
the promise of achieving high beam energy with economical aize and with low power consumption; however there were some technical uncertainties yet to be resolved.

In the meantime, electron-pontron rings operating in Europe and the U.S. had rovealed that a wealth of new and previously unexpected high-energy physics information concerning the atructure of elementary particles, both leptons and hadrons, was forthcoming from electron-positron collisions. These experiments have shown that it is urgent to move to higher energles than those avallable from existing machines.

With these facts in mind, LBL and SLAC jointly decided to propose the immodiate design and construction of the 15-GeV electron-positron storage ring and to defer the proposal of the proton storage ring until further development of aperconducting technology had taken place. The two laboratories agreed to locate PEP at SiAC and to design the electron-positron ring and its housing to be compatible with the future addition of a $200-\mathrm{GeV}$ proton ring such as that described in Appendix $A$ of this report. The two universittes aigned an agreement in February, 1974, outlining joint financial and management arrangements for the project. The text of that agreement appears in Appendix $\mathrm{C}$ of this proposal.

\section{B. High Enerrsy Physics with Eleatron-Posttron Colliding Beams}

The current generation of htgh-energy electron-positron colliding-beam storage rings opened up a new phyotcal reglon for the study of elementary particles and their interactions, the region in which a state of pure energy is produced by the annibilation of the colliding electron and positron. Thts state coines mto being only when a particle strikes its anti-particle and therefore does not occur when primary beams from conventional accelerators strike material 
targets or when protons collide with protons in a proten-proton storage ring gystem. The energy can rematerialize into combinations of all of the presently known elementary particles. Thus data can be obtained about the structure and interactions of these particles in a now experimental regime.

The results from entering this new region have been surpriging and profound. As the energies of the colliding beams have been increased, the results of experiments done with them have become more and more difficult to understand in terms of present models of elementary-particle structure and interactions. Most recently, new experiments from the SPEAR facllity at SLAC and the CEA factlity in Cambridge have given results which do not confirm the predictione of the theoretical ideas involving substructure within the nucleon which had been so successful in explaining a host of experiments done with conventional accelerators. The resolution of thie contradiction seems certain to lead to a far deeper understanding of elementary-particle physics.

With the PEP storage ring we are proposing to extend the energy parameter in electron-positron collisions to $30 \mathrm{GeV}$ of avallable reaction energy, thus greatly expanding our reach into the annihilation region, by bullding a storage ring which will contain counter-circulating beams of energies up to $15 \mathrm{GeV}$ each. The available reaction energy will be comparable to that obtainable at the world' $s$ highest-energy accelerators, even though the energy of the circulating beams is very much lower. This is true because, when two oppositely directed antiparticle; of the same mcmentum collide, the total energy, i.e., the sum of their energies, is avaflable to the reaction. In contrast, when a beam particle from a conventional accelerator strikes a stationary target particle, most of its energy is necessarily tied up in the continuing forward motion of the reaction products, as required by the law of conservation of momentum, and only a small fraction 
Is avallable to the reaction. This fact is illuatrated in Table 1 below, which show the avallable reaction energy for the PEP electron-posttron ring and for the three highest-energy machines now operating or under construction, including the world's only proton-proton colliding-beain device, the CERN Intersecting Storage Ringe (ISR). The most Important conclusion to be drawn from the table is that the PEP facility will provide avallable reaction energies coverIng almost the entire range covered by other machines, and covering it through the annihilation process in which the intermediate state is one of pure energy and is inaccessible through the other machines.

\section{Table 1}

Available reaction energy from various high-energy machines

\begin{tabular}{cc}
\hline Machine & $\begin{array}{c}\text { Avallable } \\
\text { Reactlon Energy }\end{array}$ \\
\hline PEP (15-GeV e $\mathrm{e}^{-}$storage ring) & $30 \mathrm{GeV}$ \\
NAL (400-GeV proton accelerator) & $27.4 \mathrm{GeV}$ \\
CERN II (400-GeV proton accelerator) & $27.4 \mathrm{GeV}$ \\
ISR (28-GeV p-p storage ring) & $56 \mathrm{GeV}$ \\
\hline
\end{tabular}

It is interesting to wote that an electron accelerator capable of proctucing the same available reaction energy as PEP by striking stationary target electrons with high-energy positrons would have to accelerate the positrons to almost a million GeV, about forty thousand times the energy of the SLAC twomile accelerator.

The range of experimental studies opened up by PEP is extremely rich and varied, spanning the entire field of elementary-particle physics from the strong 
interactions through the electromagnetic interactions to the weak interactions. In the fteld of strong interactions, reactions leading to mesons and nucleons in the final state will reveal new and vital information about the structure and substructure of the elementary particles. For example a conceptually simple experiment, the measurement of the total reaction probablity to produce strongly interacting particles by electron-posttron collisions, tests some very basic hypotheses about the structure of the particles produced. These hypotheses have falled the tests of experiments with the present generation of electronpositron rings, and they may or may not become applicable at higher energies. On the other hand, experiments at higher energies may demand entirely new theoretical constructs.

In the field of pure electromagnetism we can study processes with only electrons, mu-mesons and gamma rays as reaction products. The theory of the electromagnetic interaction, quantum electrodynamics, is the only successful field theory in particle physics in the sense that all experimental tests to date agree with the predictions of this theory. PEP can greatly increase the energy limits to which this theory can be tested. Partioularly exciting ts the fact that, if present trends in the strong interactions obgerved in $\mathrm{e}^{+} \mathrm{e}^{-}$colliding beams continue to the maximum PEP energy, and if our present understanding of the way these reactions take place has any relation to physical reality, then cuantum electrodynamics must break down in the PEP energy region.

PEP will open wide new vistas for the study of weak interactions via electron-positron collisions. For example, the calliding electron-positron patr can transform itself into a mu-meson pair by both weak and electromagnetic interactions, and the energy-dependences of these two processes are such that the weak interaction becomes more and more competitive with the electromagnetic 
at higher energles, and, at PEP energies, the interference between the two should become observable. Particle physicists are now seeking a unified picture of the weak and electromagnetic interactions and PEP offers the possibility of testing these concepts from a new experimental vantage point.

These experimental opportunities and others are discussed in more detail in Section II.

In summary, the proposed high-energy electron-positron colliding-beam facility PEP offers the possiblitity of the study of a very broad range of fundamental questions in particls physics in a new and presently inaccessible energy region. The mysteries unvelled in the present generation of electron-positron colliding-beam factlities lead us to expect new phenomena to be uncovered with this device. These experiments, together with the complementary experiments with protons, neutrinos and mesons at the highest-energy proton accelerators, offer great promise of leading to a now depth of understanding of elementary particles and the fundamental laws of physics.

\section{Description of the PEP Facility}

The main component of the proposed facility is a storage ring in which beams of positrons and electrons circulate in opposite directions in a vacuum chamber located in a magnetic guide field having six bending arcs and six long straight sections. The major diameter of the ring is about $700 \mathrm{~m}$ and the radius of the $\operatorname{arcs}$ is about $220 \mathrm{~m}$. The facility is shown in Fig. 1. The electrons and positrons are produced in the SLAC linac and are introduced into the storage ring via two beam transport paths emanating from the end of the two-mile accelerator and joining the storage ring in the northwest and southwest straight sections. Beams of energies up io $15 \mathrm{GeV}$ can be stored, and, at a future date, components could be added to permit energies as high as $20 \mathrm{GeV}$. Also, provisions are made 

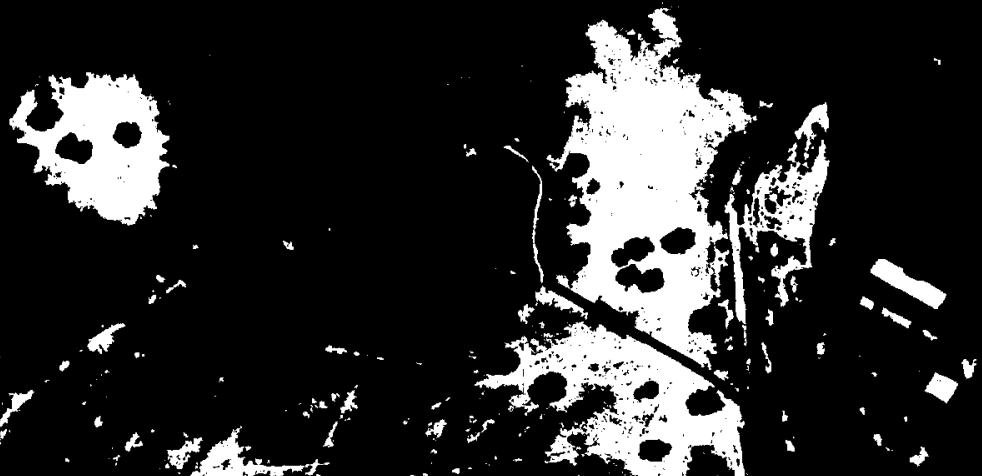

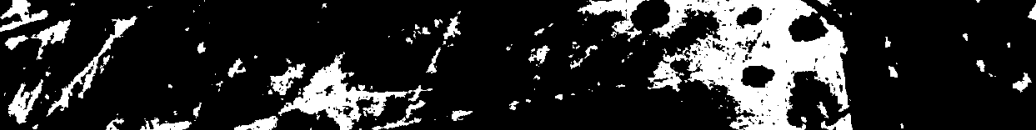

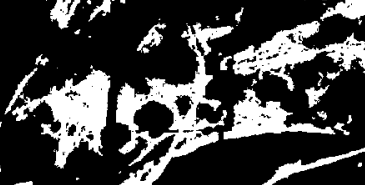

$\lim ^{2}$

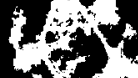

sitis

$+18$

is

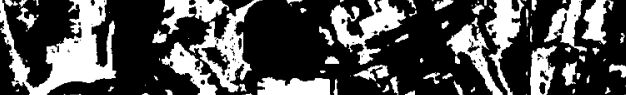

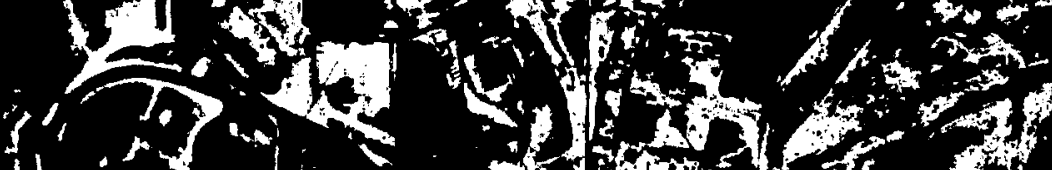

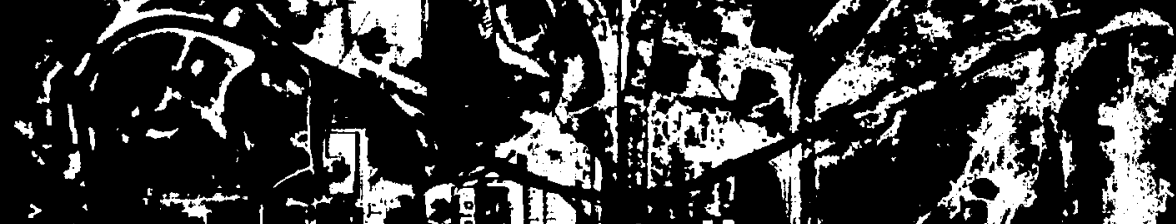

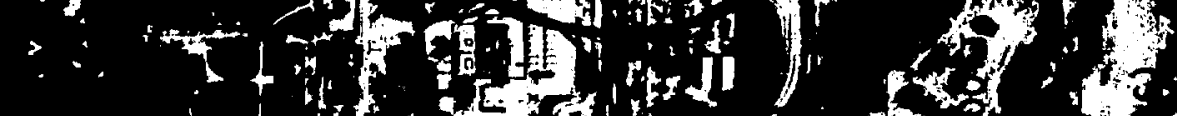

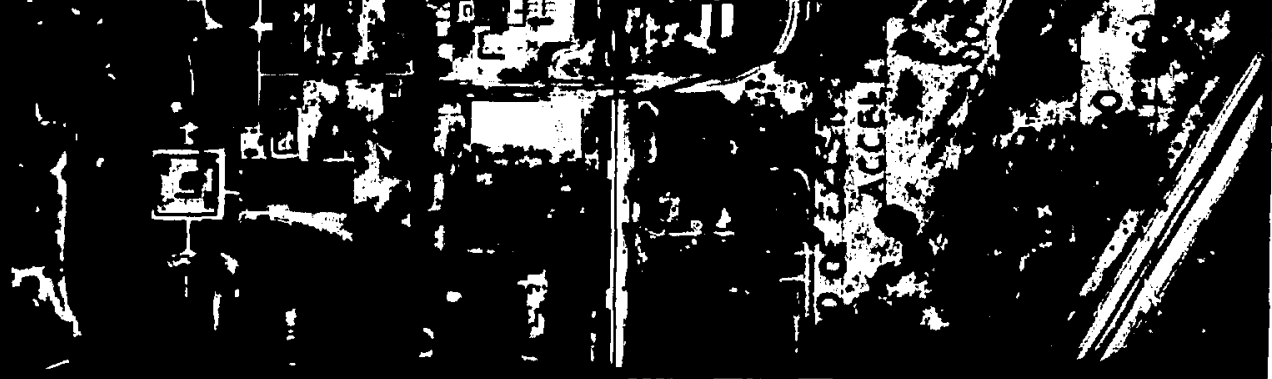


in the design of the ring huusing so that a synchrotron-radiation research facillty could be added in the future.

The energy lost from the beams by synchrotron radiation is restored by a high-power radiofrequency accelerating system which employs klystrons to drive the accelerating structure at a frequency of about $360 \mathrm{MHz}$ and which is capable of delivering several megawatts of power to the beams. Since this power appears as synchrotron radiation which strikes the outer wall of the vacuum chamber, that wall must be water-cooled. Moreover, the radiation causes the evolution of gases from the vacuum chamber surface and tinese gases must be pumped away very rapidly, becau se exceedingly low pressures (about $10^{-8}$ Torr) must be maintained in the vacuum chamber to achieve adequate beam lifetimes (several hours) and low experimental background counting rates. These low pressures will be sustained by means of long, narrow sputter-ion pumps located in the vacuum chamber in the bending magnets directly alongside the beams.

The proposed storage ring is designed to generate a luminosity (reaction rate per unit reaction cross section) of $10^{32} \mathrm{~cm}^{-2} \mathrm{sec}^{-1}$ per interaction region at a beam energy of $15 \mathrm{GeV}$. As discussed in Section II, this luminosity appears adequate to support a vigorous experimental program. To achieve this performance, it is necessary to store a current of about 100 milliamperes in each beam. Based on the expected performance of the SLAC two-mile accelerator in filling SPEAR II, the filling time for PEP will be ten to fifteen minutes which is a comfortably short period compared to the storage time of several hours, and ensures that storage ring operations will consume only a small fraction of the linear accelerator beam time.

The fundamental limitation on the performance of existing electron-positron storage rings is the so-called "incoherent beam-beam instability" which imposes 
an upper limit on the current density of the beams where they collide. The magnetic guide field of PEP is designed to attain the specified performance, as described in Section III, within the limitations established by this instability. There are other instabilities which have been observed in operating storage rings affecting both single stored beams and colliding beams, but techniques have been found to cure or to avoid them and these techniques are incorporated into the PEP design. For these reasons, the prujection of luminosity should be reasonably accurate.

Each counter-rotating beam will be concentrated into three bunches, each a few centimeters long, equally spaced around the ring, with the result that the bunches will collide at only six places. The filling process will be controlled so that the collisions will take place at the centers of the six long straight sections, called interaction regions. Five of these interaction regions will be housed in experimental halls of various designs where the high-energy-physics experiments will be carr.ed out. These designs and the rationales for them are presented in Section IV. The sixth interaction region (northwest) will be reserved for accelerator physics measurements and experiments.

\section{Other Electron-Positron Colliding-Beam Facilities}

The first definite colliding-beam reactions were observed in a small electron-positron ring operated by a joint Italian-French group in 1963 . The first colliding-beam high-energy physics experiment was completed in 1965 with the Princeton-Stanford 500-MeV storage rings at Stanford. Since that time a substantial number of colliding-beam facilities have been built. The capabilities of the principal electron-electron and electron-positron storage rings are characterized in Fig. 2 in which their maximum center-of-mass energy (available reaction energy) is plotted against their luminosity. (The question marks 


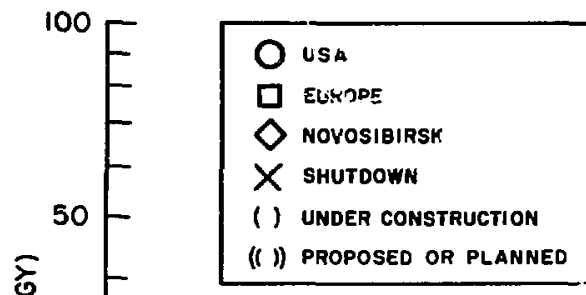

O ( $P E:$
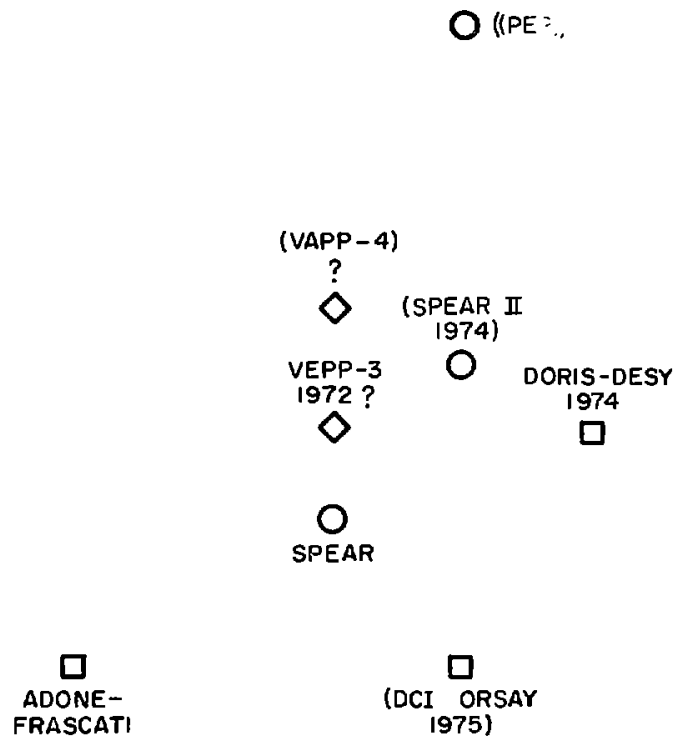

VEPP-2M

1973

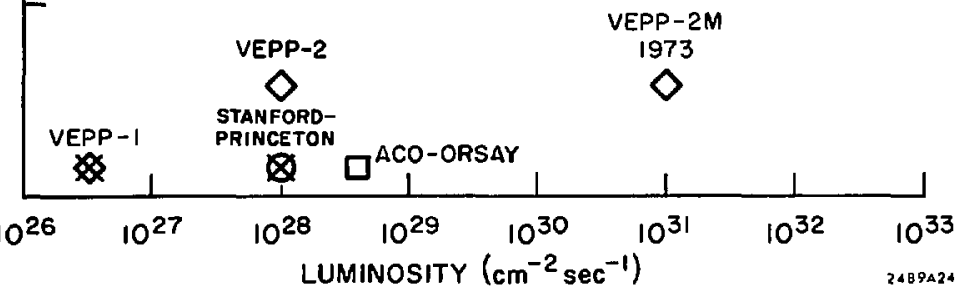

$\mathbf{Z}$

CEA

(DCI ORSAY

1975)

FIG. 2 Electron-positron colliding-beam facilities of the world. 
associated with Novosibirsk, USSR, storage rings represent our uncertainty as to when they will begin to function or when they will be built.) The early Stanford-Princeton rings and the very successful Intersecting Storage Rings at CERN in Geneva do not appear on the graph because they produce electronelectron and proton-proton collisions respectively. Design studies of electronpositron storage rings having maximum beam energies in the range $10 \mathrm{GeV}$ to $14 \mathrm{GeV}$ are under way in Italy, the United Kingdom, Germany and Japan.

\section{E. Cost Estimate and Time Schedule}

The estimated cost of the proposed storage-ring facility, expressed in Fiscal Year 1974 dollars, is $\$ 53.3$ million. Recent experience indicates that Woth labor rates and materials costs are experiencing a steady upward trend, and accordingly we have made an allowance of $\$ 8.5$ million, based on a rate of $7 \%$ per year for escalation and a four-year construction schedule. With this allowance, the grand total estimated cost of the facility is $\$ 61.8$ million. Details of the estimated costs and schedule are provided in Section $\mathrm{V}$.

The incremental annual cost of operating the facility is estimated in Fiscal Year 1974 dollars as $\$ 4$ million. 


\section{PHYSICS WITH ELECTRON-POSITRON COLLIDING BEAMS}

\section{A. Introduction}

Electron-positron storage rings have opened a new and revealing field that is still in its infancy. The results from Adone and CEA and the first results from SPEAR at total center-of-mass collision energies up to approximately 5 $\mathrm{GeV}$ have already demonstrated that the cross sections for producing hadrons are large and exhibit strikingly unexpected behavior. In particular, detailed results from the SPEAR magnetic detector do not support many of the recently developed concepts of hadron structure based on the earlier deep inelastic electron scattering experiments. The concepts of a point-like substructure and of localized currents within the nucleon and the technique of extrapolation to the light cone have been called into question and may become applicable only at higher and as yet unexplored colliding-beam regions.

With the PEP electron-positron ring, a totally new region of parameters for exploring nature will be opened up. The proposed collision energy of 30 $\mathrm{GeV}$ will lead to hadron production with a total invariant (mass) ${ }^{2}$ of $\mathrm{s}=900 \mathrm{GeV}^{2}$. This is an order of magnitude beyond the limit $\mathrm{s}=81 \mathrm{GeV}^{2}$ expected to be explored starting in 1975 by the second stage of SPEAR and by the electron-positron ring DORIS at DESY. PEP with $\mathrm{s}=900 \mathrm{GeV}^{2}$ will advance the electromagnetic frontier to comparable total collision energies now achieved with proton beams at NAL which reach $s=750 \mathrm{GeV}^{2}$ for $400-\mathrm{GeV}$ laboratory energy, thereby enabling vital comparisons to be made between interactions initiated by electromagnetic and by strong interactions.

It is not possible to predict what will be most important and what might be discovered with $\mathrm{s}=900 \mathrm{GeV}^{2}$, particularly since the results to date have been so surprising and in disagreement with theoretical expectations, however some 
important questions that PEP could probe and answer can be delineated. In the following sections we discuss processes that are expected to have reasconable interaction rates with a colliding-beam luminosity of $\sim 10^{32} \mathrm{~cm}^{-2} \mathrm{sec}^{-1}$, the design luminosity of PEP at $s=900 \mathrm{GeV}^{2}$.

We divide our discussion into four main types of processes:

Electron-positron annihflation into hadrons;

Weak-interaction effects and new-particle production;

Tests of quantum electrodynamics;

Two-photon reactions.

We complete this section with a summary of the unique features of $e^{+} e^{-}$colliding beams.

\section{B. Annihilation into Hadrons}

1. Energy Dependence of the Total $e^{+} e^{-}$Hadronic Cross Section

Annihilation of electrons and positrons to form hadrons occurs, to first order in the fine structure constant $\alpha$, through an intermediate state of a single virtual photon of (mass) ${ }^{2}=8$, See Fig. 3. Our ideas based on the observed Bjorken ${ }^{4}$ scaling behavior in deep inelastic electron processes lead to the prediction that, when the energy of the virtual photon is much larger than the masses of known particles and resonances, a simple scale-invariant behavior of the total cross section should prevail; i.e.,

$$
\sigma_{\text {tot }}\left(e^{+} \mathrm{e}^{-}-\text {hadrons }\right) \underset{s \rightarrow \infty}{\simeq} \frac{\text { const }}{s} .
$$

This is just the same energy dependence as that for $\mathrm{e}^{+} \mathrm{e}^{-}$annihilation into $\mu^{+} \mu^{-}$ and hence the ratio

$$
\mathbf{R}=\frac{\sigma_{\text {tot }}\left(\mathrm{e}^{+} \mathrm{e}^{-} \rightarrow \text { hadrons }\right)}{\sigma\left(\mathrm{e}^{+} \mathrm{e}^{-} \rightarrow \mu^{+} \mu^{-}\right)}
$$

should be asymptotically constant for large enough $\mathrm{s}$. 


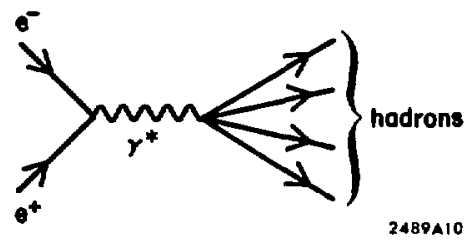

FIG. 3 Electron and positron annihilating via the one-photon $\left(\gamma^{*}\right)$ intermediate state of many hadrons. 
Quite unexpectedly the results of experiments at $\mathrm{CEA}^{5}$ and SPEAR $^{6}$ bave indicated that $\mathrm{R}$ varies with $\mathrm{s}$ in the range $9 \mathrm{GeV}^{2}<\mathrm{s}<25 \mathrm{GeV}^{2}$, where it is rising with increasing $s$. Thus the behavior of $R$ is not understood at present energies and this is particularly surprising since scaling was discovered for deep inelastic electron scattering in the same energy range.

As an indication of how significant this kind of study is to the understanding of: the structure of hadronic matter, there follows a list of fundamental questions which could be answered by measuring the cross section:

(a) Does $\mathrm{R}$ eventually become constant and if so at what onergy? Is the magnitude of $\mathbf{R}$ equal to sums of squares of charges of the nucleon's constituents as required by the quark-parton model? Is the approach to asymptotic behavior consistent with the predictions of gauge theories with a structure leading to a scaling behavior; 1.e., does $R$ approach a constant value from above as $|a|+|b| / \ln s ?^{7}$

(b) Does $R$ have one value for intermediate $s$ and another larger value for large s, indicating that one is crossing thresholds for the production of new particles with quantum numbers different from those of the known hadrons; 1.e., production of "charmed" or "colored" states? 8

(c) Does $\mathrm{R}$ have irregularities associated with the opening of new vectormeson channels which couple to the electromagnetic current? The $\rho^{\prime}$ enhancement at $\approx 1.5 \mathrm{GeV}$ is one such candidate and there may be others lying on appropriate Regge trajectories crossing $J=1$.

(d) Does $\mathrm{R}$ have an $\mathrm{s}$ dependence, suggesting that there are additional layers of substructure of the nucleon that are probed at higher energies, $s 25 \mathrm{GeV}^{2}$, before one "sees the light cone"? 
(e) Does $\mathbf{R}$ grow continuously with $\mathbf{s}$ ? If this were true up to the value of $s=900 \mathrm{GeV}^{2}$, observable deviations from the predictions of quantum electrodynamics would be introduced. 9

(f) What is the mean multiplicity of hadrons as a function of $s$ ? For example, does the present very puzzling excess of neutral secondary hadrons above predictions continue or even increase with $s$ ?

Clearly, the various features of the total hadronic cross section emerge as fundamental to an understanding of hadronic structure.

2. Inclusive Production Cross Sections with Detection of One Hadron

With $30-\mathrm{GeV}$ colliding beams it is possible to study the inclusive reaction

$$
e^{+} e^{-} \rightarrow h+\text { anything , }
$$

for large energy and transverse momentum, i.e., transverse to the beam axis, deposited on the one detected hadron $h$. If the total cross section is observed to approach the asymptotic behavior described by Eq. (1) in this kinematic region, it may be reasonable to neglect all hadronic masses or constituent masses in studying inclusive distributions so that no intrinsic dimensions remain and all cross sections exhibit a scaling behavior. It is just this scaling behavior that we derive from an elementary point-constituent picture of the nucleon. In the one-photon approximation, the scaling law takes the form ${ }^{10}$

$$
\frac{d^{2} \sigma}{d w d \cos \theta}=\frac{1}{s}\left[f_{1}(w)+\sin ^{2} \theta f_{2}(w)\right]
$$

where $\theta$ is the angle between the detected hadron and the colliding-beam axis, and $w$ is the hadron energy divided by the beam energy. It is already clear from the SPEAR results that this scaling law is not obeyed over the complete range of $\mathbf{w}$. 
Some fundamental questions which can be answered at higher energies with PEP are:

(a) Do the inclusive cross sections (Eq. (3)) scale with $1 / \mathrm{s}$ ?

(b) What are the magnitudes and $w$ dependences of $f_{1}(w)$ and $f_{2}(w)$ for different hadrons such as $\pi, K, N, \Sigma, \Lambda$, etc.? Can they be related in any way to the analogous structure functions for deep inelastic scattering of protons?

(c) How accurate are the predictions of invariance principles such as charge conjugation invariance and SU3 symmetry at high energies?

One of the most intriguing observations at SPEAR has been the similarity of the momentum distribution of secondary hadrons from $\mathrm{e}^{+} \mathrm{e}^{-}$collisions to the transverse momentum distribution of secondary hadrons in purely hadronic inclusive reactions, and in particular in p-p collisions. Whether this hadronic similarity of $\mathrm{e}^{+} \mathrm{e}^{-}$annihilation events persists at higher $\mathrm{s}$ values, and, what its underlying significance is, are important questions to be investigated with PEP.

It is of interest to contrast a scaling law such as Eq. (4) with the observed scaling behavior in the hadronic inclusive reaction $\mathrm{pp} \rightarrow \pi^{0} \mathrm{X}$ for the case of the $\pi^{0}$ emerging at $90^{\circ}$ in the center -of-mass system. In this case the data for large transverse momenta, $p_{\perp}$ up to $9 \mathrm{GeV} / \mathrm{c}$, is well fitted by a form with the much faster fall-off ${ }^{11}$ of

$$
E \frac{d^{3} v}{d p^{3}} \approx \frac{1}{p_{\perp}^{n}} f\left(\frac{p_{1}}{\sqrt{s}}\right)
$$

where $f$ is a scale-invariant function presumably reflecting the fundamental interactions at short distances. The observed value of $n$ is $n \approx 8-10$ as compared to the value $n=4$ predicted for high energies, by the scaling law Eq. (4) for inclusive $\mathrm{e}^{+} \mathrm{e}^{-}$annihilation. 
The critical parameter in the p-p inclusive reactions is not the square of the total reaction energy $s$, but rather $s_{\text {eff }}=4 p_{1}^{2}$ which is a measure, at large production angles, of the energy of the subprocess illustrated in Fig. 4. Since the electron-positron pair in PEP converts all of its collision energy into hadrons, the effective energy and the total energy are equal, and with the PEP parameters, $s=(30 \mathrm{GeV})^{2}$. In hadron collisions at the CERN ISR and at NAL, experiments have presently reached a maximum effective collision energy of $s_{\text {eff }} \approx(18 \mathrm{GeV})^{2}$. It will be of great interest to be able to compare the large-momentum secondary hadron distributions from $\mathrm{e}^{+} \mathrm{e}^{-}$annihilations with those from $\mathrm{p}-\mathrm{p}$ collisions at the higher available collision energies or equivalently at the maximum values of $p_{\perp}$.

3. Inclusive Production Cross Sections with Detection of Several Hadrons

The behavior of the cross section when many hadrons are observed in an inclusive process can also be studied at PEP; e.g., the inclusive process

$$
e^{+} e^{-} \rightarrow h_{1}+h_{2}+\cdots+h_{n}+X
$$

Just as in the case of process (3), the energy-dependence of the cross section is important, but in addition in this case, the hadron multiplicity as a function of energy as well as correlations among the detected hadrons yield further information about hadron structure. In a model where the photon decays into a pair of point-like constituents (partons) which subsequently decay into hadrons, we can expect a striking feature: the formation of jets associated with the decay of the constituent partons into the final hadrons. See Fig. 5. Since the pointlike constituents are produced at all angles, these decay jets should appear also at all angles. That the decaying hadrons should have most of their momentum along the direction of the parent constituent parton is a natural conclusion based 


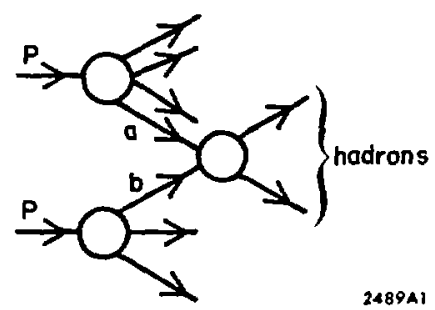

FIG. 4 Proton-proton collision with constituent particles $a$ and $b$ interacting to produce the observed hadron at large $p_{1}$. 


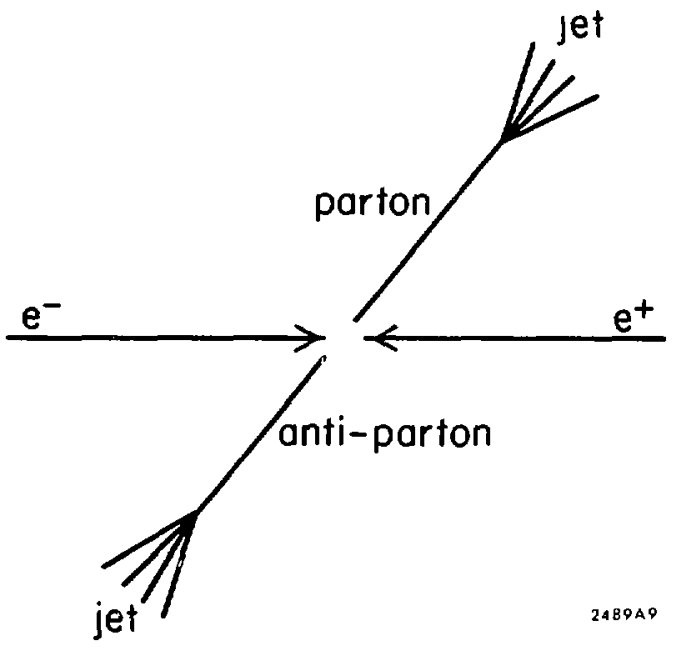

FIG. 5 Electron-positron annihilation into parton and anti-parton with the parton and antiparton decaying into the final hadron jets. 
on the fact that purely hadronic reactions are dominated by small uranswerse momentum processes. Experimental observation, both of jets at large transverse momentum and of scaling behavior, would offer a very striking confirmation of this kind of constituent model.

These examples show that studies of the inclusive processes at high energy and transverse momentum offer the possibility of a wealth of new phenomen $z$ which are at present unexplored.

\section{Weak Interactions and New Particle Production}

\section{Heavy Leptons $\left(\mu^{*}\right)$}

The theoretical interest in heavy leptons has been considerably heightened recently because of their relevance to the gauge theories of weak interactions. If point-like particles similar to the muon exist and if their mass is less than about $13 \mathrm{GeV}$, then they will be copiously produced by $\mathrm{e}^{+} \mathrm{e}^{-}$collisions at rates comparable to that of the $\mu^{+} \mu^{-}$channel. For a $\mu^{*}$ mass up to about $13 \mathrm{GeV}$, the expected counting rate is approximately

$$
\mathrm{Y}=\left(10^{-34} \mathrm{~cm}^{2}\right) \mathscr{L} \approx 10^{-2} \text { counts } / \text { sec }
$$

for a luminosity of $10^{32} \mathrm{~cm}^{-2} \mathrm{sec}^{-1}$, independent of mass. Since the $\mu^{*}$ would have a very short lifetime $\left(\tau \approx 10^{-16}\right.$ sec for $\left.M_{\mu *} \approx 10 \mathrm{GeV}\right)$, the detection of these particles would be through their decay products.

A theoretical understanding of the mass spectrum of leptons is one of the most challenging problems in elementary particle physics, and at present it is lacking. All possible experimental investigations relating to this question will be of very great importance. 


\section{Weak Intermediate-meson (W) Pairs}

It has been postulated in analogy to quantum electrodynamics that the weak interactions are mediated by massive charged vector mesons (W mesons). Experimental investigations have been performed using all presently available particle beams and energies with the hope of finding the $\mathrm{W}$. The fact that it has not yet been detected (1974) could mean that its mass is beyond the available energy threshold or that the cross section is so small that the appropriate signal is hidden in the background of more prominent channels. If there are point-like charged W mesons of mass less than $15 \mathrm{GeV}$, they would be copiously produced Ly PEP.

The electromagnetic interaction between a photon and a spin-one boson involves, in addition to the charge, the magnetic moment and the electric quadrupole moment of the boson. Assuming the charge form factor is equal to one, while the magnetic moment and quadrupole form factors are zero, the total cross section is given by ${ }^{12}$

$$
\sigma_{\overline{\mathrm{BB}}}=\frac{2.2 \times 10^{-32} \mathrm{~cm}^{2}}{\mathrm{~m}_{\mathrm{B}}^{2}}\left[1-\left(\frac{\mathrm{m}_{\mathrm{B}}}{\mathrm{E}}\right)^{2}\right]^{3 / 2}\left[1+\frac{3}{4}\left(\frac{\mathrm{m}_{\mathrm{B}}}{\mathrm{E}}\right)^{2}\right],
$$

where $m_{B}$ is the boson mass in GeV. At high energy, the total cross section becomes consiant, whereas the point-like constituent cross sections discussed earlier might decrease with increasing energy as $1 / E^{2}$. Electron-positron annihllation into boson pairs may be a very efficient means of producing these particles if they indeed exist.

\section{Neutral Current Effects}

Reactions with neutrino beams at $\mathrm{CERN}^{13}$ and $\mathrm{NAL}^{14}$ have been reported suggesting the existence of neutral weak currents coupling neutrinos to hadrons. 
Such weak neutral currents are required by many of the gauge theories ${ }^{15}$ of weak interactions which have been constructed recently with the goal of unifying weak and electromagnetic phenomena and thus removing the divergence difficulties associated with the usual Fermi theory. The salient point for $\mathrm{e}^{+} \mathrm{e}^{-} \mathrm{col}-$ liding beams is that these gauge theories also require that electrons and muons should have comparable coupling to the weak neutral current. Independent of the gauge theories, however, the general question of whether charged leptons also give rise to weak neutral current effects is of fundamental importance. It is expected that the weak current effects would manifest themselves by interference of the axial part of the weak current with the normal electromagnetic current giving rise to angular asymmerties or helicities that indicate parity violation. Some possibilities are listed below for the reaction $e^{+} e^{-} \rightarrow \mu^{+} \mu^{-}$. In such studies, we can take advantage of the predicted high transverse electron polarization in the initial beams resulting from the synchrotron radiation. ${ }^{16}$ This polarization will enhance the interference effects for certain kinematic regions.

To estimate the size of the neutral current effects, the Weinberg model of gauge theories is employed. Although the validity of this model is not established, it allows for a numerical prediction in terms of the CERN experiment. ${ }^{13}$ These estimates are to be viewed only as a qualitative estimate of the effects to be expected which turn out to be rather large for the proposed PEP parameters. The major effects in the $\mu^{+} \mu^{-}$channel are:

(a) Asymmetry in the Muon Angular Distribution. Whereas the ordinary one-photon contribution is front-back symmetric, the axial vector part of the weak neutral current gives rise to an interference term and an asymmetry of 
the form ${ }^{17}$

$\frac{\mathrm{d} \sigma(\theta)-\mathrm{d} \sigma(\pi-\theta)}{\mathrm{d} \sigma(\theta)+\mathrm{d} \sigma(\pi-\theta)}=\frac{\sqrt{2} \mathrm{Gs}}{4 \pi \alpha} \frac{\cos \theta}{\left(1+\cos ^{2} \theta-\mathrm{S}_{t} \mathrm{~S}-\sin ^{2} \theta \cos ^{2} \phi\right)}$

where $\theta$ and $\phi$ are the polar and azimuthal scattering angles, $G=10^{-5} \mathrm{M}_{\mathrm{p}}^{-2}$ is the Fermi constant and $S_{-}, S_{+}$are the electron and positron transverse polarizations (which are predicted to be approximately 0.92 ).

For $\mathrm{s}=900 \mathrm{GeV}^{2}$ the maximum effect is $17 \%$ which would be easily measurable, It increases linearly with $s$ for $s / M_{Z}^{2} \ll 1$ where $M_{Z}$ is the mass of the particle which carries the weak neutral current. This dependence should be readily experimentally separable from the asymmetry due to the interference between one- and two-photon intermediate states which is expected to be logarithmic in energy.

(b) Muon Helicity. Again the axial vector interference gives rise to a final muon helicity which is absent in the one-photon case. The predicted helicity is

$$
\lambda_{ \pm}=[ \pm(2 \mathrm{Gs}) / 8 \pi \alpha]\left(3 \sin ^{2} \theta \mathrm{W}-\cos ^{2} \theta \mathrm{W}\right)\left[1 \pm \frac{2 \cos \theta}{\left(1+\cos ^{2} \theta-S_{+} \mathrm{S}_{-} \sin ^{2} \theta \cos ^{2} \phi\right)}\right]
$$

where ${ }^{\theta} \mathrm{W}$ is the Weinberg angle which relates the relative amount of vector and axial vertor contributions and the $\mathrm{Z}$ meson mass. The central value resulting from the CERN experiment is $\sin ^{2} \theta_{W}=0.3$ which leads to about a $7 \%$ effect for the helicity maximum at $\mathrm{s}=900 \mathrm{GeV}^{2}$. Unlike the helicity, the asymmetry is independent of the Weinberg angle and therefore independent of the $\mathrm{Z}$ mass (for $\left.s / M_{Z}^{2} \ll 1\right)$. The two-photon interference term could also yield nonvanishing helicities, but again the effect is not expected to rise linearly with s. 
(c) Hadronic Effects. In addition to the effect in the $\mu^{+} \mu^{-}$channel, asymmetries can be expected in the hadronic channels due to weak-electromagnetic interferences. For example, in an inclusive reaction, there shor 'd be a linear term in $\cos \theta$ just as in the $\mu^{+} \mu^{-}$case. Explic it predictions for the asymmetry are very model dependent in this case and depend on the hadronic cross section which is not as calculable as in the $\mu^{+} \mu^{-}$case. The relative size of all these weak-electromagnetic interference effects rises linearly with s, a feature which is common to almost all weak interaction theories when $s / M_{Z}^{2} \ll 1$.

\section{Tests of Quantum Elentrodynamics (QED)}

Quantum electrodynamics is the only fully successful theory in elementary particle physics. Today there are no discrepancies between its predictions and the experiments which probe to ever smaller distances by means of measurements with higher momentum transfers and higher precision. The conceptual basis of QED is very simple, being built in successive steps by applying the ordinary rules of quantum theory to the classical electromagnetic field amplitudes which obey the Maxwell equations. Both the notions of local fields with differential wave propagation and of point interactions are taken over from the classical theory. One wonders whether this is a precise procedure or only an idealization that we can adopt in the sense of a correspondence principle when probing with low enough resolution. On the basis of experimental evidence reported so far, the theory is accurate. 18 Intermediate photon and electron propagators with virtual (mass) ${ }^{2} \approx \pm 10 \mathrm{GeV}^{2}$ are probed in experiments with an overall accuracy of about $10 \%$, leading to the conclusion that any breakdown or modification of QED will occur for (momenta) ${ }^{2}$ beyond about $100(\mathrm{GeV} / \mathrm{c})^{2}$ or on a scale of small distances corresponding to a length $L \leqslant 2 \times 10^{-15} \mathrm{~cm}$. With PEP, the limit of posslble breakdown of QED can be pushed another order of 
magnitude to about $10^{-16} \mathrm{~cm}$ in processes which probe space-like and time-like virtual photons (Fig. 6a), those which probe for differences in internal structure between the electron and muon (Fig. 6b) and those which probe virtual lepton propagators far from the mass shell (Fig. 6c).

These tests of QED are feasible with PEP. In particular, the calculated cross section for the process of Fig. $6 \mathrm{~b}$ is

$$
\sigma_{\text {tot }}\left(\mathrm{e}^{+} \mathrm{e}^{-} \rightarrow \mu^{+} \mu^{-}\right)=\frac{4 \pi \alpha^{2}}{3 s}=\frac{2 \operatorname{lnb}}{\mathrm{E}_{\mathrm{GeV}}^{2}},
$$

where $\mathrm{E}_{\mathrm{GeV}}$ is the beam energy in $\mathrm{GeV}: \mathrm{s} \equiv 4 \mathrm{E}^{2}$. At $\mathrm{s}=900 \mathrm{GeV}^{2}$ this corresponds to a counting rate of $10^{-2} / \mathrm{sec}$ for a luminosity of $10^{32} \mathrm{~cm}^{-2} \mathrm{sec}^{-1}$.

\section{E. Two-Photon Processes}

The amplitude for two-photon annihilation by the process

$$
e+e^{ \pm} \rightarrow e+\gamma^{*}+e^{ \pm}+\gamma^{*} \rightarrow e+e^{ \pm}+X
$$

is illustrated in Fig. 7. Although this process is of order $\alpha^{4}$, whereas the familiar lowest order one-photon contribution is of order $\alpha^{2}$, two factors operate to overcome this added factor of $\alpha^{2}$ and to promote the process to importance. ${ }^{19}$ First, the cross section for the one-photon process should decrease at high energies as $1 / \mathrm{s} \approx 1 / \mathrm{E}^{2}$ according to point-like-constituent theories. In contrast, the "almost real" photons radiated by the electrons favor large impact parameter collisions and the cross section will vary as $1 / M_{x}^{2}$ where $M_{x}$ is commonly the threshold mass of the state formed in the photon-photon annihilation. Second, the cross section should also be enhanced by familiar logarithmic factors of the form $\ln \left(E / M_{e}\right)$, one for each of the electron lines, and these are sizable ( $\sim 10$ for $E=15 \mathrm{GeV})$. Additional logarithms are also expected depending on the 

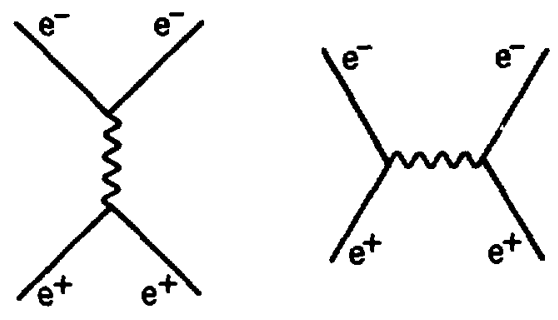

(a)

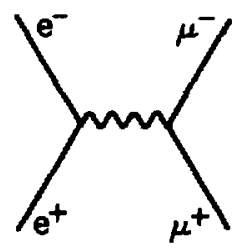

(b)

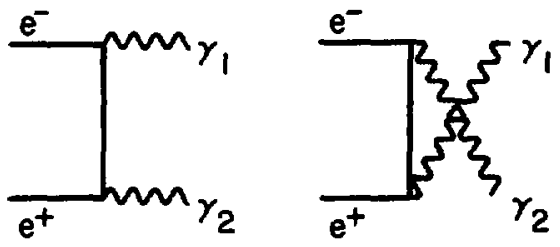

(c)

248942

FIG. 6 Quantum electrodynamical processes. 


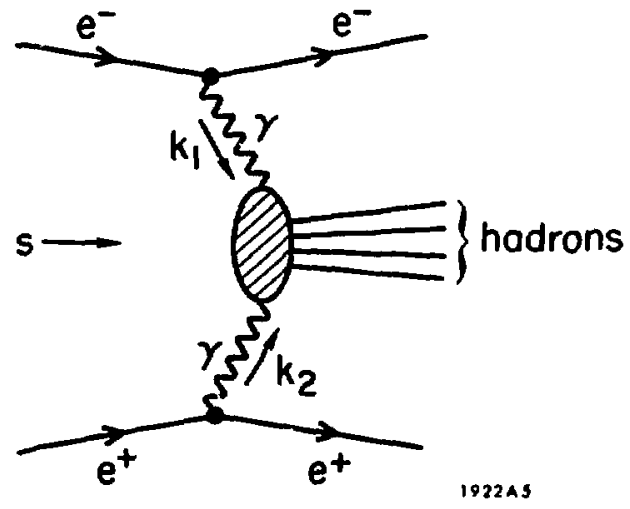

FIG. 7 Electron-positron scattering and producing hadrons via the two-photon intermediate state. 
high-energy behavior of the $\gamma-\gamma$ annihilation process. Thus $\mathrm{e}^{+} \mathrm{e}^{-}$collisions offer the possibility of studying the field of hadronic states with even $\mathrm{C}$ as produced from two photons. The cross sections are large, at least for low-mass hadronic final states. By detecting the final leptons and determining the energies $k_{1}$ and $k_{2}$ of the two colliding photons, one can measure in detail the difforential cross section for two colliding "almost real" photons to produce any observable final state.

For the dominant part of the cross section, where both leptons scatter in the near forward direction, the equivalent photons are nearly on-shell and the hadronic aspects of their interactions are expected to dominate. In this case, we have an analogue of meson-meson colliding beams in the sense that the colliding particles are bosons. In addition, however, there can be new interactions and effects specific to local two-photon interactions. Thus measurements of the tetal cross section $\sigma_{\gamma \gamma \rightarrow \text { had }}$ are certainly of comparable importance to the total photoabsorption cross section and other total hadronic cross sections. Measurements of $\gamma-\gamma$ cross sections as a function of $\mathrm{s}$ can determine the leading $\mathbf{C}=+$ hadronic states or resonances and test fundamental ideas of duality and Regge theory. Measurements of the specific channel $\gamma \gamma \rightarrow \pi \pi$ allow a unique probe of the pion Compton amplitude and can determine the $\pi-\pi$ scattering lengths and phase shifts. The behavior of this amplitude at large $s$ and $t$ is an important test of scaling laws and fixed-pole behavior predicted by quark-parton models.

If a scattered lepton in process (12) is detected at large angles then one can probe $\gamma \rightarrow$ hadron amplitudes with one or both photons off the mass shell. Such measurements can provide critical tests of the scaling and short distance behavior predicted in the quark-parton and light-cone models. 
Estimates of this process using a parton model indicate that the counting rate may be comparable to the case of the hadronic inclusive reaction, with rates of the order 1 to 25 events per hour for a luminosity of $10^{32} \mathrm{~cm}^{-2} \mathrm{sec}^{-1}$ and for total hadron energies up to $\mathrm{E}_{\mathrm{cm}} \approx 20 \mathrm{GeV}$.

\section{F. Unique Features of $\mathrm{e}^{+} \mathrm{e}^{-}$Colliding Beams}

By way of summary we collect here some important and valuable features that are unique to high-energy $\mathrm{e}^{+} \mathrm{e}^{-}$collisions.

1. In $\mathrm{e}^{+} \mathrm{e}^{-}$annihilation, hadronic matter is produced in a unique state (up to correction of the order of $\alpha$ ): angular momentum one, negative parity, zero charge and odd charge conjugation. This limitation to a single interaction channel simplifies the interpretation and analysis. The angular distribution for any single particle is broad, varying as $a+b \sin ^{2} \theta$.

2. When a particle and anti-particle annihilate, the entire beam energy is effective in producing secondary particles. In contrast, in typical $p-p$ (or $\mathrm{e}^{-} \mathrm{e}^{-}$) collisions, most of the available energy continues down the beams as scattered protons (or electrons) or beam fragments. Only in the rare p-p collision processes involving high transverse-momentum secondary particles is there a comparable sensitivity to high-energy phenomena.

3. The hadronic production by $\mathrm{e}^{+} \mathrm{e}^{-}$collisions is directly related to matrix elements of known currents. According to the light-cone argument, the structure of the fundamental underlying currents which create the hadrons is probed at distances of order $1 / \sqrt{\mathrm{s}}$. This is $7 \times 10^{-16} \mathrm{~cm}$ at the PEP energy of $30 \mathrm{GeV}$.

4. In the light-cone or scaling limit, the numerical value of the total cross is directly related to fundamental postulates and assumptions. For example, in (asymptotically free) scaling models, the ratio $\mathbf{R}$ becomes an $s$-independent 
constant and its value determines the sum of squares of the charges of the fundamental quanta of the electromagnetic current.

5. All charged particles with point-like structure up to mass $\sim 13 \mathrm{GeV}$ are produced in pairs at PEP at appreciable rates. This could include heavy leptons, perhaps recuriences of the muon and electron. Sharp breaks in energy dependence of the annihilation cross section could then be present.

6. If a new type of hadronic matter is found, e.g., "charmed" or "colored" states, then $\mathrm{e}^{+} \mathrm{e}^{-}$colliding beams will pair-produce and identify such states. Again we note that all of the beam energy goes into the production process, and there are no complications in the interpretations due to hadrons from the initial state.

7. Theoretically, there are important dynamical questions regarding the continuation from space-Iike to time-like photon interactions. The entire question of the continuation from space-like to time-like values of the inelastic and elastic form factors is only accessible by $\mathrm{e}^{+} \mathrm{e}^{-}$annihilation.

8. Electron-positron annihilation into $\mathrm{e}^{+} \mathrm{e}^{-}, \mu^{+} \mu^{-}$and $\gamma-\gamma$ provide the fundamental tests of QED at short distances.

9. If $R$, the ratio of the hadron total cross section to the $\mu$-pair cross section, continues to grow, then there must be extraordinary modifications in both magnitude and phase to the amplitudes for the quantum electrodynamic processes $\mathrm{e}^{+} \mathrm{e}^{-} \rightarrow \mathrm{e}^{+} \mathrm{e}^{-}$and $\mathrm{e}^{+} \mathrm{e}^{-} \rightarrow \mu^{+} \mu^{-}$. If $\mathrm{R}$ becomes of order $\alpha^{-1} \approx 137$, then the perturbation theory of electrodynamics breaks down and the strong inter actions become profoundly intertwined with the QED processes.

10. PEP opens to view the whole field of two-photon processes, via measurements of the production cross sections for $\mathrm{e}^{+} \mathrm{e}^{-} \rightarrow \mathrm{e}^{+} \mathrm{e}^{-}+$hadrons. 


\section{DESCRIPTION OF THE ELECTRON-POSITRON STORAGE RING}

\section{A. Magnetic Focusing System for the Storage Ring}

1. Tables of Parameters

Table 2 preser $: 2$ summary of many of the important parameters of the PEP $e^{+}-e^{-}$storage tncluding general parameters and lattice parameters, and Table 3 gives typucal beam parameters for different operating energies and configurations.

\section{Choice of General Parameters}

The primary design goals set for the storage ring in this proposal were: (1) to cover the range of beam energies from $5 \mathrm{GeV}$ up to $15 \mathrm{GeV}$ in order to provide a range of center-of-mass energies extending approximately from that expected to be available at other smaller $\mathrm{e}^{+}-\mathrm{e}^{-}$colliding-beam machines up to that avallable at the largest proton accelera' urs; (2) to maintain luminosities in the range $10^{31} \mathrm{~cm}^{-2}$ sec $^{-1}$ to $10^{32} \mathrm{~cm}^{-2} \mathrm{sec}^{-1}$ over this range in order to provide experimentally useful reaction rates with the expected cross sections;

(3) to furnish an adequate number and variety of experimental halls (interaction regions) to permit a vigorous and varied national program of experimentation and (4) co ensure compatibility of the housings and experimental halls with the possib:e future addition of a superconducting $200-\mathrm{GeV}$ proton storage ring for e-p collisions, another 15-GeV electron ring for $\mathrm{e}^{-}-\mathrm{e}^{-}$or $\mathrm{e}^{+}-\mathrm{e}^{+}$collisions, or both additional rings. These goals together with the size, shape and geological characteristics of potential locations at SLAC led us to the choice of the six-sided storage ring shown in Fig. 1 of Section I-C. With a radiofrequency power of about $5 \mathrm{MW}$ avallable to the beams and with the arrangements for controlling the cross-sectional area of the beams described below, the storage ring should 
Table 2

\section{General Parameters}

Beam Energy, E

Nominal Maximum

$\mathrm{GeV}$

Minimum

$5 \mathrm{GeV}$

Design Luminosity per Interaction Region, $\mathscr{Z}_{\max }$

At $15 \mathrm{GeV}$

Below $15 \mathrm{GeV}$

Nominal Crossing Angle, $2 \delta$

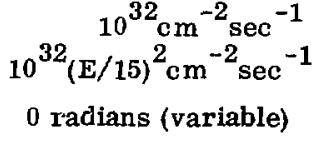

Number of Interaction Regions

Total (superperiodicity)

Available for High-Energy Physics

5

Reserved for Machine Physics Studies

1

Number of Stored Bunches, $\mathrm{N}_{\mathrm{b}}$

3

Available Length at Each Interaction Region

m

\section{Lattice Parameters}

Straight Section I,ength

$130.416 \mathrm{~m}$

Gross Radius of Arcs

$220.337 \mathrm{~m}$

Magnetic Bending Radius

$169.916 \mathrm{~m}$

Maximum Diameter of Ring

$701.505 \mathrm{~m}$

Circumference of Ring

$2166.912 \mathrm{~m}$

Cell Length

$28.842 \mathrm{~m}$

Total Number of Cells

48

Number of Standard Cells

36

Effective Length of Bending Magnets

$5.561 \mathrm{~m}$

Effective Length of Cell Quadrupoles

$0.780 \mathrm{~m}$

Bending Field at $15 \mathrm{GeV}$

$2.9447 \mathrm{kG}$

Maximum Quadrupole Field at Bore Radius

$<7.5 \quad \mathrm{kG}$ 
Table 5

Typical Beam Parameters

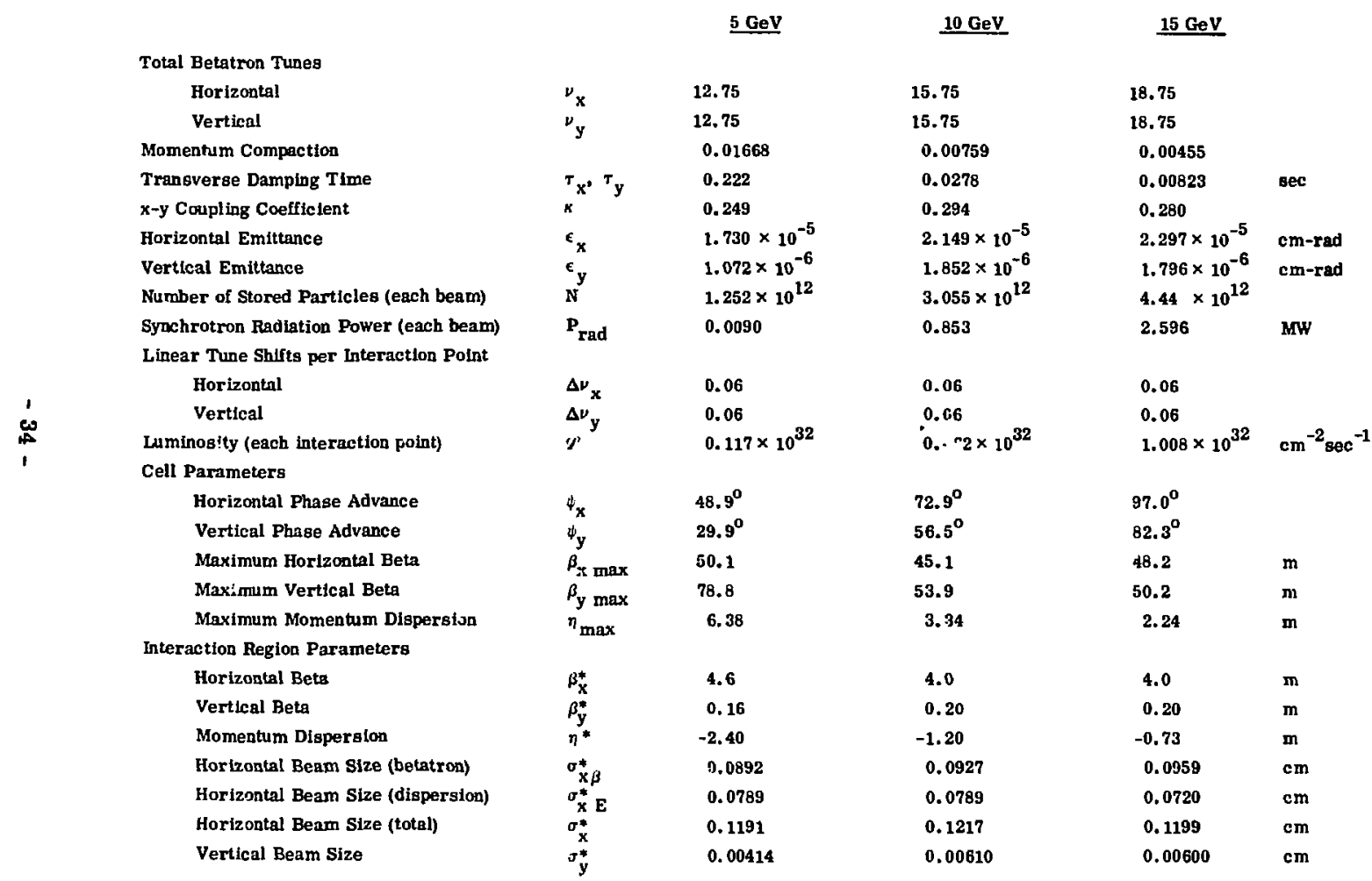


achieve a peak luminosity of $10^{32} \mathrm{~cm}^{-2} \mathrm{sec}^{-1}$ at a beam energy $E$ of $15 \mathrm{GeV}$ and a variation of luminosity approximately proportional to $\mathrm{E}^{2}$ below that energy. It may also be possible to operate the storage ring at energies somewhat higher than $15 \mathrm{GeV}$ with reduced luminosity. The design-luminosity curve is shown in Fig. 8.

The arc radius and the straight-section length are the two most influential parameters in determining the performance of the storage ring. The arc radius should be as large as possible to minimize synchrotron radiation power. Component-free drift spaces 20 meters long centered at the interaction regions have been reserved for experimental purposes. The rest of the space in the straight section is used for injection systems, if cavities and various beamcontrol elements. The straight sections are adequately long to accommodate the modifications which will be required for the proton-electron-positron option described in Appendix A.

\section{Selection of Lattice Parameters}

In order to attain high luminosity, it is necessary to collide intense beams within a small cross-sectional area. However, the number of particles which can be collided within a given area is limited by the incoherent beam-beam interaction; this limit is usually characterized by the small-amplitude vertical and horizontal tune shifts $\Delta \nu_{y}$ and $\Delta v_{x}$. It can be shown that, when beam currents are limited by the beam-beam interaction, the maximum theoretical luminosity $\mathscr{L}_{\max }$ may be increased if the beam size is enlarged. If one operates a storage ring at different energies under the same focusing configuration, the transverse beam dimensions vary directly as energy $\mathrm{E}$, and the maximum (tune-shift-limited) number of stored particles varies as $\mathrm{E}^{3}$; thus the luminosity varies as $\mathrm{E}^{4}$ and drops off very rapidly at lower energies. If, however, the 


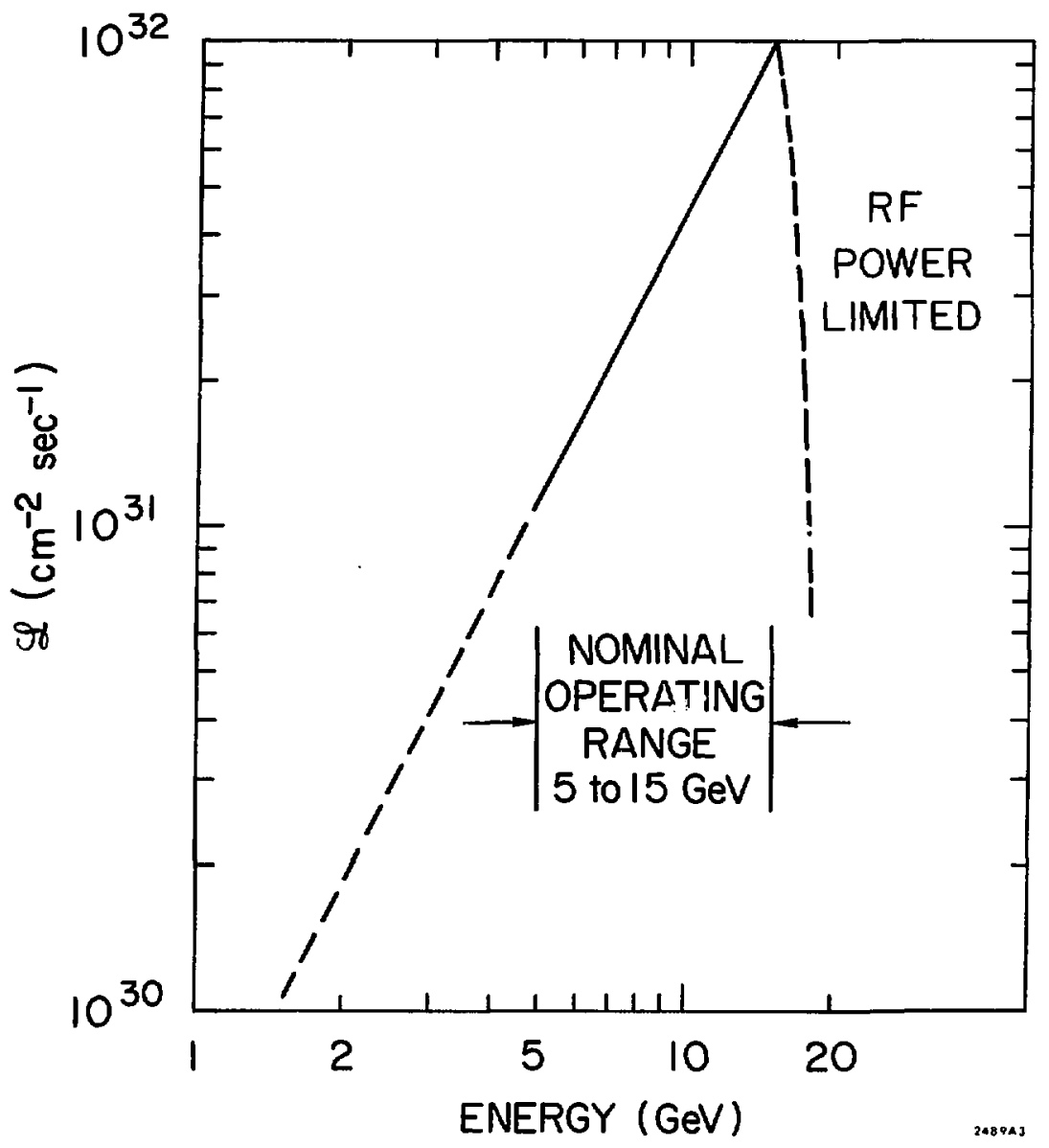

FIG. 8 Design luminosity as a function of beam enexgy showing the nominal operating range and the upper limit as limited by rf power. 
focusing configuration is changed as the energy is lowered in such a way that beam size remains essentially constant, then the maximum number of stored particles varies as $E$ and luminosity as $E^{2}$. This $E^{2}$ dependence is quite acceptable, because must reaction cross sections increase at lower energies. For energies between $15 \mathrm{GeV}$ and the maximum permitted by the magnet power supplies, the luminosity will be rf power limited and will fall precipitously.

Several different methods for beam size control will be provided. These include variation of the betatron tune, variation of the momentum dispersion function at the interaction point, and unmatching the momentum dispersion function so that it does not repeat periodically from cell to cell, but oscillates with amplitudes large compared to its match value. Vertical size will be adjusted by means of variable horizontal-vertical betatron-oscillation coupling. Using combinations of these techniques, it should be possible to reach, or at least to approach, the luminosity shown in Fig. 8.

Variation of the betatron tune gives a contribution to $\mathscr{L}_{\max }$ which varies as $\nu_{\mathrm{XA}}^{-3}$, where $\nu_{\mathrm{XA}}$ is that part of the radial tune which comes from the bending ares. Momentum dispersion gives a contribution proportional to $\eta *^{2} / \beta_{\mathrm{x}}^{*}$, where $\eta^{*}$ and $\beta_{\mathrm{x}}^{*}$ are respectively the momentum dispersion function and the betatron amplitude function at the interaction point. An unmatched dispersion function $\eta$ gives a luminosity increment proportional to $\eta_{1}^{2} / \beta_{x}^{*}$ where $\eta_{1}$ is a measure of the mismatch in the bending cells.

A lattice in which the ares consist of doublet cells and are joined by comparatively simple insertions was chosen. The structure of a cell is shown in Fig. 9 and that of an insertion is shown in Fig. 10. Preliminary studies showed that the natural beam size would be about right to give the peak design luminosity $\left(10^{32} \mathrm{~cm}^{-2} \mathrm{sec}^{-1}\right.$, at $\left.15 \mathrm{GeV}\right)$ if the bending part of the lattice contained 


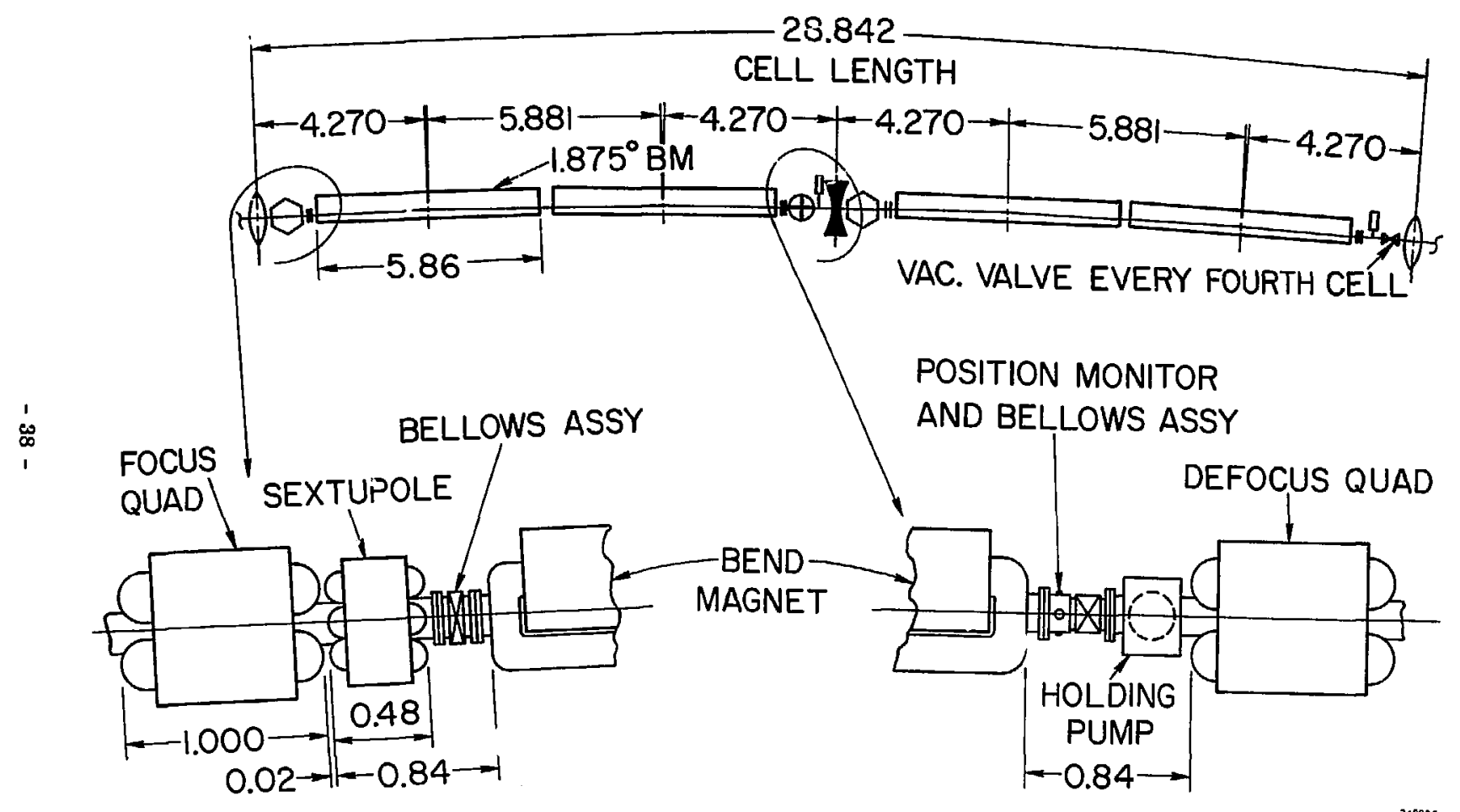

FIG. 9 Component layout of a standard cell. Dimensions are in meters. 


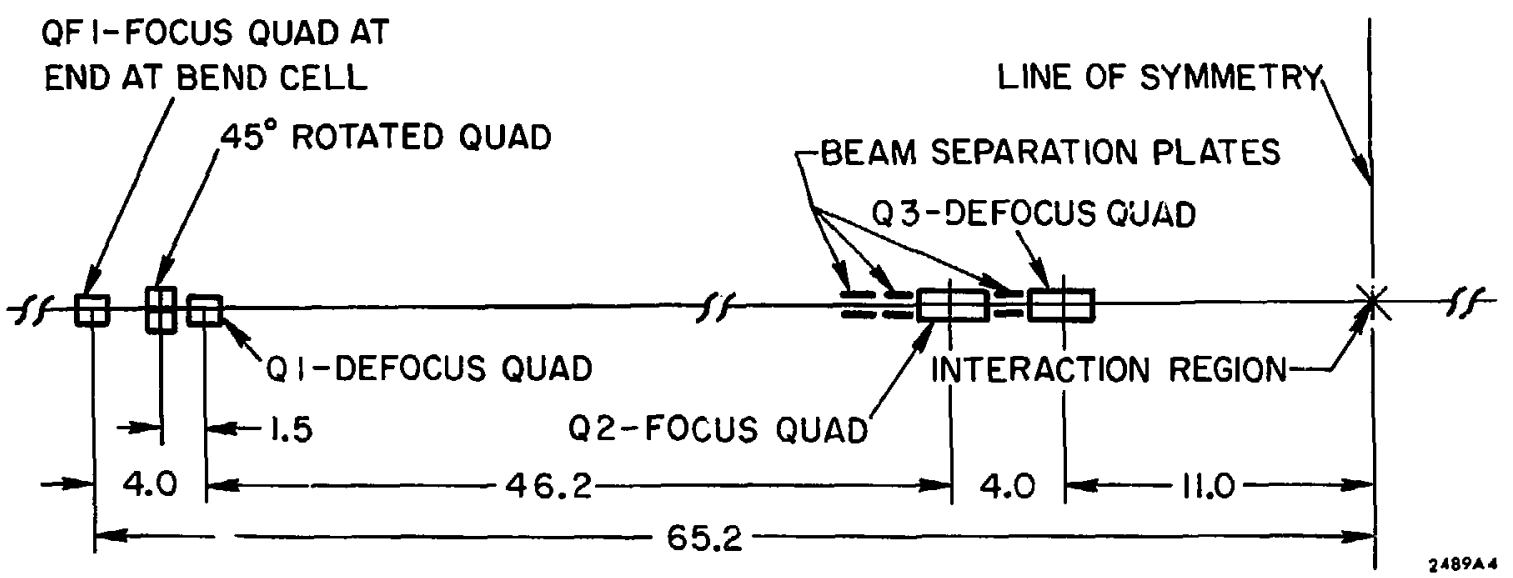

FIG. 10 The straight insertion, which is symmetric about the interaction point, is shown from the centerline of the cell quadrupole to the interaction point. Dimensions are in meters. 
between 40 and 50 cells operating with a betatron phase advance per cell of around $90^{\circ}$ in both the horizontal and vertical planes. For convenience, the number of cells was chosen to be 48 , or eight cells per 60 -degree arc. The nominal phase advance of $90^{\circ}$ per cell allows considerable latitude in varying the tune, since doublet cells work reasonably well at phase advances from below $45^{\circ}$ to above $135^{\circ}$.

The lattice requirements that have been discussed so far imply that the values of $\beta_{\mathrm{x}}^{*}, \beta_{\mathrm{y}}^{*}, \eta^{*}$ and the radial tune $\nu_{\mathrm{x}}$ should be independently adjustable. In addition, the vertical tune $\nu_{y}$ should be adjustable in order to maintain stable beam confinement. Symmetry of the ring requires that the slopes of the betatron and dispersion functions vanish at the interaction region; i.e., $\beta_{\mathrm{X}}^{\prime *}=\beta_{\mathrm{y}}^{\prime *}=\eta^{\prime *}=0$. Thus, eight mathematical constraints are imposed on the focusing strengths, requiring at least eight independently variable sets of quadrupoles. A number of other important constraints must also be considered. Tweaty meters of drift space must be kept free for experiments at the interactiun regions. The maximum values of the $\beta$-functions and $\eta$-function must be kept everywhere within reasonable bounds in order to $\min :$ nize aperture requirements and to renuce chromaticity and other aberrations. Magnetic field values must be kept within conservative limits. Furthermore, space must be reserved for various components such as if cavities, injection components (septum magnets and $k$ ickers), rotated quadrupoles, sextupoles, beam monitors and control devices, electrostatic plates for separating the beams and adjusting crossing angles and other miscellaneous instrumentation. The rf and injection systems are discussed in later sections and some of the other beam control devices are discissed later in the present section. 
A basically rather simple lattice design has evolved from the variou $\approx$ requirements mentioned above. A conventional separated-function bending cell, shown in Fig. 9, provides independent control of the total betatron tunes $\nu_{\mathrm{X}}$ and $\nu_{\mathrm{y}}$ by means of the independently controllable focusing and defocusing quadrupoles (QF and QD). The spaces between the quadrupoles and bending magnets provide room for various devices including the sextupoles, which are necessary to control chromaticity.

The insertion consists of a straight section, shown in Fig. 10, of approximately $130 \mathrm{~m}$ in length, and two modified bending cells which have standard dimensions but independently-powered quadrupoles. The four independent quadrupoles (Q3, Q2, Q1 and QF1) in the straight sections plus the two independent quadrupoles (QD1 and QF2) in the modified cells provide the minimum number of variables to match specified values of $\beta_{x}^{*}, \beta_{x}^{t *}, \beta_{y}^{*}, \beta_{y}^{\prime *}, \eta^{*}$ and $\eta^{\prime *}$ into the standard cells. The magneto-optic design programs MAGIC and TRANSPORT have been used in fitting the quadrupole gradients to the various constraints. Suitable sol:'tions have been foun! over a considerable range of values of tunes, $\beta_{\mathrm{x}}^{*}, \beta_{\mathrm{y}}^{*}, \eta^{*}$ and $\eta_{1}$ (the $\eta$-mismatch amplitude). These solutions include ranges for the various beam-enlargement schemes which are adequate to produce the design luminosity over the designated operating range of 5 to $15 \mathrm{GeV}$. Solutions which are favorable for injection also have been found.

\section{Apertures}

The "beam-stay-clear", or minimum unobstructed lateral region around the design orbit has been defined by the formula

$$
a_{i}=20 \sigma_{i}+2 \mathrm{~cm}
$$


where $a_{i}$ is the vertical or horizontal aperture diameter and $\sigma_{i}$ is the vertical or horizontal rms beam radius. The subseript denotes the particular location on the circumference. The factor of $\mathbf{2 0}$ allows sufficient, but not overly conservative, clearance to give good beam lifetimes according to experience at SPEAR; the additional $2 \mathrm{~cm}$ is a margin for orbit distortions and misalignments. The actual bore clearance of the magnets must include an additional allowance for vacuum chamber walls installation tolerances and for a possible bake-out mantle.

\section{Magnet Design}

The magnet system consists of separate bending and focusing magnets. Each main ring sextant contains 8 cells of which 6 are standard, containing 4 bend magnets, 2 quadrupoles and 2 sextupoles. The remaining 2 cells in a sextant are similar to the standard cells, but their quadrupoles operate at different levels. Figures 9 and 10 show the locativis of the various magnets in the ring and insertion areas.

The magnet system was optimized by minimizing the installed cost plus 10years' operating cost at $15 \mathrm{GeV}$. Prudent attention was given to reducing energy consumption. The syatem is capable, nevertheless, of operation at $20 \mathrm{GeV}$. Laminated magnets were selected for the main ring elements in order to minimize capital costs and to ensure sufficient uniformity magnet-to-magnet.

Two mechanical designs were considered for the bending magnets: a window-frame design and a "Cee". The "Cee" was selected because of the desirability of having an easily-accessible vacuum system. The magnet is constructed of 16-gauge low-carbon steel laminations held together by steel angles welded to the laminations. Figure 11 shows the proposed bend magnet design. 


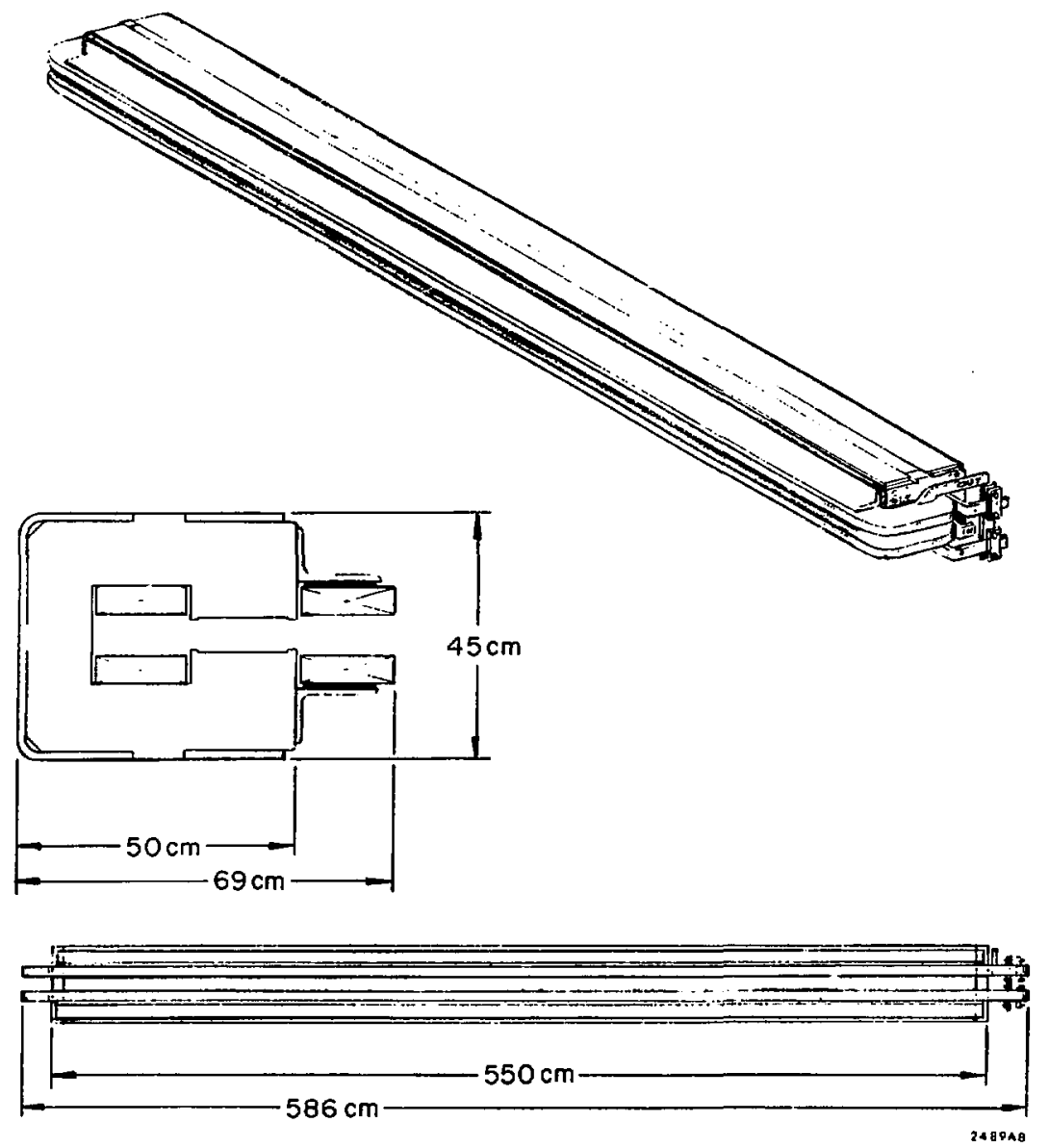

FIG. 11 Bending magnet for the electron-positron storage ring. 
In an effort to reduce costs, quadrupoles of the same aperture and lateral profile were used wherever possible. Two different apertures will be sufficient for all the guadrupoles. Variations in length will be accomplished by varying the number of laminations stacked for a particular quadrupole. Figures 12 and 13 show cross sections of the two basic quadrupole designs. The larger of the two, shown in Fig. 13, will be used for the insertion region quadrupoles Q2 and Q3. The smaller $127-\mathrm{mm}$ bore is used for all the other quadrupole magnets.

The sextupoles, unlike the other main ring magnets, will not be laminated but will be cast. The dimensional tolerances on these magnets are loose, and experience with SPEAR has demonstrated that casting is an economical way of constructing adequate sextupoles.

Small dipoles will be provided to accomplish vertical and horizontal steering. Small rotated quadrupoles will also be provided for horizontal-vertical betatron-oscillation coupling.

\section{G. Transverse Beam Instabilities}

In the design of this storage ring it has been assumed that the maximum luminosity obtainable is determined by the beam-beam incoherent limit and that it is possible to store beams of sufficient intensity to reach this limit. The various transverse instabilities that can occur with large bunched beams and might jeopardize the storage of such beams are generally thought to be of three types: those that are determined by the average circulating current, those that are present because there are multiple buncnes in the ring and those that depend upon the peak current in a single bunch.

The present PEP design appears to be rather conservative in requiring an average single--beam current of $100 \mathrm{~mA}$ in three bunches. Many electron storage rings have stored such currents at much lo:, er eneuties where the transverse 

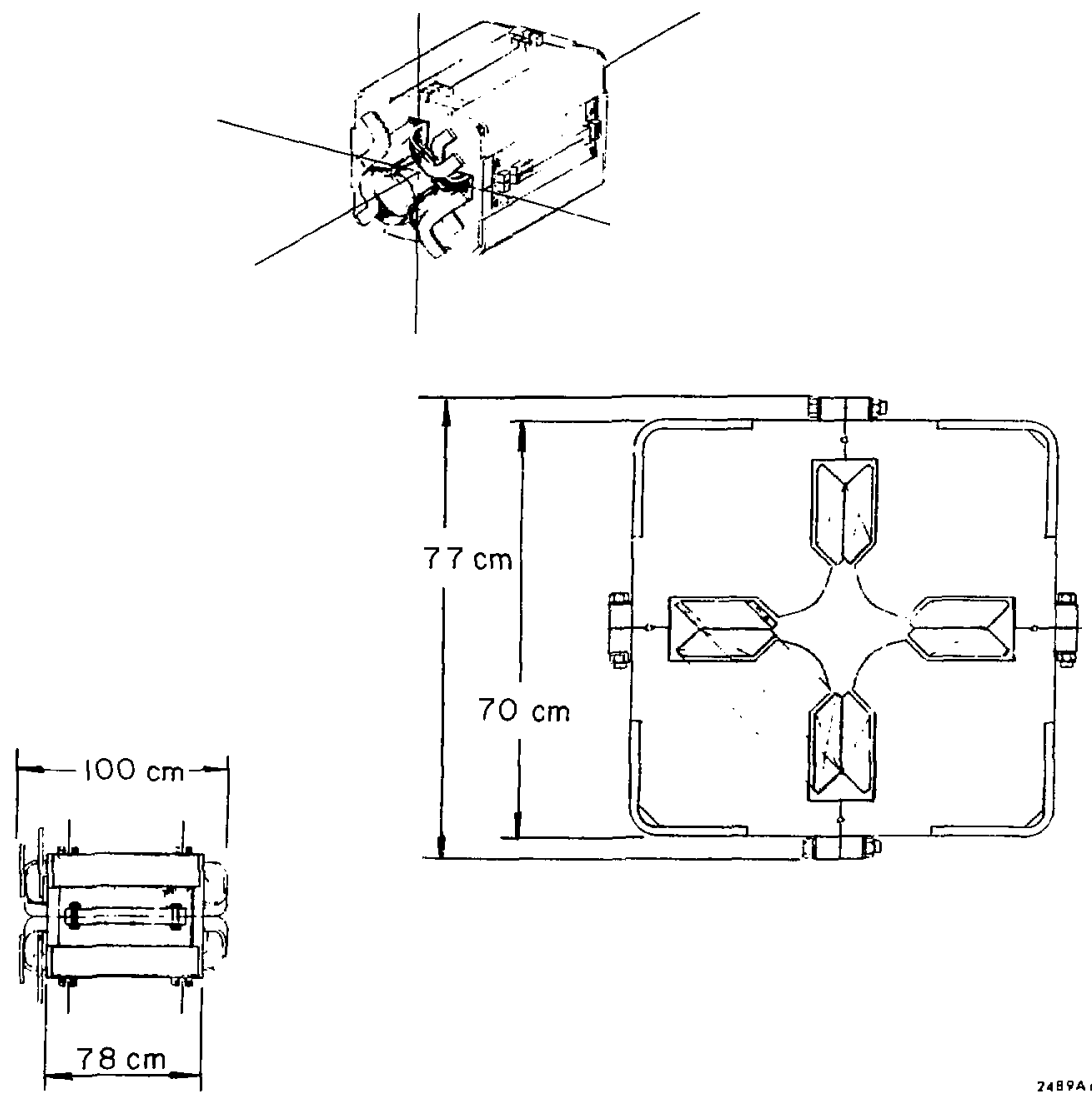

2489A

FIG. 12 Standard 127-mm-bore quadrupole used in the bending cells. 


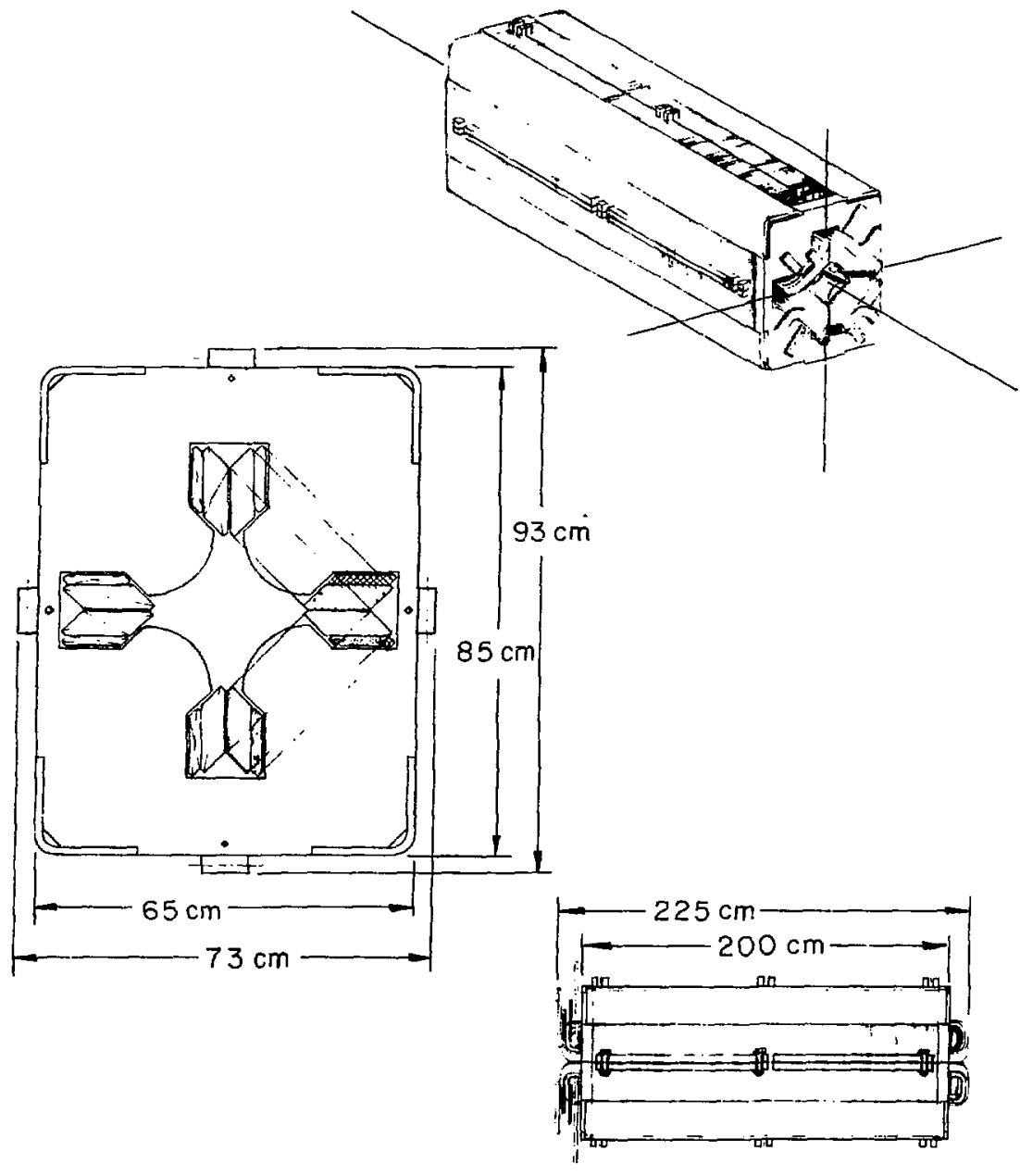

248947

FIG. 13 Large-bore quadrupole used near the interaction regions of the insertions. 
instabilities are generally much more troublesome. While multiple-bunch transverse instabilities have been predicted, they have not been observed in rings having only a few bunches and have actually been a problem only for the CEA ring. In SPEAR, in spite of careful study, it has not been possible to find a transverse multibunch instability for single beams, even with currents of over $200 \mathrm{~mA}$ per bunch. Also, because the bunch spacing in PEP is so large and the revolution frequency fixed, it is a simple matter to build a feedback system to damp each bunch individually. The only multiple-bunch motion that has been observed in SPEAR is the coherent motion between two colliding beams. This coupled motion does not seem to be of major concern in SPEAR and its effect can be reduced by use of the electric quadrupoles planned for the PEP lattice.

The head-tail instability has been the most important single-beam transverse instability observed in electron storage rings and it has been controlled by the appropriate choice of the chromaticity. In the PEP design it will be possible to vary the value of the chromaticity by means of the sextupole fields which are distributed throughout the lattice without producing destructive nonlinear resonances. The PEP design may be considered conservative in that it is presently possible to store single-bunch currents in SPEAR that exceed those necessary to reach the maximum luminosity in PEP as determined from the beam-beam limit.

\section{B. Rf System}

\section{General Af System Parameters}

The energy radiated per turn by a $15-\mathrm{GeV}$ electron circulating in the PEP ring described in Section II $\mathrm{A}$ is $26 \mathrm{MeV}$. In order to achieve a reasonable quantum lifetime, an overvoltage which depends on the lattice parameters and If frequency is required. At a frequency of $358 \mathrm{MHz}$ (chosen for reasons to be 
discussed in the following section), a peak $\mathrm{rf}$ voltage of $44 \mathrm{MV}$ is sufficient. In order to reach the design luminosity of $10^{32} \mathrm{~cm}^{-2} \mathrm{sec}^{-1}$, the required circulating current is $100 \mathrm{~mA}$ for this same lattice. The radiated power is therefore 2.6 MW per beam, or 5.2 MW total. In addition, another $2.0 \mathrm{MW}$ is dissipated in the $\mathrm{rf}$ structure.

It is proposed that the required rf power of $7.2 \mathrm{MW}$ be supplied by 24 klystrons, each delivering a CW output power of $300 \mathrm{~kW}$. Each klystron will feed an accelerator section $2.1 \mathrm{~m}$ in length, comprising five cavities. The accelerator sections will be arranged in two groups of 12 sections each, located at the ends of the southern-most straight section, shown in Fig. 1. Allowing space between sections for flanges, bellows, etc., the overall length of each group will be about $30 \mathrm{~m}$. Rf power will be supplied to the cavities through waveguldes running down vertical penetrations from the klystrons which will be housed above ground for ease of maintenance. A list of the principai parameters for the $\mathrm{rf}$ system is given in Table 4.

\section{Chcice of Frequency}

Present $\mathrm{e}^{+} \mathrm{e}^{-}$storage-ring rf systems operate at frequencies below 100 $\mathrm{MHz}$; the SPEAR If system, for exampie operates at a frequency of $51 \mathrm{MHz}$. Although operation at this low a frequency has some important advantages the shunt impedance per unit length of the cavities is only of the order of $1 \mathrm{M} \Omega / \mathrm{m}$. Thus, in order to attain the high peak voltage required for PEP using such cavities, the length of the rf structure would need to be several hundred meters. By using a higher frequency, the geometric shape of the cavities can be optimized and the shunt impedance per unit length can be increased dramatically. On the other hand, as the operating frequency is increased, the overvoltage ratio (peak voltage divided by the synchrotron radiation loss per turn) required 


\section{Table 4}

Ff System Parameters

Frequency

Energy Loss per Turn

Peak Rf Vo:'age 1

Particles per Beam

Circulating Current per Beam

Synchrotron Radiation Power (Total)

Total Accelerating Structure Length

Active Accelerating Structure Length

Total Shunt Impedance 2

Total Cavity Power Dissipation

Total Ri Power

Conversion Efficiency at Design Current ${ }^{3}$

Nuniber of $300-\mathrm{kW}$ Klystrons

Total Power Input to Rf Power Supplies ${ }^{4}$
$358.6 \mathrm{MHz}$

$26 \mathrm{MeV}$

44 MV

$4.4 \times 10^{12}$

$100 \mathrm{~mA}$

5.2 MW

$60 \mathrm{~m}$

$50 \mathrm{~m}$

$950 \mathrm{M} \Omega$

2.0 MW

7.2 MW

$70 \%$

24

11 MW

1. For a quantum lifetime of $\mathbf{1 2}$ hours.

2. The def inition of shunt impedance used in this report is $R_{s}=v_{p}^{2} / \mathbf{p}_{c}$, where $R_{s}$ is shunt impedance, $V_{p}$ is peak $r f$ voltage and $P_{c}$ is power dissipated in the cavity walls.

3. Ratio of radiated power to total $\mathbf{r f}$ power.

4. Based on klystron efficiency of $70 \%$ and a power supply efficiency of $95 \%$. 
to give a reasonable quantum lifetime also increases. Taking these two competing factors into account, it can be shown that there is a rather broad optimum for PEP in the frequency region 100 to $400 \mathrm{MHz}$.

Within this frequency region, economic and engineering considerations dominate the choice of $\mathrm{rf}$ frequency. The structure diameter, weight and cost become unreasonably large below about $200 \mathrm{MHz}$. The availability of suitable rf power sources must also be considered. A careful study of the comparative advantages of klystrons vs. gridded tubes was made in connection with the design of the rf system for the SPEAR improvement project, ${ }^{20}$ SPEAR II, and it was concluded that klystrons were superior to tetrodes with respect to initial and annual operating costs, reliability and expected life. Klystron size and cost are lowest at the upper end of the 100-400 $\mathrm{MHz}$ frequency range. This factor, togetber with the decrease in structure costs with increasing frequency, led to a choice of $358 \mathrm{MHz}$ for the SPEAR II rf system. Similar reasoning applies to the PEP rf system, and it is therefore reasonable to consider the SPEAR II system as a model for PEP. Before PEP is constructed, operational experience with the new SPEAR II rf system will have served as a test of the proposed $358 \mathrm{MHz}$ rf system design.

\section{System Design}

The requirement of $\mathrm{CW}$ operation at high energy gain, together with the need to minimize the space occupied by the rf system of a storage ring, demands an rf structure with high shunt impedance per unit length. Thus, many of the same considerations entering into the design of the shaped-cavity LAMPF accelerating structure ${ }^{21}$ also apply to the PEP structure, but there are in addicton some special design considerations. These include the large aperture required to accommodate the beam for long storage lifetimes, the need for uning 
to compensate for reactive loading and for thermal detuning effects, and the requirement to mask against intense synchrotron radiation. In addition the $\mathrm{rf}$ power transferred to the beam per unit length of structure is typically high $(\sim 100 \mathrm{~kW} / \mathrm{m})$, and the length of structure supplied by each power source is, as a consequence, limited. Taking these factors into consideration, the accelerator section which has been chosen consists of five strongly-coupled cavities operating in the $\pi$ mode so as to accelerate hoth counter-rotating beams; it is depicted in Fig. 14. Each section is driven at its center cavity by a single klystron.

The shape of the individual cavities has been optimized to produce the maximum shunt impedance which, for a perfect copper surface, would be $30 \mathrm{M} \Omega / \mathrm{m}$. The coupling slots reduce this figure by about $10 \%$. In addition, an aluminum alloy having a surface conductivity $75 \%$ that of copper is being used in the fabrication of the SPEAR $\Pi$ cavities and may also be used for the PEP structure. Taking into account the fact that the surface conductivity for real surfaces falls 5 to $10 \%$ below theoretical predictions, a shunt impedance per unit length on the order of $19 \mathrm{M} \Omega / \mathrm{m}$ can be expected for the PEP accelerating structure. The shunt impedance per unit length for the prototype cavity for the SPEAR II $\mathrm{rf}$ system was measured to be $16.5 \mathrm{M} \Omega / \mathrm{m}$. Improvements in design and in fabrication techniques should increase this figure close to about $19 \mathrm{M} \Omega / \mathrm{m}$ for the PEP structure.

The unloaded $Q$ of the structure will be about 28,000 . At maximumi operating energy where the maximum beam current of about $200 \mathrm{~mA}$ (both beams) will be stored, the beams will extract more power from the rf source than will the cavity walls, and so the accelerating units will be over-coupled with a coupling coefficient of 3.0. The corresponding loaded $Q$ is 7,000 and the filling time is 


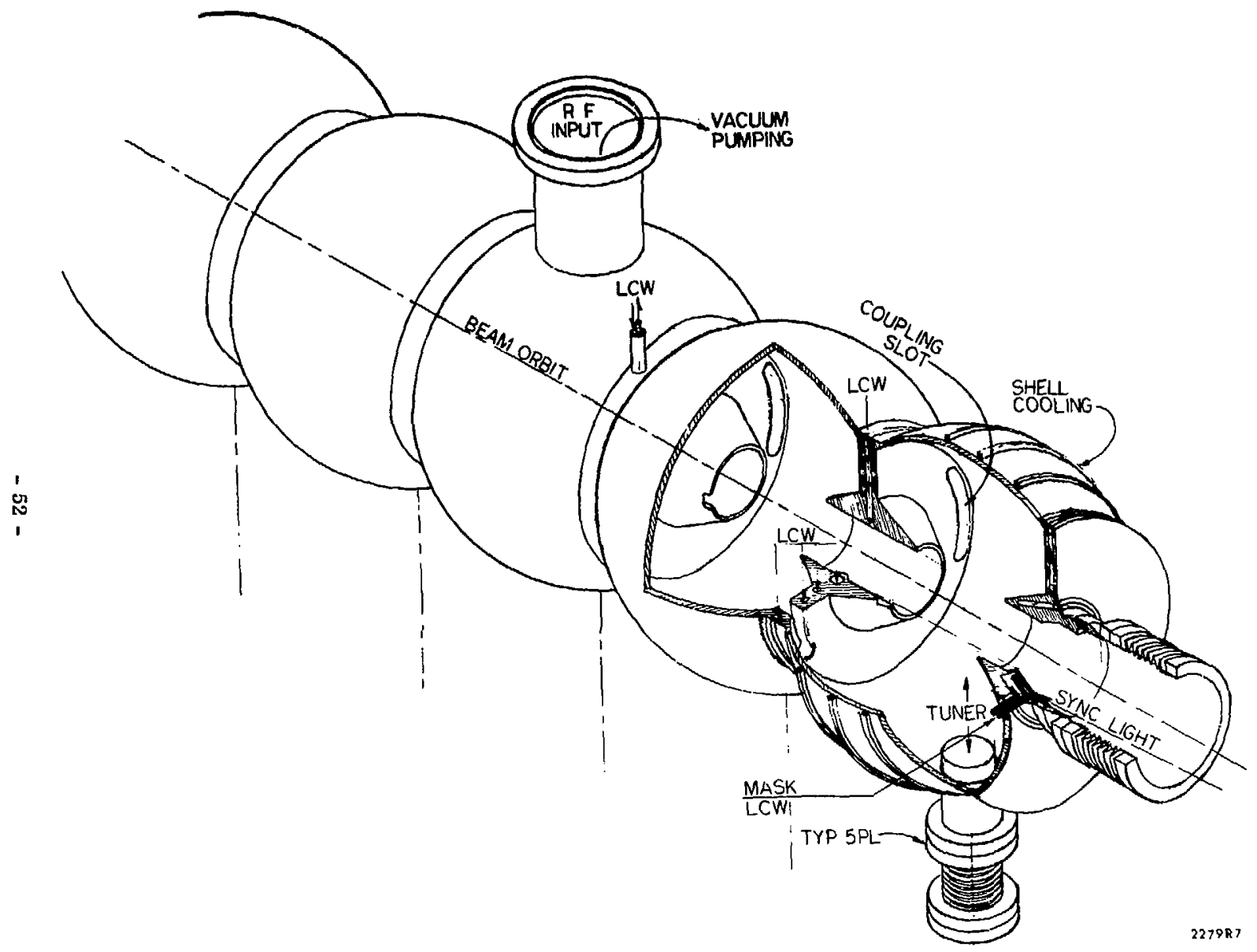

FIG. 14 Five-cavity $\pi$-mode accelerating structure used for the SPEAR II rf system and proposed for PEP. 
$6.2 \mu \mathrm{sec}$. The peak voltage which would be induced by the beam if all cavities were tuned to resonance would be $44 \mathrm{MV}$. Each cell will be provided with a tuner. These tuners will be hand-adjusted to compensate for manufacturing tolerances, and in operation they will be ganged and motor-driven to compensate for reactive beam loading and to provide a stabilizing influence on barycentric longitudinal oscillations.

\section{Klystrons}

The need for klystrons capable of supplying $300 \mathrm{~kW}$ of $\mathrm{rf}$ power $\mathrm{CW}$ at 358.5 MHz can be met with several designs. The easiest would be simply to scale the SPEAR II klystrons which have demonstrated an efficiency slightly greater than 55\%. Under these conditions, the input power to each klystron would be $550 \mathrm{~kW}$. However, in view of the present and expected future scarcity and cost of power it is highly desirable to minimize the total power consumption for the PEP If system by undertaking the development of a very-highefficiency klystron for this use. Preliminary calculations and review of klystron theory indicate that one should be able to achieve an efficiency of somewhat greater than $70 \%$ for these tubes. Under these conditions, the tube input power would be $425 \mathrm{~kW}$ instead of $550 \mathrm{~kW}$ for a total savings of nearly $3 \mathrm{MW}$ of installed capacity for 24 stations. The high efficiency will be achieved by introducing harmonic cavities and harmonic bunching. This will require additional interaction length, resulting in a klystron which is about four meters long. 
The typics 1 parameters of such a klystron operating into a matched load are:

$\begin{array}{lrl}\text { Beam Voltage } & 50 & \mathrm{kV} \\ \text { Beam Current } & 8.5 \mathrm{~A} \\ \text { Beam Power (input) } & 425 & \mathrm{~kW} \\ \text { Frequency } & 358 & \mathrm{MHz} \\ \text { Drive Power } & 10 & \mathrm{~W} \\ \text { Output Power } & 300 & \mathrm{~kW} \\ \text { Electromagnet Focusing } & 100 & \mathrm{~V} \text { at 20 A }\end{array}$

\section{Power Supplies}

The power supplies which supply beam voltage and current to the klystrons will be unregulated in the interest of economy and simplicity. The total of 24 supplies will be divided into four groups of six each. A tap changer will provide a variable ac voltage to all the supplies in each group of six as shown in Fig. 15. The voltage range will be $\pm 18 \%$ in $2 \%$ steps. The voltages from the six accelerating sections fed by the klystrons in each group will be summed, and, by means of a feedback system to the tap changer, this sum voltage will be kept constant to within the resolution provided by the tap changer steps. Fine control of the sum voltage will be obtained by varying the klystron drive.

Since all klystrons in a group of six will not necessarily operate at the same voltage, taps will be provided on the primaries of the high-voltage transformer for the klystron power supplies so that the optimum operating voltage for each klystron can be selected. Each klystron power supply will have a maximum output of $450 \mathrm{~kW}$ at $52 \mathrm{kV}$ and $8.7 \mathrm{~A}$. The supplies will be oil-filled urits with a solid-state rectifier and a 6-henry filter choke incorporated in the same oil tank. The supplies will be in weatherproofed housings located outdoors in order 


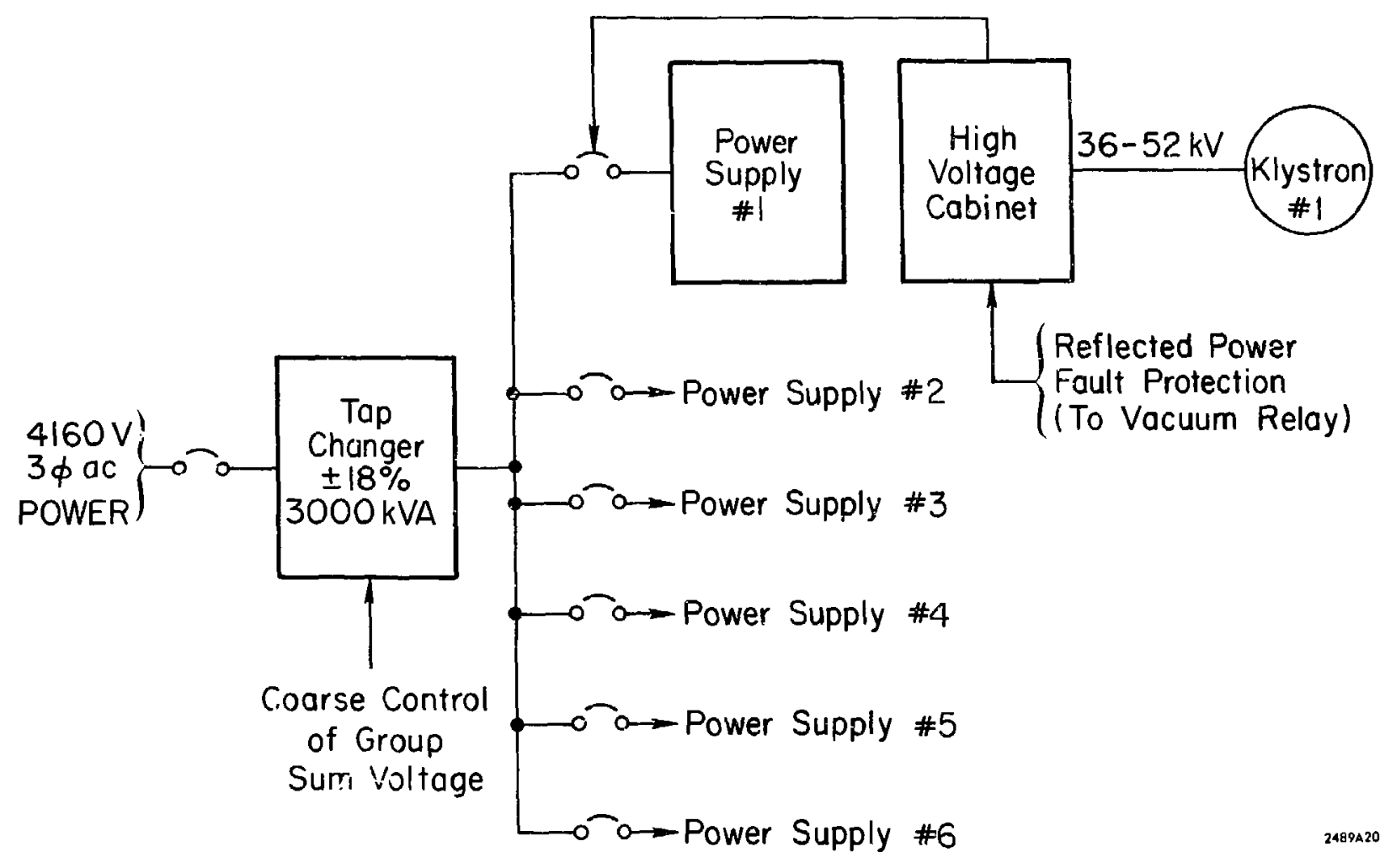

FIG. 15 Power supply arrangement for a typical klystron group showing the voltage control system. 
to minimize the fire hazard and reduce the size of the klystron building. The output of each supply will pass through the building wall to a high-voltage cabinet located just inside. The high-voltage cabinet will house a one-microfarad filter capacitor, fast shut-off vacuum relay, interlock circuits, control circuits, and voltage and current metering circuits.

In the event of a klystron fault, the energy dissipated in the fault will be minimized to $10-20$ joules by a resistor of 20 ohms and the vacuum relay, which opens in 10 milliseconds, located between the filter capacitor and the klystron. Also, in the event of a klystron arc the circuit breaker feeding its supply will be tripped off by means of a shunt trip coil.

\section{Phasing and Tuning}

A block diagram showing the drive, phasing and cavity tuning systems for the rf system proposed for PEP is given in Fig. 16. Consider first the control system for cavj:y tuning. In order to achieve optimum transfer of power to the beam, the cavity tuning must be adjusted with increasing beam current to keep the impedance real as seen by the klystron. This is accomplished by a phase bridge which compares the phase of the incidest klystron power and the phase of the field in the cavity. The output of the phase bridge activates the tiner drive which moves the tuners in or out as required to null the signal from the phase bridge.

Relative phasing of the accelerating units will be carried out by first capturing a low-energy beam with a single rf cavity on. A second cavity will be turned on and phased by maximizing the synchrotron oscillation frequency. This cavity will then be turned off and a third cavity phased to the first in a similar manner, and so on until all the cavities have been successively phased. After phasing; the largest changes tending to degrade the phase alignment will be 


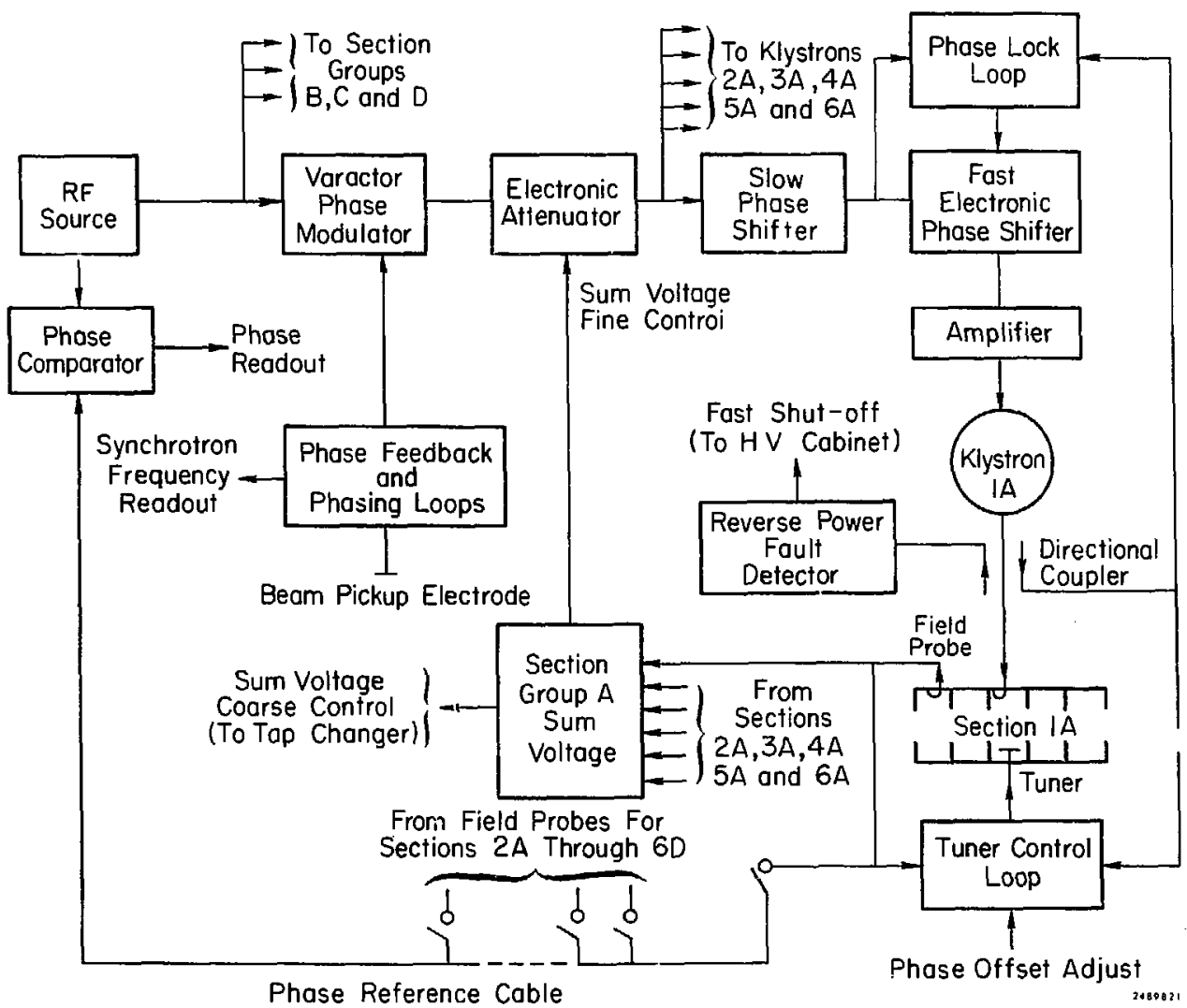

FIG. 16 Block diagram for the drive, phasing and cavity tuning systems. 
phase changes across the klystron as the beam voltage and drive are varied. A phase-lock loop around the klystron will minimize these changes. A stable phase reference cable will carry phase information from a pickup in each cavity back to a phase bridge near the rf source. At any time, the phase can be touched up to within an accuracy set by the stability of the phase reference cable by varying the input phase to each klystron until the phase of the signal from. each cavity pickup agrees with a reference phase setting. The reference phase settings are the phases measured for each pickup immediately after a complete phase alignment has been made using the symchrotron-frequency technique.

\section{Control of Longitudinal Instabilities}

The six circulating bunches in PEP can be considered as six coupled harmonic oscillators with six normal modes. The in-phase barycentric (zero mede) phase oscillations of the bunches can be controlled by a feedback loop coupling a signal picked up from the beam back to a varactor diode phase modulator in the input drive to one or more of the klystron groups. In order to damp the other five possible modes, a high-frequency cavity in the proper location is required. When the high-frequency cavity is operating, the time derivative of the voltage seen by each of the bunches is different and the synchrotron oscillation frequency is therefore different for each busish. If this "splitting" of the synchrotron frequencies is sufficiently large, the bunches are effectively decoupled against longitudinal phase oscillations, as has been demonstrated in other storage rings.

Three potential longitudinal instabilities which can arise out of the beambeam interaction have been suggested and studied. ${ }^{22}$ The first occurs only when the colliding beal is cross at an angle, which is not the case in PEP. The second is due to $\approx$ non-zero value of the $\eta$-function at the interaction region $\eta *$. It places 
an upper limit on the value of $\eta^{*}$ which is higher than the value at which PEP will operate. The third occurs when the bunch length is large compared to the value of the $\beta$-function at the interaction region. This is not the case in PEP.

\section{Excitation of Other Cavity Modes}

Because the PEP accelerating structure consists of five coupled resonant cells, there are four modes in addition to the $\pi$ mode in the fundamental pass band of the structure which can interact with the beam. In the PEP ring, the harmonics of the revolution frequency are spaced only $140 \mathrm{kHz}$ apart. Thus there is the possibility that a neighboring harmonic may fall close to one of these other four modes. Tuners will be located in each cell of the structure. By proper adjustment of these tuners, the interaction of the beam with these additional mories can be minimized.

A potentially more serious problem in the design of the PEP rf system is the additional power loss due to the excitation of higher-order cavity modes. Both theoretical and experimental approaches are planned in attacking this problem. It is expected that by proper structure design, the enhanced power loss due to the excitation of higher-order carity modes can be held to a tolerable level.

\section{Vacuum}

\section{Introduction}

The vacuum system proposed for PEP will be similar in design, construction and operation to the system currently in use at SPEAR. ${ }^{23}$ The modifications required for PEP are primarily due to the ilcreased synchrotron power radiated per unit length, which results in increased radiation-induced gas desorption. All design parameters and system features are primarily dictated 
by the pressure requirements to achieve adequate beam lifetimes and low background rates in the experimental areas.

The SPEAR vacuum system has proven satisfactory both in construction and operation. The extruded aluminum chambers, the explosion-bonded aluminumto-stainless-steel transitions and the cleaning and welding techniques developed have produced a reliable system. There have been no detectable vacuum leaks in any of the extruded chambers or transition materiai as received from the fabricators. The system base pressure has reached lorels lower than $2 \times 10^{-9}$ Torr with no in-place bakeout. The distributed ion fumps have operated successfully, demonstrating a pump speed of over $300 \ell /$ sec per meter and have kept the system pressure rise below $6.5 \times 10^{-11}$ Torr per milliampere of stored current.

\section{Vacuum Chamber Construction}

The vacuum chamber in the bending magnets will be an 11-meter -long, 6061T4 aluminum extrusion with an internal cross section as shown in Fig. 17. The inner cross sectional area is deslgned to accommodate the beam-stay-clear region required by beam dynamics. The eynchrotron-radiation absorbing wall will be approximately $10 \mathrm{~mm}$ thick. The absorbing surface will be ridged as in the SPEAR chambers in order to minimize the synchroron-radiation-induced gas desorption. As shown in Fig. 9, a cell vacuum chamber will consist of two bending magnet chambers and two quadrupole chambers. Thu quadrupole chambers will accommodate the required expansion bellows, pusition monitors vacuum gauges, etc.

The segments of the vacuum chamber are joined togetizer with flanges. All flanges larger than $102 \mathrm{~mm}$ in diameter will be standard stainless steel vacuum flanges with copper gaskets. In the main, these will comprise the rolding-pump flanges and the $250-\mathrm{mm}$ flanges at the exds of the chambers. These flanges will 


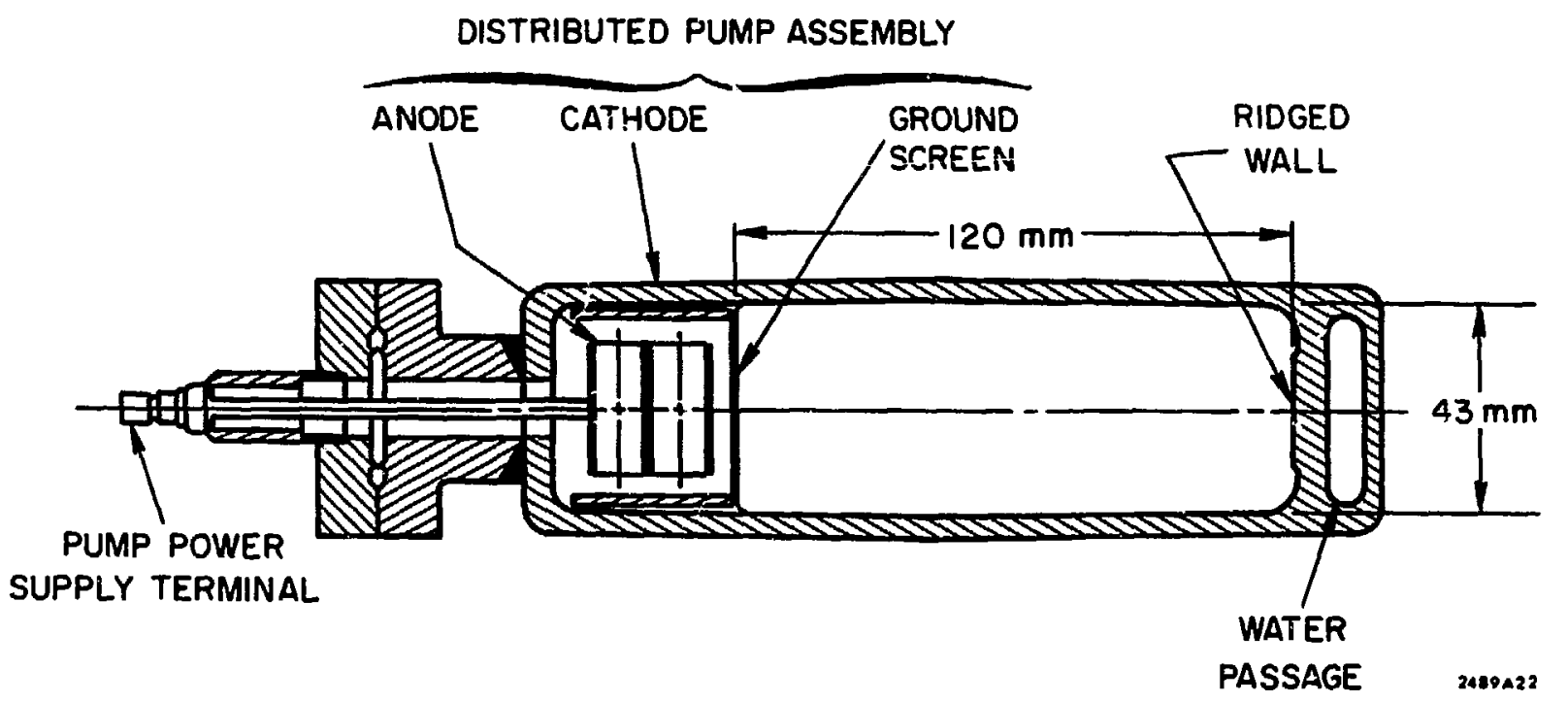

FIG. 17 Cross section of the vacuum chamber for the bending magnets. The distributed ion pump which operates in the fringing field region of the magnet is shown with one of the high-voltage terminals. 
be attached to the aluminum chambers by means of explosively-bonded transition sections like those used in SPEAR. Smaller flanges will be made of solid aluminum.

\section{Synchrotron Radiation}

The surfaces which are subjected to synchrotron radiation require cooling. The heat which must be dissipated by such surfaces is quite intense and represents one of the major factors determining the vacuum system design. Conservative design criteria are necegsary to minimize the danger of a water leak into the vacuum. In addition, intense radlation fluxes create high gas loads due to syachtotron-radiation-induced gas desorption.

At $15 \mathrm{GeV}$ and $100 \mathrm{~mA} \mathrm{~d} . \mathrm{c}$. current in each beam, the total power radiated per heam would be 2.6 MW. The power distribution for the geometry of the vacuum chamber in a main bending cell is plotted in Fig. 18. The maximum linear heat fux for two counter-rotating beams occurs in the region of the chamber which spans the gap between adjacent bending magnets. With two $100 \mathrm{~mA}$ beams stored, this maximurs is about $46 \mathrm{~W} / \mathrm{cm}$. The synchrotron radiation consists of photons with a spectral energy distribution up to well above $80 \mathrm{keV}$ as shown in Fis. 19.

The main gas load during operation is due to synchrotron-radiation-induced desorption which is dominated by a two-step process in which a photoelectron is ejected from the vacuum chamber wall by a synchrotron-radiation photon and subsequently desorbs a gas molecule upon reentering the wall. This gas load is concentrated in the arcs where the synchrotron radiation is produced. Data from SPEAR indicate that it is conservative to extrapolate the desorption rate linearly with beam energy. The total photon desorbed gas lond for the entire ring is estimated to be $10^{-5}$ Torr $-1 / \mathrm{mA}-8 e c$ at $15 \mathrm{GeV}$. 


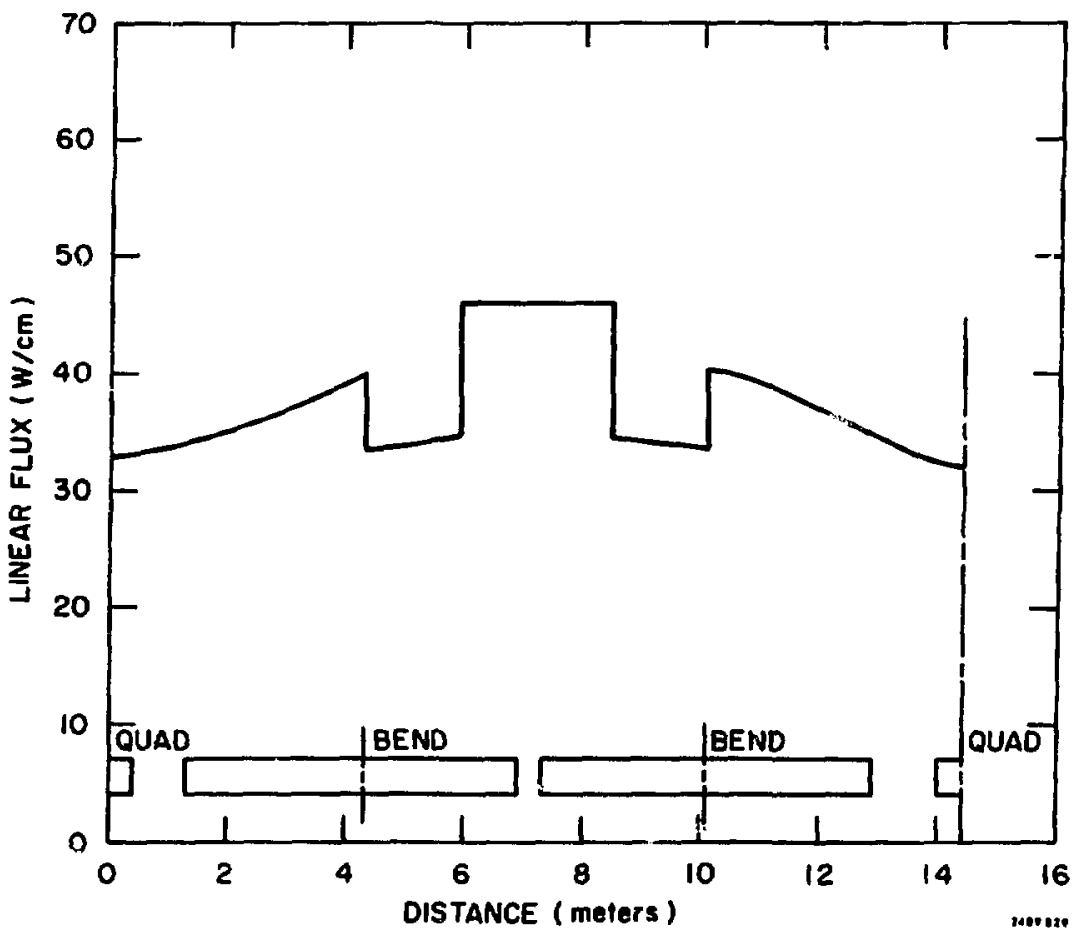

FIG. 18 Power flux due to synchrotron radiation plotted for one-half of a standard cell. The discontinuities are caused by discontinultles in the bencing fleld and by changes in the curvature of the vacuum chamber. 
공

NUMBER of PHOTONS $(\mathrm{sec}-\mathrm{eV})^{-1}$ per milliampere

害

产

흠

ō

흠

믈붕

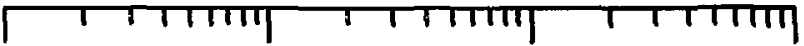

SPECTRAL POWER (W/eV) per milliampere
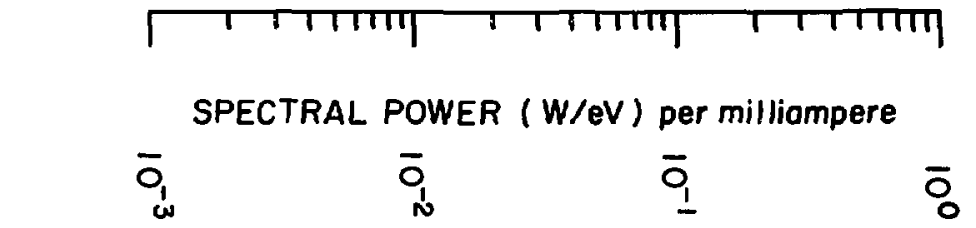

3

(1)

옹

त

虫冒

g is

ż

要

角

물

옹ํํ

$\overline{8}$

3

3

틀

용

ర్

。용

宓臬

용

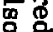

mo

要

옴

뭄

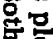

웅

임

要品

D

.

웅

도음

용으

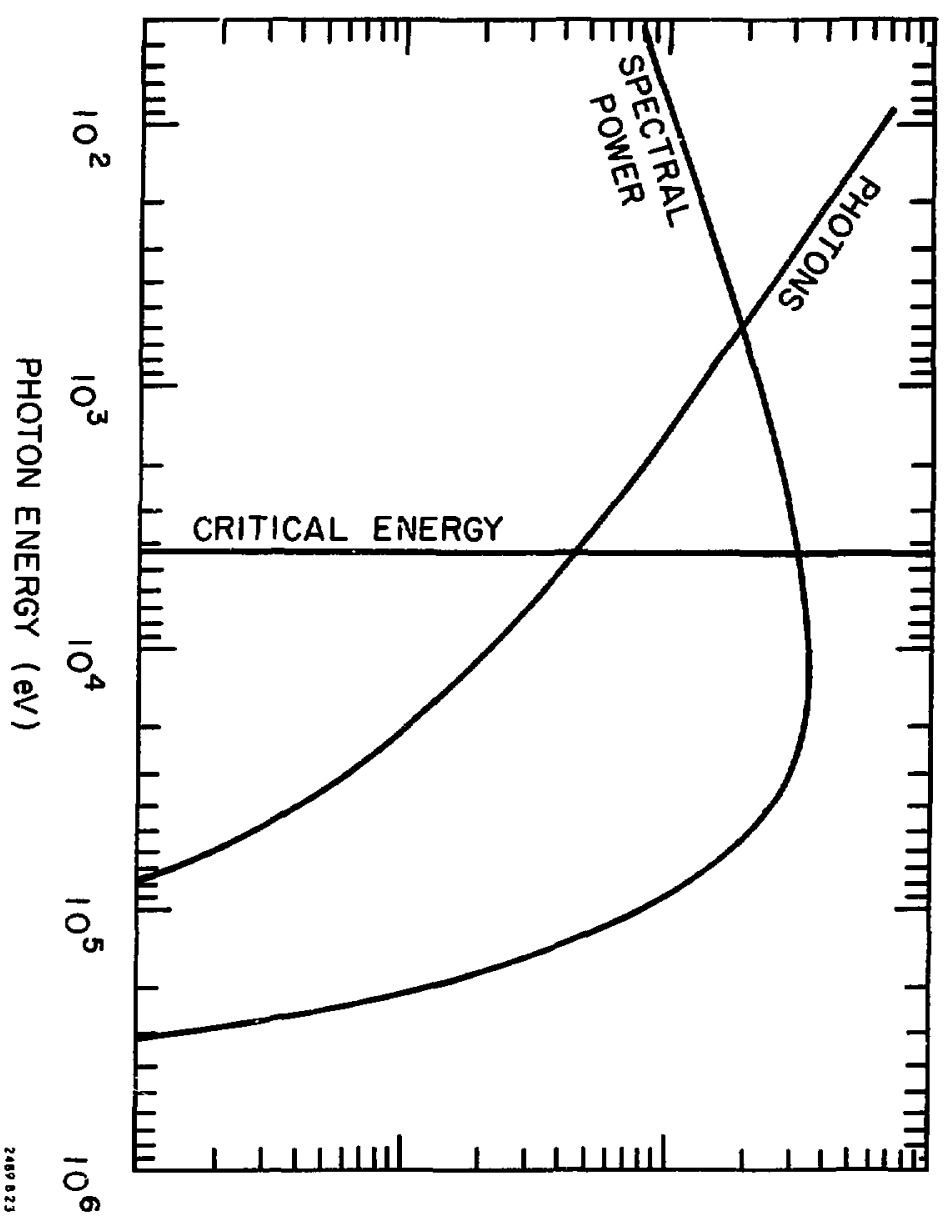




\section{Vacuum Pumps}

The vacuum system is conductance-limited in the bending magnet chambers where the bulk of the outgassing occurs. Distributed sputter-ion pumps of the type which were developed and used for SPEAR will therefore be installed inside the vacuum chamber within the bending magnets. These pumps are rated at $300 \mathrm{\ell} / \mathrm{sec}$ per meter of pump length. Commercially available $100-\ell / \mathrm{sec}$ ion pumps will be mounted at each quadrupole and will maintain the system at a base pressure of $1 \times 10^{-9}$ Torr without a stored beam.

The calculated pressure distribution with two 15-GeV, 100-mA beams circulating is shown in Fig. 20. Based on the above estimate for the total gas load, the maximum pressure will be $5.0 \times 10^{-8}$ Torr and the average pressure will be $2.5 \times 10^{-8}$ Torr.

\section{Instrumentation, Valves, Controls, etc.}

The vacuum system design will incorporate isolation valves, vacuum gauges and pump ports in order to facilitate pumpdown, isolate sections as protection aga inst accidental venting to air and for diagnostics. There will be an in-line valve and a nude pressure gauge in every fourth cell. The valves will be actuated manually, locally or from the control room, or automatically upon indication of pressure rise from the vacuum gauges. Ports with right-angle valves vill be installed in each quadrupole straight section. These valves will be used for pumpdown connections, installation of mechanical or thermocouple gauges, diagnostics and venting. Pumpdown will be accomplished by a combination of coldtrapped pumps and cryo-pumps.

\section{Cooling Water}

Based upon the distribution of synchrotron radiation shown in Fig. 18, the maximum heat flux is 46 watts $/ \mathrm{cm}$. At this flux, the temperature differential 


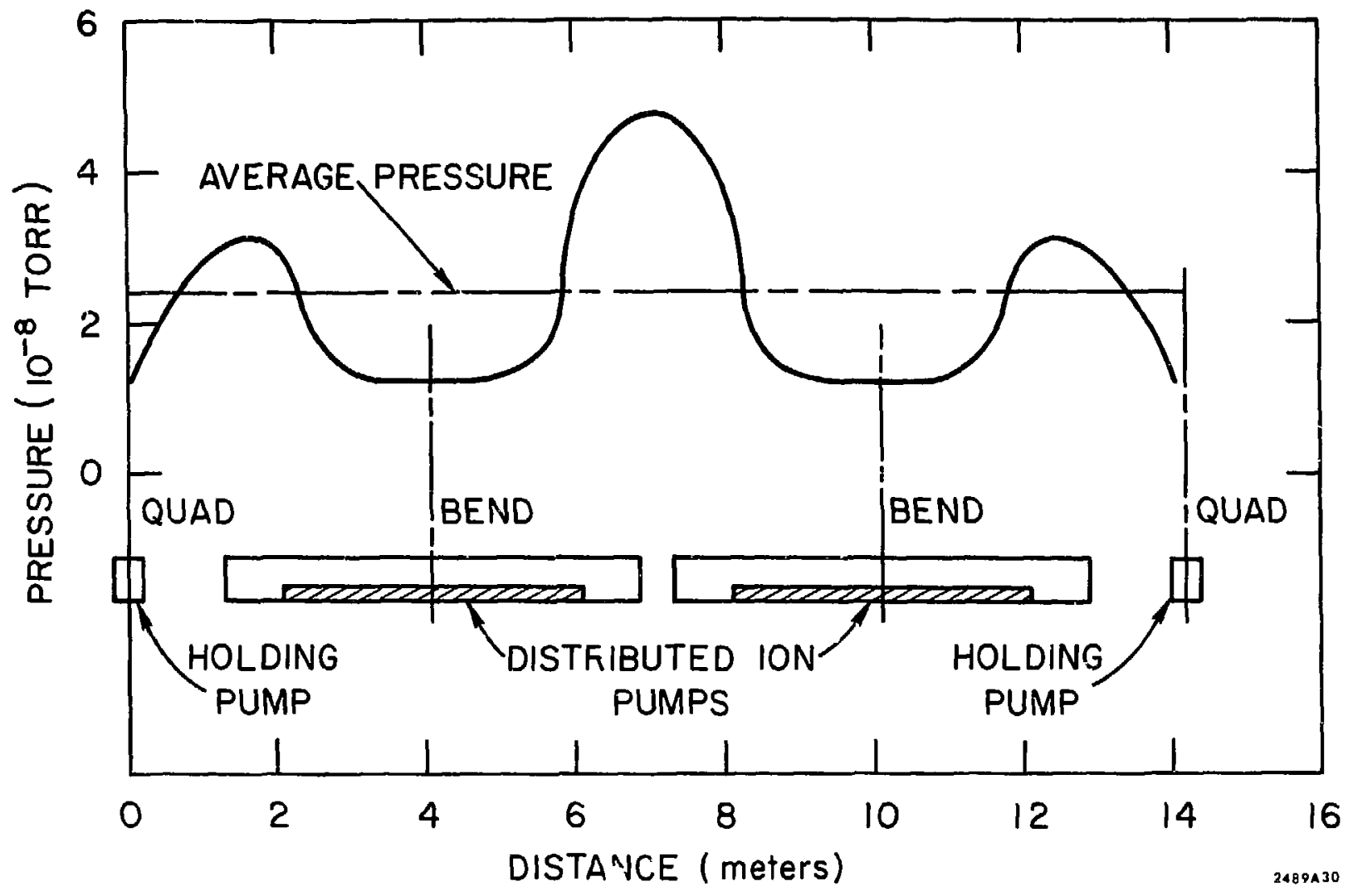

FIG. 20 Pressure distribution in a standard cell with two $100-\mathrm{mA}$ stored beams. Pumping is provided by $100 \mathrm{l} / \mathrm{sec}$ hclding pumps and $1200 \mathrm{\ell} / \mathrm{sec}$ distributed ion pumps located as shown. 
across the water-cooled wall of the chamber is $45^{\circ} \mathrm{C}$ and the temperature differential across the surface film in the water is $26^{\circ} \mathrm{C}$. The water flow rate will be $1.3 \mathrm{l} / \mathrm{sec}$, resulting in a bulk temperature rise of $10^{\circ} \mathrm{C}$. Wate: will be suppliec at $30^{\circ} \mathrm{C}$.

The thickness of the water-cooled web of the vacuum chamber and the area of the cooling water passage is determined by the thermal conditions given above. In order to overcome the pressure drop through the twelve meters of cooling water passage and yet not exceed the allowable stress in the cooled web, the supply water pressure will be 14 ATM.

Because of the intense heat fluxes and the associated heat transfer problems, the vacuum chamber cooling water system must be exceptionally clean and will therefore be separate from the other cooling water systems.

\section{Insertions}

The insertions will be fabricated from 300 -series stainless steel tubing. Provision will be made for adequate water-cooling of areas subjected to synchrotron radiation. The initial design of the insertions, where the main gas load is due to thermal outgassing, will provide for an average pressure of $5 \times 10^{-9} \mathrm{Torr}$. The chambors will be provided with ports to facilitate the addition of pumps adequate to maintain a pressure of $5 \times 10^{-10}$ Torr if required by experimental ucnditions.

\section{Bakeout}

Space will be provided for possible bakeout of the vacuum chambers in place in the sextants if it proves necessary. The bending magnet vertical gap will be adequate to accommodate heater tapes and insulation on the vacu: Im chambers. All chambers will be baked to a temperature of $200^{\circ} \mathrm{C}$ prior to installation in the ring. 
In the insertions, including interaction regions, space will be provided for installatio: t heaters for in-place bakeout capability. The design will provide for repeated thermal cyclings to a temperature of $200^{\circ} \mathrm{C}$. Isolation valves within the insertion are planned as are gauges placed in strategic locations.

\section{PEP Injection}

\section{Injection Rates}

In PEP, as in many recent single-ring $\mathrm{e}^{+} \mathrm{e}^{-}$colliding-beam facilities, very few of the rf buckets are filled. The six PEP interaction regions are illuminated in fact by only three bunches in each beam. The relatively high $\mathrm{rf}$ frequency of $\sim 350 \mathrm{MHz}$ is about the same as that being used for the SPEAR II rf system and implies that the $\mathrm{rf}$ phase acceptance will be of the order of nanoseconds. A special new electron gun has been installed on the two-mile sccelerator to provide very high peak currents in the short $(\sim 1.4 \mathrm{nsec})$ pulses necessary for injection into SPEAR II, and it is equally well suited to PEP. Figure 21 is a plot showing the PEP filling time vs injection energy. The line shows the predicted injection times based on a conservative 50\% injection efficiency and the expected y ields of electrons and positrons with the new gun. Since typical PEP operation could require refilling as often as once per hour, it follows that an acceptable filling time must be a small fraction of an hour. From Fig. 21, it can be seen that this can be achieved at energies above approximately $10 \mathrm{GeV}$ up to the maximum positron energy from the two-mile accelerator, which coincidentally is $15 \mathrm{GeV}$, the design energy of the ring. The maximum injection repetition rate, which is used at the highest injection energies, is $360 \mathrm{pps}$, the full SLAC rata. After filling PEP, the accelerator may return to servicing the regular SLAC experimental program. Switch-over times of the computer-controlled accelerator systems should be negligible. 


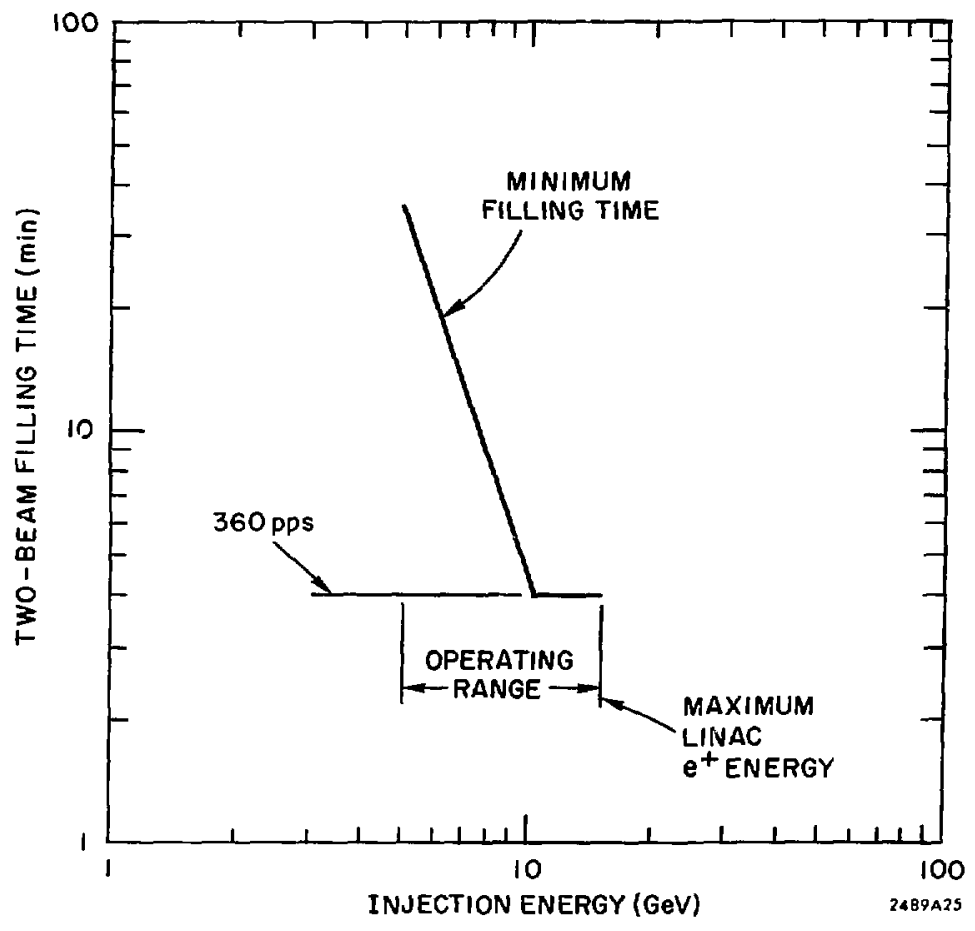

FIG. 21 Filling time vs injection energy. Filling time is the time required to fill both beams to a current of $100 \times[E(\mathrm{GeV}) / 15] \mathrm{mA}$ from a beam of $20 \mathrm{~mA} \mathrm{e}^{+}$and $40 \mathrm{~mA} \mathrm{e}^{-}$in a $1.4 \mathrm{nsec}$ pulse with $50 \%$ capture efficiency. 
At lower filling energies, the damping rates of horizontal betatron oscillations and the energy oscillations (which fall as $1 / \mathbf{E}^{3}$ ) do not permit full utilization of SLAC's repetition rate. However, the design of the injection system is such as to permit the filling of an adjacent bunch while a previously injected bunch is damping. The curve in Fig. 21 reflects this mode of operation.

For storage ring operation at energies below reasonable injection energies, especially below $10 \mathrm{GeV}$, the beam will be stored at the injection energy and then the ring will be ramped down to the operating energy.

\section{Injection Beam Transport System}

The injection lines consist of two beam transport systems, each bending a total of $60^{\circ}$ in the horizontal plane while descending from the accelerator elevation of 77 meters above mean sea level to the ring elevation at approximately 65 meters above mean sea level. The injection lines cross over the storage ring and end up on the inside of the ring at the near ends of the first straight insertions : orth and south of the linac centerline (see Fig. 22). This arrangement, permitting injection from the inside of the ring, avoids severe design complications associated with synchrotron radiation from the counterrotating beam striking the beam entrance window.

Modifications to the accelerator and beam analysis area at the end of Sector 30 will be required to extract alternate bunches of positrons and electrons into the two transport lines. The accelerator sections will be removed from Girder 30-8 and replaced with a 0.5-degree pulsed magnet. When the magnet is pulsed, the beam is injected into the PEP ring; when it is not pulsed, the beam goes through the BSY to the research areas. This magnet requires only one pulser since a single polarity will bend the oppositely charged particles in opposite directions. Following the pulsed magnet, there will be a double septum 


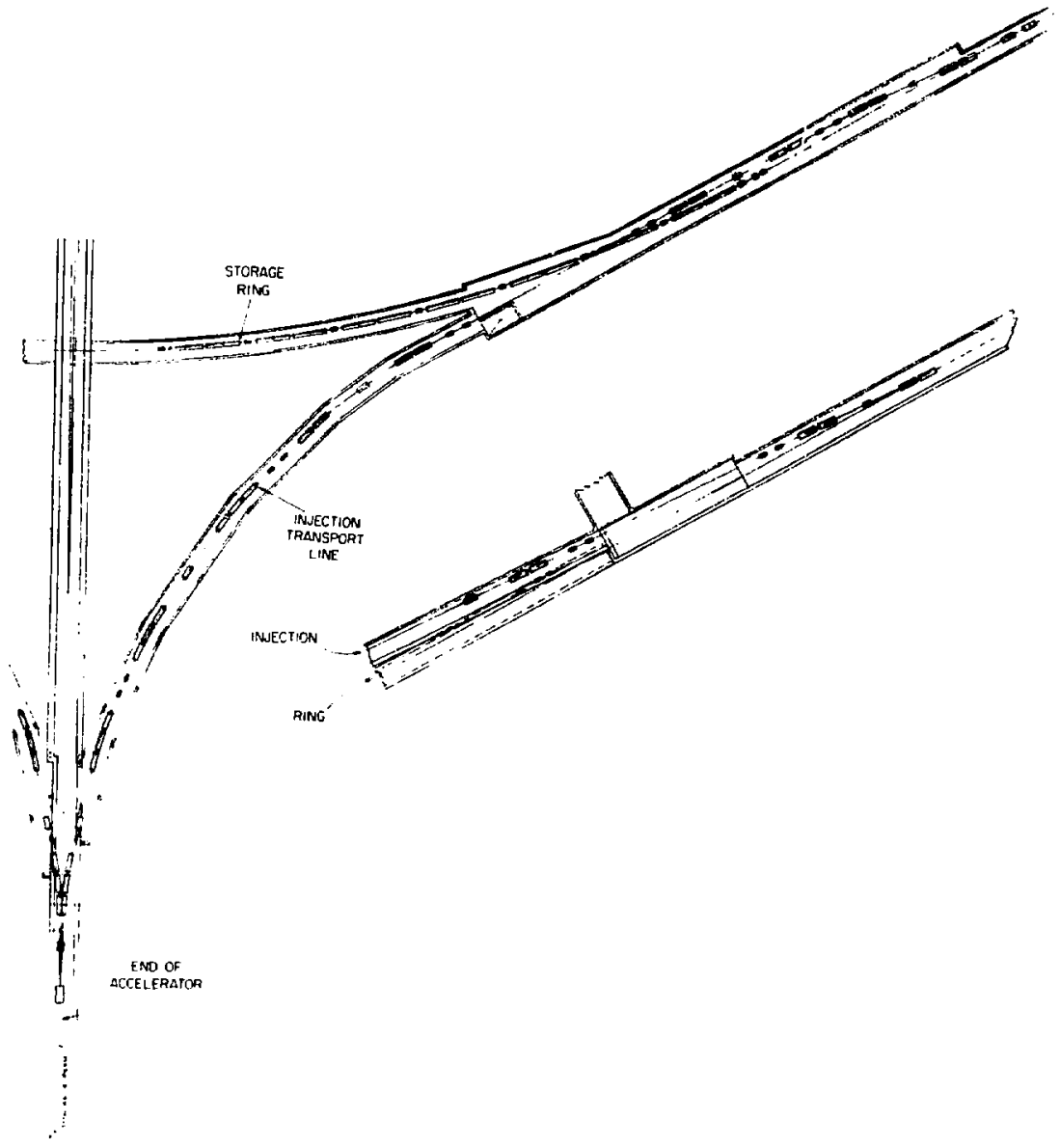

FIG. 22 Injection system beam transport lines. 
magnet, or magnetic slit, which will further bend the two beams away from the accelerator. As the beam on the north side of the accelerator approaches the housing wall, it will be necessary to fit in as strong a bend as can be made so that the angle of penetration through the housing wall is maximized to minimize cost. On the south side, there is adequate room for a total of $12^{\circ}$ bend before the beam enters the housing wall.

Outside the housing wall, the transport systems will continue in tunnels that will be extensions from the main tunnel for the PEP ring. Starting with the first bend magnet after the magnetic slit, all magnets will lie in tilied planes rolled by about $6.5^{\circ}$ around the accelerator axis. Optically, the transport lines may be thought of as achromatic segments joined by matching quadrupole doublets. An energy-defining slit will be installed at the symmetry point of one of the segments. Figure 22 shows the elements of each system based on a threesegment scheme. The bending magnets of the injection transport lines may be significantly stronger than the PEP ring magnets because synchrotron radiation lossos are not important during the single pass that the beam makes through the injection line. The power consumption of these magnets may be high enough to make it appropriate to switch off those parts of the beam lines that follow the first symmetry lens between times of injection to save energy. The beam from the accelerator can be monitored at a low rate at the symmetry lens until injection is called for by PEP control.

At the lower, or PEP, end of each line, another set of bending magnets will be needed to align the beam with the pulsed inflection system. This will require a 5.7-degree vertical achromatic bending system to level the beam into the plane of the ring followed by a horizontal system capable of focusing the injection beam through the inflection system. Since the optimum adjustment of 
the injection beam line may depend somewhat on the operating configuration, these last two systems should be kept independent.

\section{Pulsed Inflection System}

The principle of operation of the inflection system is as follows: A set of air-core pulsed kicker magnets cause a local "beam-bump" in the PEP ring to move the equilibrium orbit closer to a septum magnet. The injected beam, which gets its last deflection from the bending side of the septum, then foins the stored beam with a large horizontal betatron oscillation amplitude. The horizontal betatran tune is chosen so that it will not hit the septum again for four turns. The kicker magnets are turned off well before that time, so that the beam is moved away from the septam, the new particles are stored and damp into the main ensemble of the beam.

This process is continued as many times as necessary until both beams $\left(\mathrm{e}^{+}\right.$and $\mathrm{e}^{-}$) are at the desired current. The kickers are pulsed with a pulse approximately $2 \mu \mathrm{sec}$ long so as to avoid exciting the particles in the other two bunches, the bunches being spaced apart by $\sim 2.4 \mu s e c$. In this way, it is possible to consider the three bunches as three separate beams which can be injected simultaneously without watting for each to damp separately.

The pulsed kicker magnets need to bump the beam only by the amount required $b y$ the injected particles to clear the septum after making a complete horizcntal betatron phase excursion. The betatron phase acceptance of the septum is shown in Fig. 23. Assuming a 1-mm-wide septum and a 1-mm-wide beam, a kick of 0.7-1.0 cus should be adequate. Nominal kicks less than 1 milliradian are adequate for this purpose in the 28-meter space that has been allocated to inflection. 


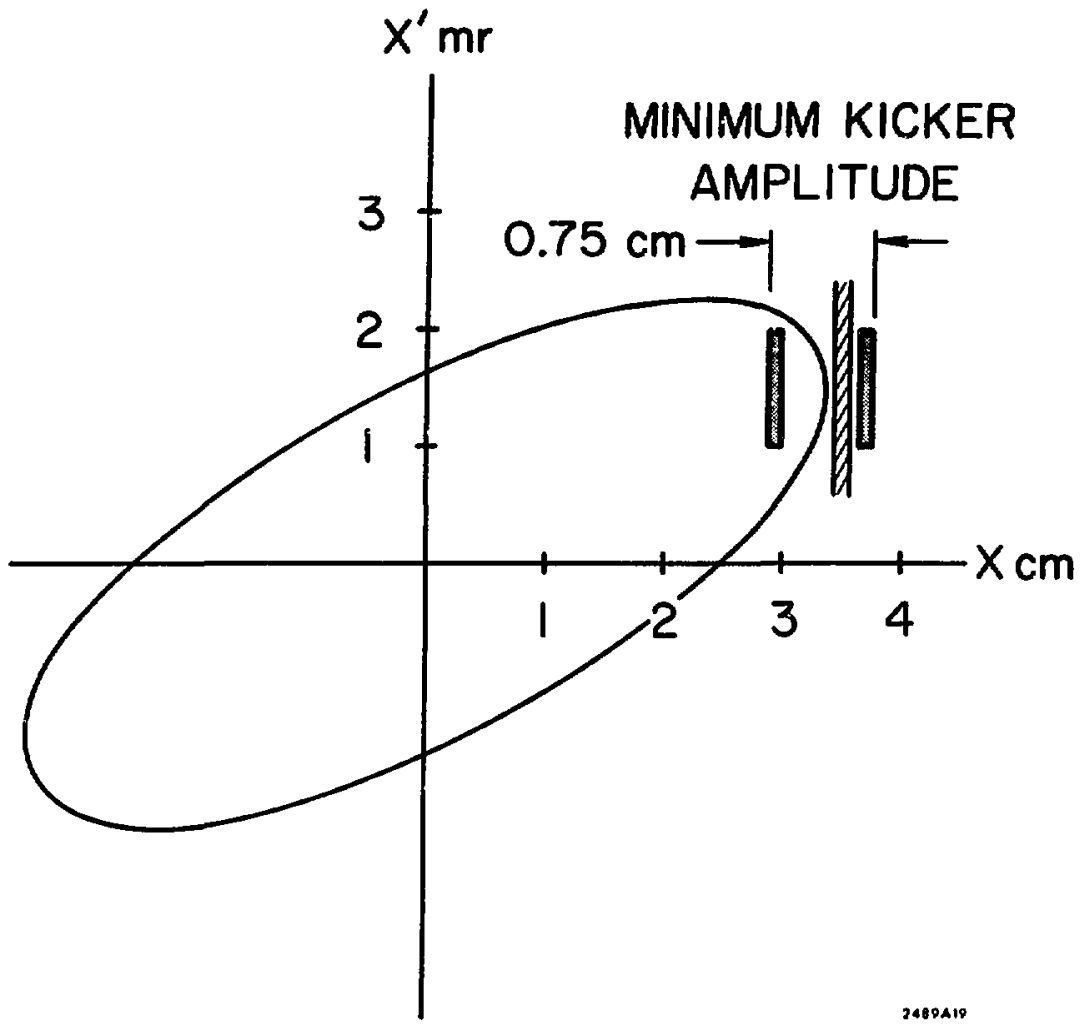

FIG. 23 Horizontal betatron phase plot showing septum location. 
It is desirable to compress the inflection system into a minimum length of the straight section. This is helpful for PEP Stage I and becomes orucial for PEP Stage II when extra bonding magnets are required which severely restrict the available space. A special space-saving feature of the proposed design is the combination of the middle pulsed kicker magnet with the last septum in a single physical element. A formed-current-aheet maguet for thls purpose is shown in Fig. 24. The smaller tube with a somewhat higher field forms the septum magnet for the incoming beam. The magnet design computer program GFUN was used to arrive at the tentative configuration shown in Fig. 24. Further design work with the computer could be done to optimize the configuration for dc. However, because tise fields in the device depend on eddy-currents, the next appropriate step is to make measurements on a low-power test model.

Immediately upstream in the injection transport line from the combination septum-kicker is an Iron-core septum magnet. Thls magnet is required to make a bend of approximately 12 milliradians. It will also provide the most convenient point for final horizontal steering of the injected beam since the combination kicker-septum is locked to the field required to match the beam-bump of the other pair of kicker magnets. The septum magnet will be pulsed to parmit higher currents in the septum which must be thin to clear the beam. On the other hand, a pulsed septum magnet may have difficulty in reaching the design field at $360 \mathrm{pps}$, so some development work is indicated for the $\mathrm{S} 2$ design.

A third, larger, septum magnet will operate in a dc mode. Together with a reversed horizontal magnet just upstream of this system matches the horizontal $\eta$ function of the injected beam to the $\eta$ function of the ring at injection. 


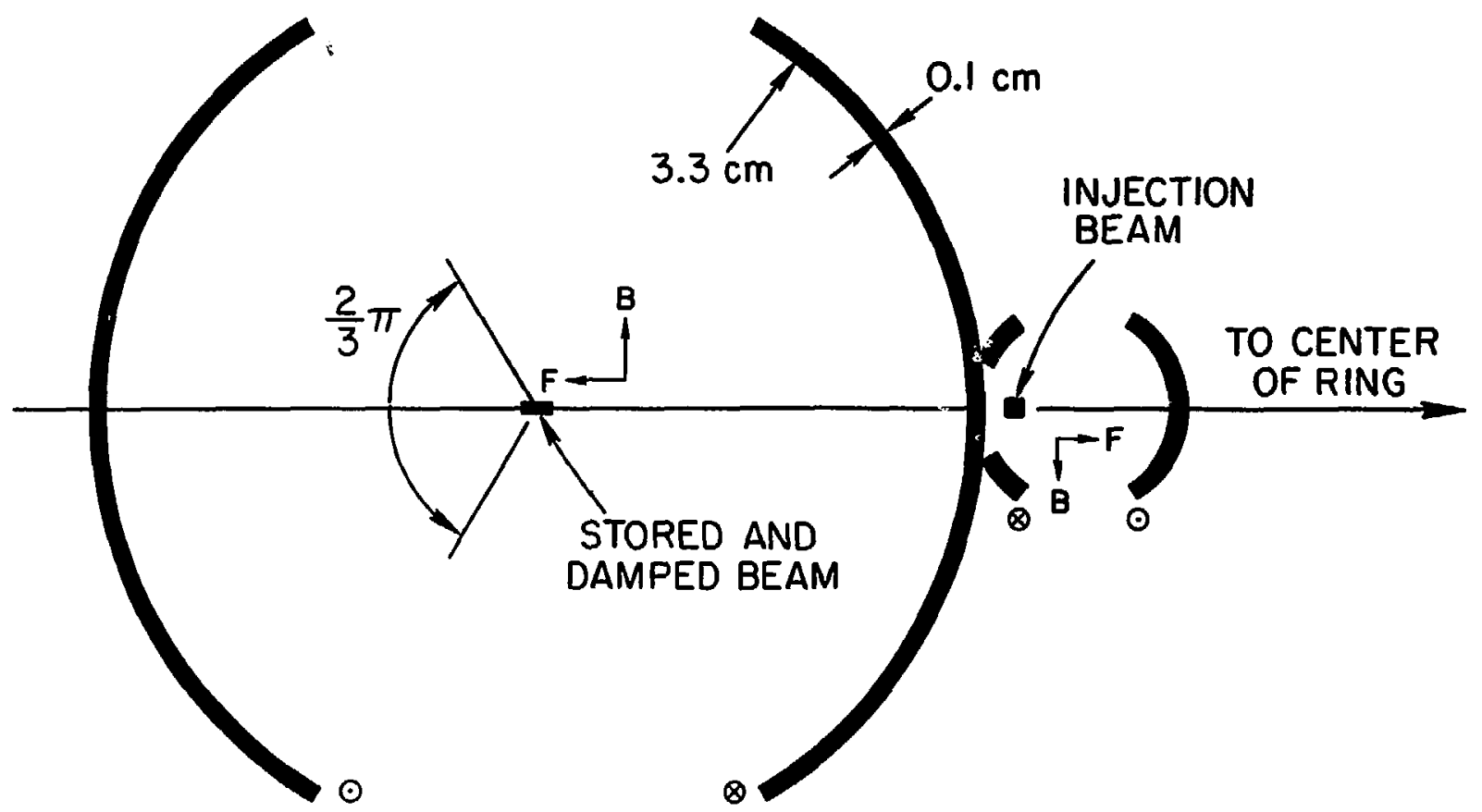

$2489 A 18$

FIG. 24 Schematic of combined kicker-septum magnet. The injected beam is kicked in the opposite direction from the stored beam. All conductors are assumed to be the same thickness and operate with the same current density. They are about $2 \mathrm{~m}$ long and use a pulse of approximately 5000 amperes for $15-\mathrm{GeV}$ injection. 


\section{E. Instrumentation Monitoring and Control}

\section{General}

The storage ring system, like the accelerator, is too spread out for a single data-collection point to be practical. Data will, therefore, be collected at a number of places, edited and compressed as much as possible and then transmitted to the control room. These collection centers will be located at the ends of the six arcs and will where possible be appendages to equipment buildings for the rf system, magnet supplies, etc., from which many of the signals will arise. The control room, which is near one of the injection points, Is well located to permit a short path for direct transmission of fast monitoring signals from the injection lines.

To reduce the amount of cabl:-ig, signals generated within the bending sextants will be wired only to instrumentation racks in the nearest data center. Interlock signals will be summarized and then wired directly to the equipment they must control. The remaining signals will be edited by a small computer and sent by serial link to the control room. The computer in the data-collection area will have provision for graphic displays, thus eliminating the need for extensive alarm and annunciator panels.

\section{Control System}

The control system will be strongly dependent upon computers. The central element will be a "large minicomputer" which will provide task synchronization between the peripheral elements, and manage intercommunication. It need not have elaborate computational abllity. It will have three types of "peripherals". The first type is the "data-collection" computer, at least one per interaction area, which provides all connections to the real world. The second is a console 
manager, one for each operating position, which provides all operator interfaces. The third peripheral will be a moderately sized computational computer which will carry out large-scale calculations and provide file management and programming aids. A link to the SLAC IBM triplex computer system may supplement this computer.

\section{Timing}

Much of the instrumentation will require timing signals at the circulation frequency of the ring. The timing system will divide the $358-\mathrm{MHz}$ operating frequency to $140 \mathrm{kHz}$ and distribute a reference signal to each data area. Timing repeaters will provide separate channels with individual delays for each instrument. Timing signals are required for gating the beam monitors and feedback systems, for injection, for displays in the control room and for the experimenters.

\section{Magnet and Guidance Controls}

The controllers for magnet power supplies will be standardized as much as possible. They will include a digital-to-analog vonverter attached to each power supply regulatcr. All of the converters will be of the same aesign and power supply regulators will be designed to accept signals in the same range; e.g., 0-10 V. Each power supply will have an individual controller. The ganging of controls for magnets that have identical currents, but are too far apart to be in series, will be accomplished in the software of the computer system.

Remotely-controlled high-voltage supplies for the electrostatic guidance plates will be provided. Their controllers will be similar to the magnet power supply controllers. 


\section{Rf System Control}

The rf system will be largely self-contained; for example, it will contain internal logic for phasing on command. The control system will provide for operator's instructions for phasing and for control of rf-voltage amplitude, and will monitor the general health of the rf system components.

\section{Interlocks and Miscellaneous}

The personn trols to control access to the arcs and the interaction areas and will ensure that no beam may be injected or circulated if any area can be occupied.

Analog and status signals for vacuum gauges and pumps will be read into the computer system. Fast valves will be controlled by direct inter-connection from the vacuum gauges.

Thermocouples to monitor the temperatures in the arc and interaction region vacuurn chambers will be monitored by the computer system. Thermocouple and vacuum-gauge signals will be displayed during bakeout on a graphic display terminal at the nearest data-collection area.

\section{Beam Monitoring}

The synchrotron light monitor will consist of the necessary optics and sensors required to generate a TV image of the beam and provide beam profile information and the bunch-lengthing measurement. The light emitted from the two counter-rotating beams will be collected separately. Photodiodes will be used to measure the average current in each beam independently. These sensors will be calibrated from a single toroidal dc current transformer which will be capable of measuring the total current $\left(e^{+}+e^{-}\right)$in the ring to an accuracy of $0.1 \mathrm{~mA}$. Calibration will be done with a single beam in the ring. 
The beam-position-monitoring system is similar in design to the SPEAR system. ${ }^{24}$ It will measure the position of the beam in the $r$ ing at approximately 80 positions. Each monitor will consist of four buttons in the vacuum system which are capacitively coupled to the beam. The amplitudes of the signals produced by the buttons are compared to generate the beam position information.

Two luminosity monitors, one a small-angle, high-rate system and the other a large-angle $\left(10^{\circ}-20^{\circ}\right)$, low-rate system, will be provided.

Fast strip-line monitors for the stored beam and injection beam monitors will also be provided. 


\section{PHYSICAL PLANT}

\section{A. Beam Housing and Site Improvement}

1. Tunnel Design and Construction

The ring will consist of six 60 -degree arcs, each $231 \mathrm{~m}$ long, and six $130 \mathrm{~m}$ straight sections where interactions will occur. (See Fig. 1.) It will be located symmetrically about the axis of the accelerator with the western-most point approximately $100 \mathrm{~m}$ downstream from the end of the accelerator. The terrain slopes downward from the accelerator axis in both north and south directious so that the interaction regions, which are off the axis, will generally lie in areas of lower elevation. Some segments of the ring will be in areas low enough to permit cut-and-cover construction. Those parts deeper underground will require bored tunnels.

The ring which is horizontal, is at an elevation of approximately $65 \mathrm{~m}$ above mean sea level. It crosses under the beam switchyard about $11 \mathrm{~m}$ below the accelerator beam. As shown in Fig. 1, the ring will circumscribe the present research yard and extend slightly beyond the present south boundary of the $\mathrm{AEC}^{\prime} \mathrm{s}$ lease. An additional ten to thirteen acres of undeveloped property will have to be leased from Stanford University.

The beam transport tunnels, through which electrons and positrons are brought into the ring, will start at the end of the accelerator and branch away and downward, crossing over the ring to insertion points from the inside of the ring, as shown in Fig. 1. An alcove will be added to the housing in a suitable location for a future synchrotron radiation research facility.

For convenient reference, the twelve parts of the hexagonal ring are termed Regions 1 through 12, in analogy to the face of a clock. Region 1 is the northeast arc, Region 2 is the northeast straight section, Region 3 the eastern arc 
and so on, as shown in Fig. 25. Region 1, parts of Regions 2 and 4, all of Regions 5, 6 and 7 and most of Regions 8 and 12 are situated in areas of relatively low ground and can be constructed economically by cut-and-cover methods. The remainder, about half of the total circumference, will be constructed by boring tunnels. The total length of the tunnel will be $2166 \mathrm{~m}$, of which about $1100 \mathrm{~m}$ will be tunneled, about $800 \mathrm{~m}$ will be constructed by cut-and-cover techniques and about $260 \mathrm{~m}$ will be used for the experimental halls in Interaction Regions 2, 4, 6, 8 and 12. The Region 10 interaction area will be contained in the normal tunnel section where accelerator physics measurements will be performed.

The two beam transport lines from the end of the accelerator enter the ring housing through enlarged tunnel sections at each end of Region 9. The 170-m balance of the two housings for the beam transport runs will be constructed of bored tunnels which will be somewhat smaller than the normal ring tunnel section.

The storage ring will be positioned high in the tunnel and suspended from the concrete lining. The tunnel is designed for eventual inclusion of a proton ring. (See Appendix A.) The proton storage ring would occupy a middle height and the electron-positron storage ring would be remounted to alternate above and below it, crossing it in a vertical plane at the interaction points. The tunnel will be capable of supporting the electron-positron ring from the ceiling and supporting both rings on the floor. In the bored tunnel areas a circular housing will be constructed, $3.3 \mathrm{~m}$ in diameter, as shown in Fig. 26. A rectangular section $3.3 \mathrm{~m}$ wide by $2.7 \mathrm{~m}$ high is planned in the cut-and-cover areas. The access aisle will be on the outside of the ring. 


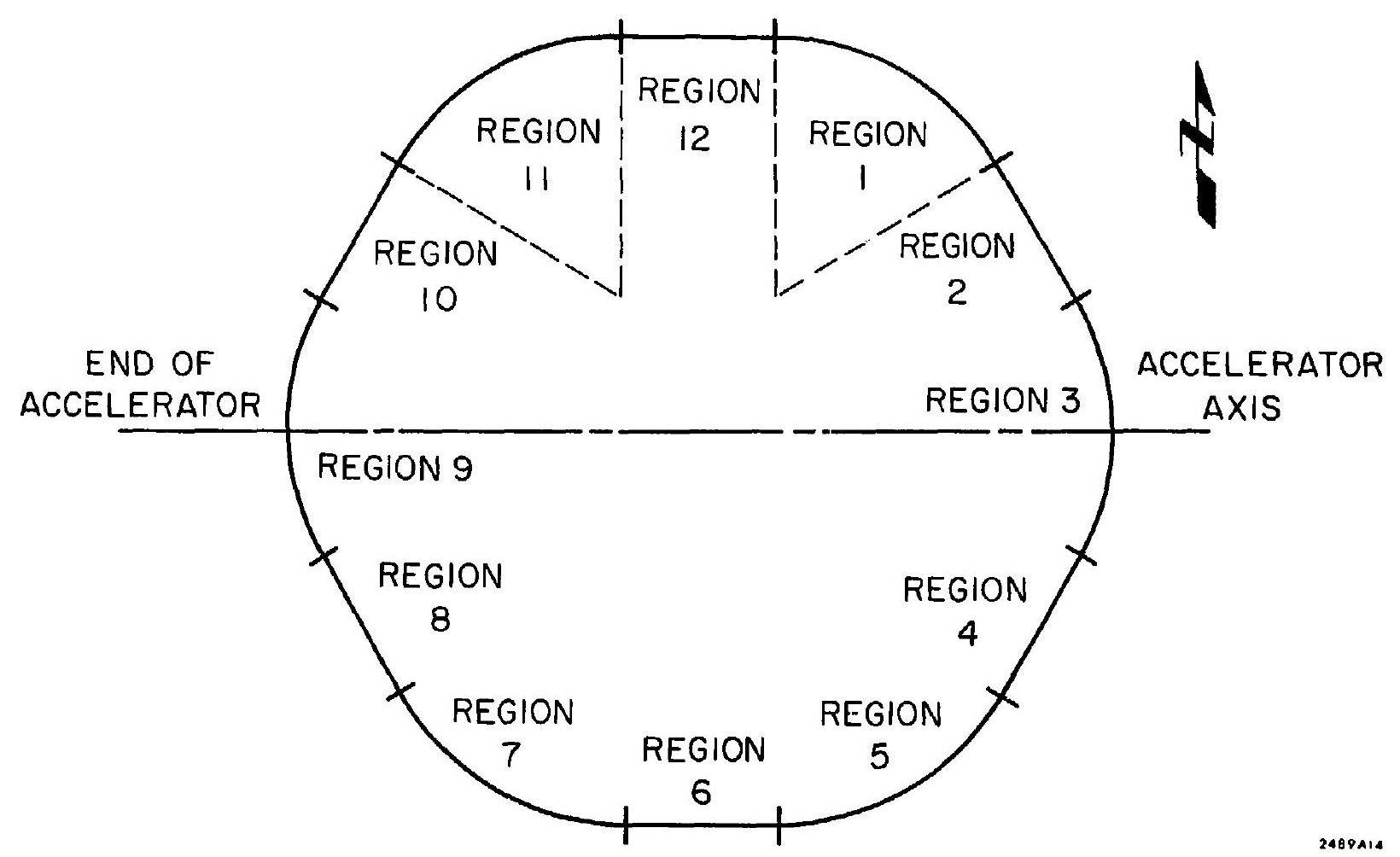

FIG. 25 PEP region numbering scheme. The accelerator beam is deïned to run from west to east with Region 12 on the north side. 


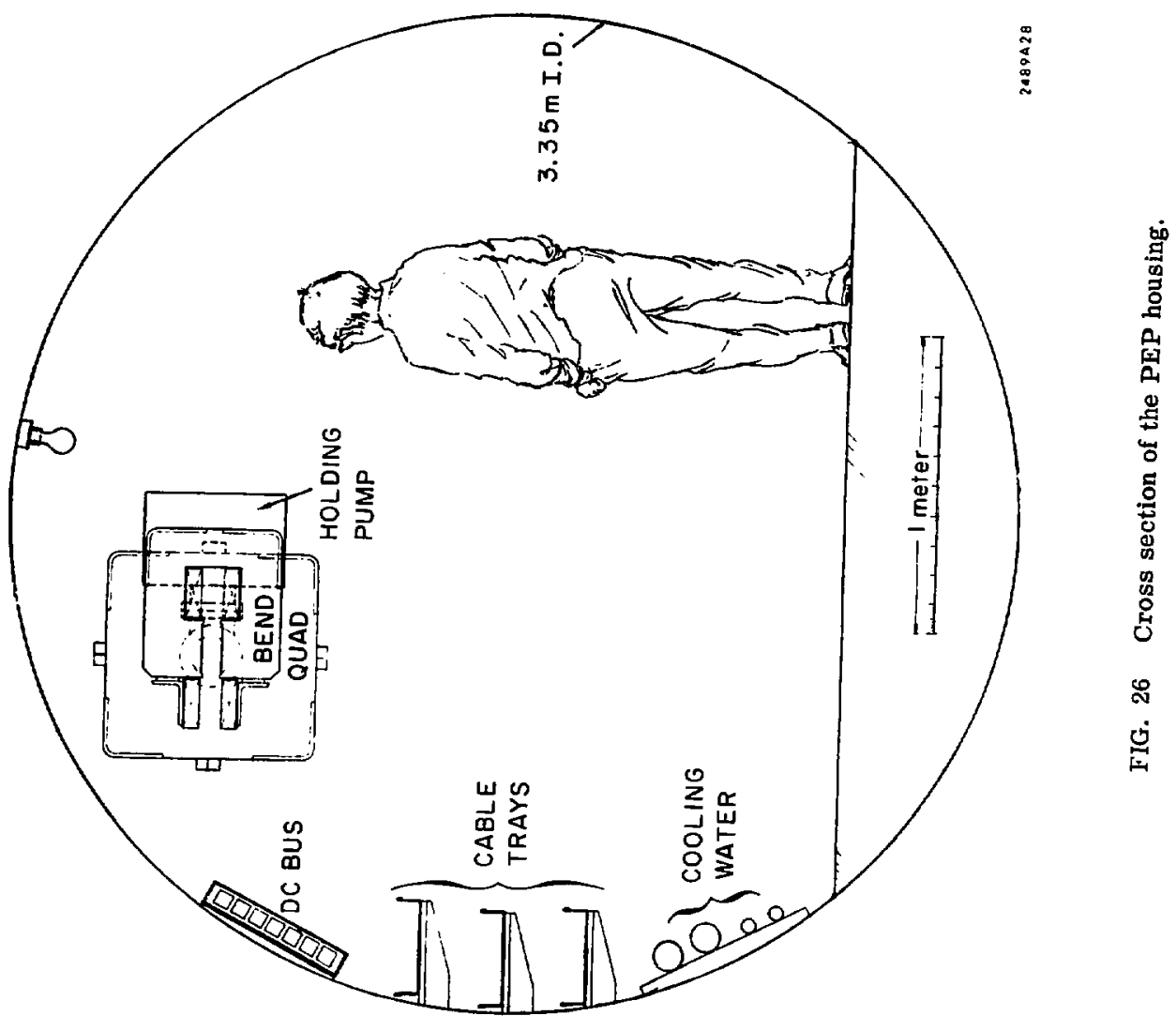


The ring will be level, requiring water intrusions or spills to be drained by pumping. The drainage system will be contained in the floor. The tuanel lining will be painted white to reduce dust generation and to provide good lighting levels. Forced ventilation will provide adequate air movement during personnel occupancy periods. Electrical services, including lighting, receptacle and 480-V welding power, will be mounted on the walls at the inside of the ring. Two supply-and-return low-conductivity-water systems, ons for magnet cooling and one for vacuum chamber cooling, will also be wall-mounted. I \& C cabling will be run in trays.

The contractor for the bored tunnels will be allowed some latitude in shape and size of the finished tunnels to permit the use of readily available equipment. The site geology is predominately miocene sandstone, but some clays, shales and gravels exist. (See Appendix B.) The material is in general far better than the muds and clays to which the term "soft ground" usually refers. Safety requirements will probably demand the use of a shield and steel sets. Lagging will be used both for safety and to provide reaction against the shield's tunneling thrust. The beam transport tunnels of the two injection systems are situated on grades and intersect both the accelerator housing and the storage ring housing at flat angles. About $200 \mathrm{~m}$ of tunnel will be enlarged for the injection transport systems. Traditional mining methods will be used to construct the beam transport tunnels.

At the elevation of the ring, some ground water is to be expected; however, the site is in an area of low porosities and water flow is not expected to be great.

Prior to final design, extensive test boring will be done along the ring alignment to familiarize bidders with conditions to expect in tunneling. The 
design and construction techniques will include means of minimizing noise, dirt and visual impact on the nearby residential communities.

\section{Roads}

The site topography offers little difficulty in the construction of roads. No heavy earthwork will be required other than that necessary to provide benches for roads climbing the sides of the shielding fill. The existing road connecting SLAC to Alpine Road is typical of the roads planned. It is 24 feet wide with 5-foot shoulders and has held up well under heavy use during SLAC's initial construction. This connecting trafficway will be relocated to cross over the ring shielding fill in Region 5. All interaction areas except Region 10 will have access roads. For the most part the grades will be limited to $6 \%$. The project will require an addition of about 2000 linear meters of roads to SLAC's existing system.

\section{Earthwork}

Edges of earthwork sections will be generously rounded to blend into the naturally rolling landscape. Erosion control treatment which will provide quick grass growth will be used. Temporary silting ponds will be provided in drainage ways to prevent silt flow into the City of Menlo Park's drainage systems to the north and into the horse-stable area to the south.

\section{Drainage}

Interaction facilities will be situated in open excavated basins in the terrain with the experimental equipment pits too low for gravity drainage. Highly reliable pumping systems will be installed. Paved interceptor channels will be constructed to divert concentratior.s of storm water to natural channels to avoid development of erosion gullies. 


\section{Sanitary Sewers}

Sewage from the northern part of the ring will flow by gravity to SLAC's existing metering station on the Menlo Park Sanitary District system. Sewage from the southern part will be piped to an existing sewer built some years ago by the University, also on the Sanitary District's system.

\section{Landscaping}

The part of the ring site that is presently undeveloped consists of grassy rolling hills studded with occasional oak trees. PEP construction will require removal of only about five trees. Professional landscape design will be called upon to enhance the area with small groves of trees, but no landscaping needing extensive maintenance is contemplated.

\section{B. Shielding Reguirements}

\section{Introduction}

The first stage of the construction of PEP will consist of an electronpositron storage ring. The underground housings will be designed to be compatible with the future addition of a proton storage ring. Because the production of neutrons by proton interactions is some three orders of magnitude greater than that due to electron interactions, and, in addition, because the energy of stored protons will be about $200 \mathrm{GeV}$ as compared to $15 \mathrm{GeV}$ for the stored electrons and positrons, the total shielding requirements will be determined by beam losses from the future proton storage ring.

The shielding design and operation of the proton storage ring will be predicated upon the maintenance of external radiation levels below:

(a) 5 millirem per year at any point on the SLAC boundary;

(b) 5 millirem per year at any point outside the facility shielding;

(c) less than 1 millirem at any point on the SLAC boundary for any single maximum credible accident, i.e., losing the entire beam at the worst point. 
The thickness of radiation shielding, the amount of radioactivity induced in accelerator components and surrounding structures and the degree of radiation damage to components and materials depends, to a first approximation, upon the product of energy and intensity of beam losses.

There are several possible sources of beam loss:

(a) Injection, acceleration and transition losses;

(b) Gas scattering;

(c) Beam instabilities;

(d) Beam scrapers;

(e) Controlled dumping;

(f) Accidental beam dumping.

At the present time, it is possible to give only speculative estimates of the magnitudes of these losses for the proton storage ring. Somewhat conservative estimates of beam loss are therefore used in this report. Eventually, experience gained in the operation of ESCAR, the Experimental Superconducting Accelerator Research Project at the Lawrence Berkeley Laboratory, will help define some of these beam losses more accurately.

The quality of the stored proton beam will slowly deteriorate with time and it will be necessary to refill the proton ring at regular intervals. The ring will be refilled when the luminosity falls significantly, perhaps by a factor of two. It should be noted that a reduction in luminosity need not necessarily result from a corresponding loss of protons from the stored beam. When the ring is to be refilled, the remaining stored beam will be decelerated to an energy of $20 \mathrm{GeV}$ and extracted from the ring into a dump. 
The three beam loss mechanisms which dominate are losses on scrapers, controlled dumping and accidental losses. Beam scrapers and dumps will be sited in regions of large overburdens. In other regions, accidental dumps will determine the shielding requirements. Experience at the CERN ISR would indicate that accidental beam losses of the entire stored beam will be rare occurances.

\section{Shielding for Curved Sections}

The overhead shielding requirement is determined by the hadron cascade generated in the shield by escaping protons. The minimum overburden planned for the ring tunnel is $5.5 \mathrm{~m}$ of earth each $\left(1100 \mathrm{gm} \mathrm{cm}^{-2}\right)$. Calculations based upon extrapolations from experience at the CERN PS show that this overburden plus the added shielding provided by the magnets will permit $\mathbf{5 0}$ high-energy dumps at full intensity $\left(3.6 \times 10^{13}\right.$ protons) without exceeding the radiation limit of 1.5 rem per year at the shield surface. The corresponding dose equivalent at the site boundary, assumed 100 meters distance, would be $\sim 1$ millirem.

Muons, produced by the decay of pions and kaons in flight, will be strongly peaked in the forward direction. They are not a problem in the vertical plane. It is possible that they will penetrate the shielding in the horizontal plane at curved sections of the tunnel, however. For electrons and positrons, the maximum muon energy that may be produced is $15 \mathrm{GeV}$. In this case, any muons will always be stopped by the combined shielding effects of magnet steel and earth around PEP. When a proton storage ring is added, the maximum muon energy is $200 \mathrm{GeV}$. In this case, the muons have sufficient energy to penetrate the lateral shielding in the area of Regions 4 through 7 on the south side of the storage ring. Preliminary estimates indicate that less than 5 millirem would be 
produced at the site boundary by ten full-intensity beam dumps at the single most sensitive location.

\section{Shielding for Experimental Areas}

At such time as a proton beam may be introduced into the ring it will be necessary to construct heavier roof $s$ on the halls, adequate to support weight equal to $4 \mathrm{~m}$ of earth. This will result in higher, thicker banks at the walls as well as shielding the roof and side walls. Further personnel protection will be afforded by fencing around roof areas to prevent access when the ring is operating.

The alcoves at the sides of the experimental halls will be covered with earth shielding in depths adequate for eventual proton beam operation. This will cause most of the areas adjacent to the walls of the halls to be well shielded. Since the housings must be designed to support the anticipated eventual full height of shielding, and since the earth excavated from the tunnel has to be disposed of anyway, there is no extra cost in providing this level of shielding during the initial construction of the electron-positron storage ring.

Portable shielding blocks are expected to be made available from the supplies of the two laboratories.

\section{Experimental Areas}

The wide range of experiments possible at PEP in both its initial phase as an $e^{+} e^{-}$storage ring and with the later addition of a proton ring dictates the need for variety and fiexibility in the types of experimental areas developed with the machine. Since the experiments contemplated for this device may remain in place for relatively long periods of time and because they are so intimately connected with the operation of the storage ring, it is necessary to develop and outfit the maximum number of experimental areas that is feasible in order to 
carry out the strongest possible experimental program. The planned site for PEP offers convenient access to five of the six interaction regions. Thus, it is proposed that five experimental areas be developed in a manner suitable for experiments in the first stage of PEP. The sixth one, in Region 10, will have access for only relatively 2 mall experimental setups such as those needed for accelerator physics and luminosity monitoring.

The complement of experimental areas described here is regarded as typical; however, a Summer Study will be held in 1974 on the subject of PEP experimentation, and the details of the experimental areas may change.

The primary constraint on the experimental areas, imposed by the magnet configuration, is the length of the interaction region drift section, which will be 20 meters. This is the distance between the final focusing elements of the storage ring and is the space in which most experimental equipment will be mounted. The beams collide at the center of the interaction area.

It is proposed that two of the experimental areas be of the basic design shown in Fig. 27. These so-called "Standard" areas are seen as general purpose facilities which will accommodate many of the experiments planned for PEP, including those involving a future proton ring. The tuáic design of this area is an $8-\mathrm{m} \times 20-\mathrm{m}$ pit with $4 \mathrm{~m}$ of clearance above and below the beam line. On either side of the pit is a platform $4 \mathrm{~m}$ wide and $3 \mathrm{~m}$ below beam elevation. These dimensions are determined by examining some of the experiments envisaged for standard areas such as tests of quantum electrodynamics, various studies of hadron production and searches for weak interaction effects.

Typical quantum electrodynamics experiments involve the detection of backto-back high-momentum pairs of electrons, photons and muons by means of shower calorimetry, momentum analysis and range-absorption. Shower 

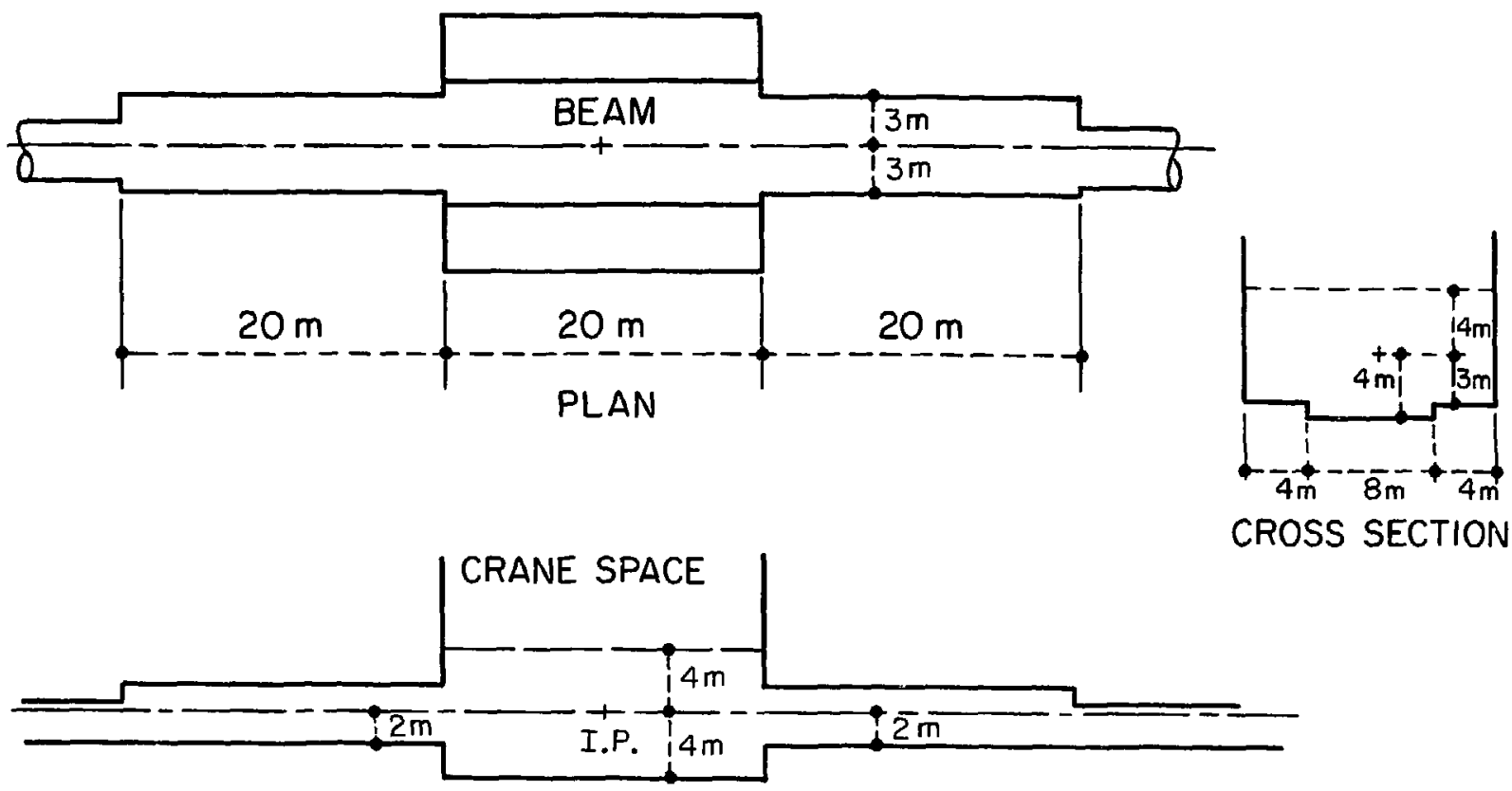

LONGITUDINAL SECTION

2489AI?

FIG. 27 "Standard" interaction area with alcoves. 
calorimeters for $15-\mathrm{GeV}$ electrons and photons will be at least $1 \mathrm{~m}$ deep in the particle direction and additional tracking detectors and support structure will bring the total radial dimension up to $3 \mathrm{~m}$. In precision muon experiments, the basic radial dimension is also $3 \mathrm{~m}$, much of it made up by iron absorber for eliminating all particles except energetic muons. In all cases, symmetry about the beam axis and the capability of measuring at all azimuthal angles (because of beam polarization) are required. The Standard areas are well suited to all of these conditions.

Hadron production experiments will need to detect and measure the direction, momentum and particle type for hadrons over the entire solid angle, and the hadrons will have momenta anywhere from 0 to $15 \mathrm{GeV} / \mathrm{c}$. This large task will be carried out by a variety of experimental setups using different techniques. Certainly, one important experiment will be to measure the total hadronic cross section and to see if the mean momentum per hadron remains bounded at about $500 \mathrm{MeV} / \mathrm{c}$ as seen thus far at SPEAR. A device similar to the SPEAR magnetic detector could be used. The overall radius of this detector, $3 \mathrm{~m}$, is set by the desired momentum resolution $(\delta \mathrm{p} / \mathrm{p} \sim \mathrm{p}(\mathrm{GeV} / \mathrm{c}) / 20, \mathrm{~B}=4 \mathrm{kG})$ and the flight path necessary to separate protons and $\mathrm{K}$-mesons by time-of-flight techniques up to a momentum of $1.2 \mathrm{GeV} / \mathrm{c}$. Spectrometers for better momentum resolution and better high-momentum particle identification demand larger amounts of space in the horizontal plane. The $\pm 8 \mathrm{~m}$ horizontal dimension of the Standard area seems adequate for spectrometers equipped with Cerenkov counters and conventional magnets capable of measuring hadron momenta up to $15 \mathrm{GeV} / \mathrm{c}$ with precision of a few percent. With superconducting magnets better resolution can be attained. Forward angle experiments on hadron prochuction and weak interactions might be performed with a large bending magnet, such as 
a cyclotron magnet placed directly over the interaction region and detectors arranged along the beam line. Again, the Standard area is suitable for a great variety of hadron production experiments.

There are certain physical processes which will be very important to measure in both the $e^{+} e^{-}$and $e-p$ storage rings where particles are emitted at extremely small angles with respect to the beam directions. To detect these particles, it will be necessary to place detectors very near the beam line and well downstream from the interaction point. The characteristic angle of the outgoing electrons in a $2 \gamma$ process, for example, is $\sqrt{\mathrm{m}_{\mathrm{e}} / \mathrm{E}_{\text {beam }}}$ with approximately half of the electrons being outside this angle. Electron and positron counters placed $10-20 \mathrm{~cm}$ from the beam and up to $30 \mathrm{~m}$ from the interaction point will be reasonably efficient for detecting or tagging $2 \gamma$ events at the design beam energy of $15 \mathrm{GeV}$. (See Section II G.) Since tagging of these processes may be desirable in a large number of experiments, the Standard areas will have $6-\mathrm{m}$-wide alcoves which extend to $\pm 30 \mathrm{~m}$ from the interaction point along the storage ring with $2-m$ vertical clearance.

The third area, designated "Standard Plus", will have the same transverse dimensions as a Standard experimental area, but the pit will be extended along the beam direction to a total length of $30 \mathrm{~m}$ and the forward angle alcoves will be omitted. This extension is provided mainly for weak interaction experiments where they may be significant interference between the single photon exchange amplitude and weak amplitude in the forward direction for processes such as muon pair production. (See Section II C.) Rather involved forward-angle experiments providing for ranging out muons (using 10-15 $\mathrm{m}$ of iron) to measure their polarization are accommodated by the Standard Plus area. A schematic drawing of this experimental area is given in Fig. 28 . 


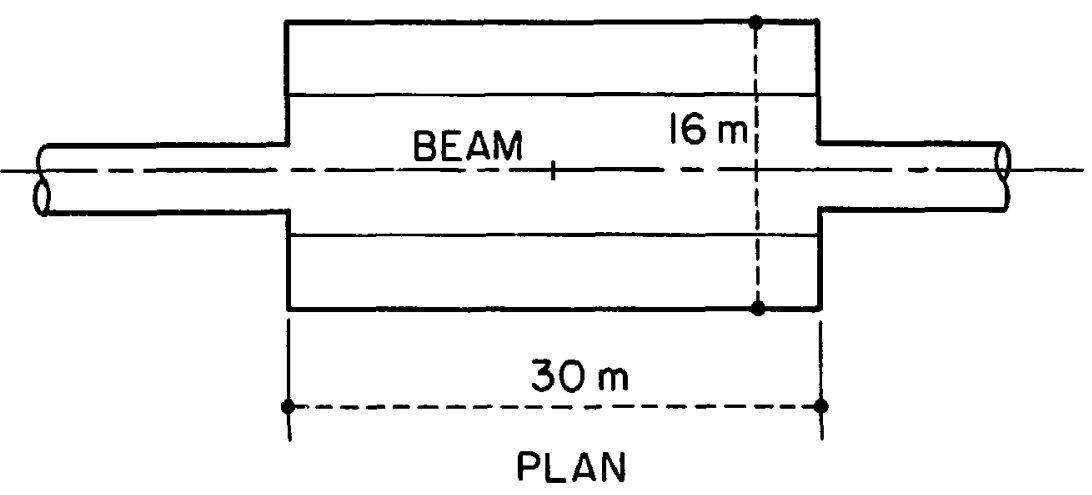

1
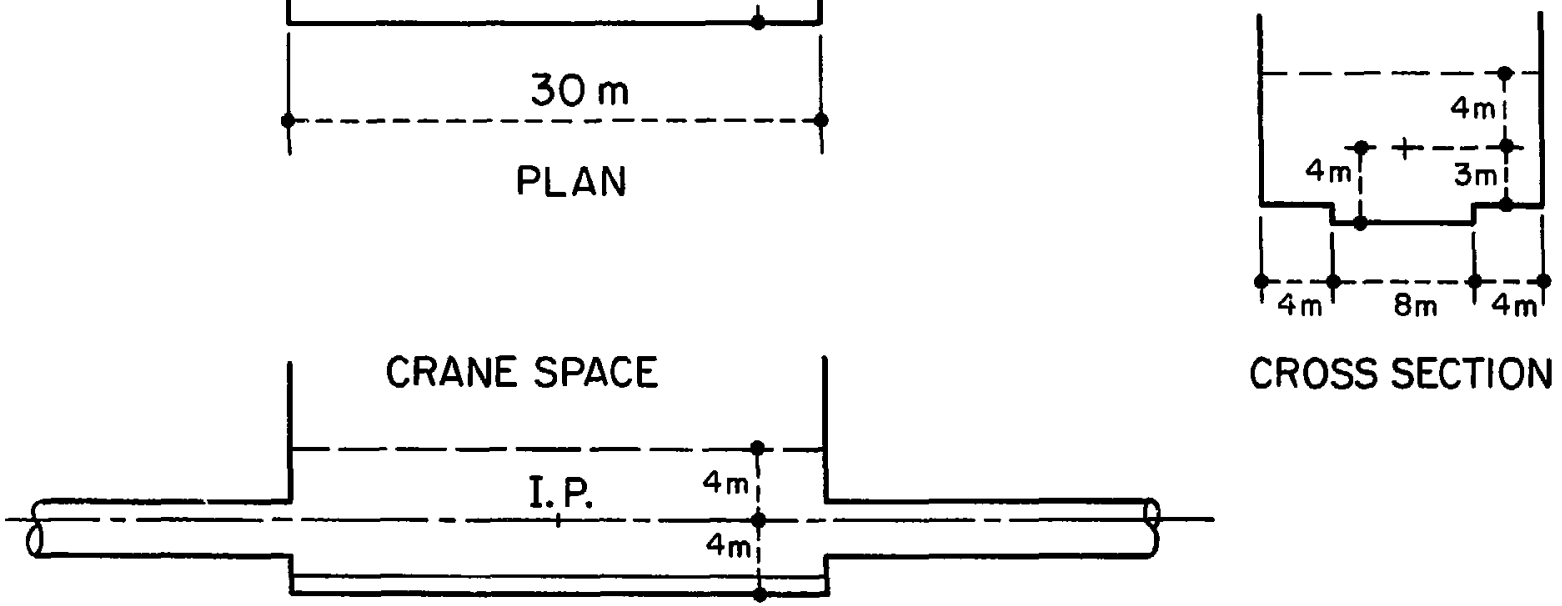
CROSS SECTION

LONGITUDINAL SECTION 
The fourth experimental area, designated "Big $I^{\prime}$, is shown in Fig. 29 and could be dedicated to a large $4 \pi$-steradian magnetic detector or some other large device as yet unconceived. The layout of the experimental pit area at the interaction region is largely determined by the geometry of a large cylindrical magnetic detector similar to the one in current use at SPEAR, except with a superconducting coil and possibly also provisions for calorimetry to give additional information on energetic hadrons. If calorimetry were included, the return-yoke thickness would probably consist of many interaction lengths of iron with interspersed layers of detectors to sample the multiplicity of the hadron shower and to measure the position and width of the shower cone. A total thickness of $2 \mathrm{~m}$ of iron, corresponding to 10 to 15 interaction lengths, seems adequate. Part of the absorber for the calorimeter might well be the superconducting material used for generating the strong magnetic field in the inner volume of the detector.

The inner volume would contain planes (or cylinders) of wire spark chambers in a solenoidal field of perhaps 30 kilogauss. The diameter necessary for this part of the detector depends upon the field strength, the resolution desired to accomplish the event reconstruction, and, of course, the dynamics of the reactions. Of particular importance is the frequency with which very-high-transversemomentum particles are produced. In weak interactions, for example, the detection of such particles may be crucial. In any case, an inner diameter of $4 \mathrm{~m}$ would seem to be a reasonable upper limit, which implies a maximum overall diameter of about $8 \mathrm{~m}$, or $\pm 4 \mathrm{~m}$ from beam line. The pit region as indicated has clearances of $\pm 6 \mathrm{~m}$ vertically, and horizontally $8 \mathrm{~m}$ and $12 \mathrm{~m}$ on elther side of center lice. These dimensions allow some extra space for work around the shell and for contingencies. 


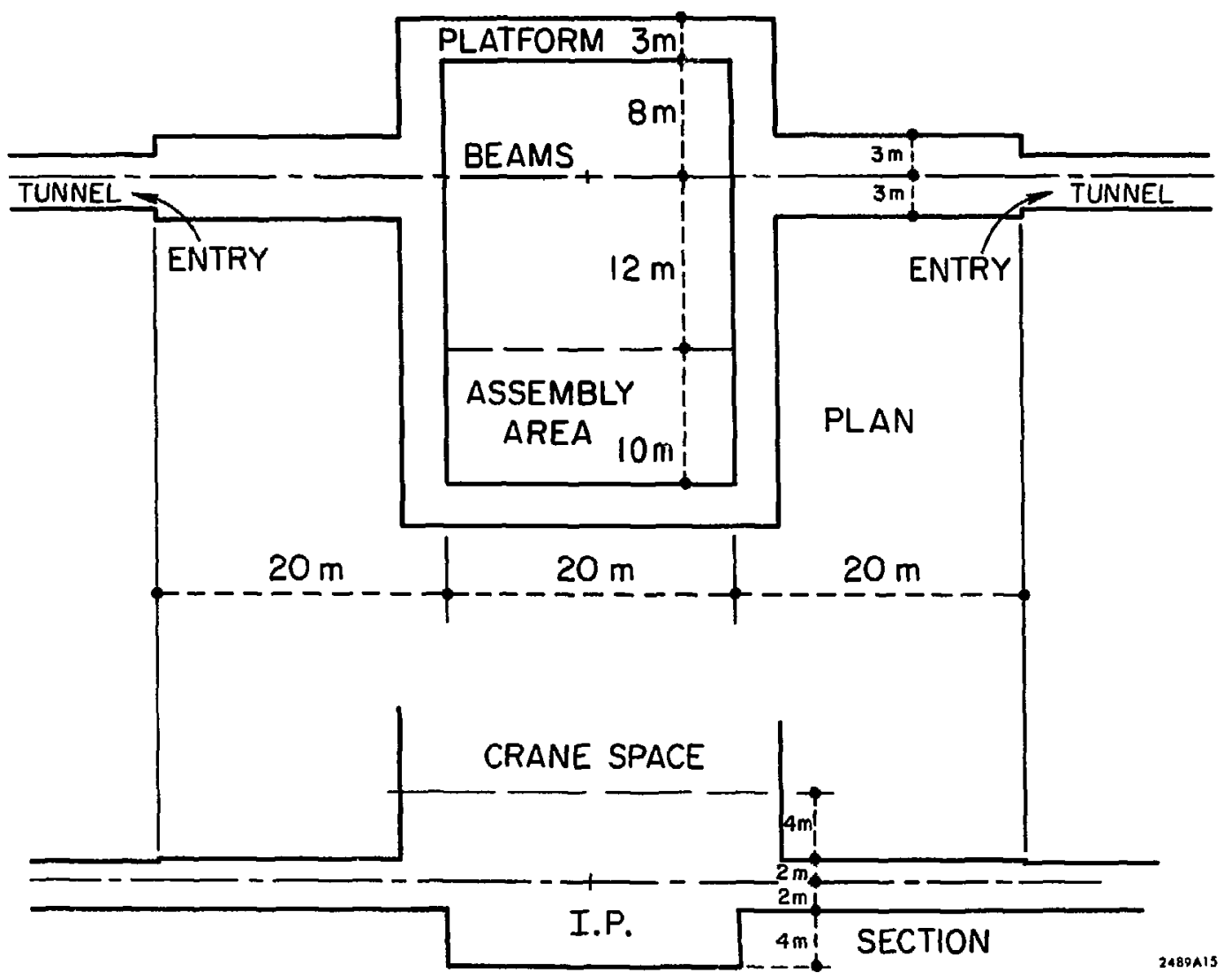

FIG. 29 "Big I" interaction area for a large magnetic detector. 
The fifth experimental area is designed with an eye to future potential expansion. Initially, it will have the same dimensions as the Standard experimental area except that the alcoves will be omitted. In addition, the ends of the pits will be made in such a manner that either one or both can later be easily extended to provide additional experimental space downstream of the proton beam for various possible e-p devices such as another $4 \pi$-steradian magnetic detector, a high-resolution spectrometer to measure inclusive spectra with good particle identification up to large angles, a multiparticle spectrometer along the electron beam for photoproduction studies or a multiparticle spectrometer along the proton direction.

\section{Experimental Structures and Facilities}

Principal functions of the research structures will be to provide sheltered space of adequate dimensions, radiation shielding, means of handling apparatus, and to serve as focal points for utilities and communications. Since the ring itself will be well underground, the interaction facilities will be set deep into the ground. Highly reliable drainage systems will be provided to avoid ground water intmusion and to provide for storm water drainage.

In order to be compatible with the possible future adition of a proton ring, the structures will be capable of modification, in reasonable time and at reasonable expense, to withstand application of increased radiation shielding loads when the proton capability is added. The initial construction will have heavy concrete foundations and concrete walls capable of retaining earth and supporting heavy roof loads at a future date. Roofs may be of relatively light construction for the electron-positron storage ring, although evolution of structural design may indicate desirability of heavler roof construction in order to provide strutting or diaphragm strength to withstand earth pressures against the walls. 
Figure 30 shows typical features of interaction facility structures. The illustration shows structures rectangular in plan, although this is not intended to rule out the use of curved walls to resist earth pressure if such were demonstrated to provide construction economy.

Terrain is the dominant factor in determining the interaction points at which the various types of research facilities will be located. Referring to Fig. 25, Region 10 will be deep below the surface and therefore will be used for accelerator physics. No structure other than the normal beam housing tunnel will be located there. Region 4 is in high ground with a steep slope, requiring the selection of a facility of modest dimensions. "Standard Plus", having no alcoves, seems to be well suited. Regions 8 and 6 are on lower ground, suitable for "Big $\mathrm{I}$ " and the potentially expandable facility, respectively. Regions 2 and 12 are in terrain suitable for the construction of the two Standard experimental halls.

Conventional house power suitable for research operations will be provided, as will fire protection systems. The halls will be heated and ventilated, but no air-conditioning is planned.

Experimental hall interiors will be of unfinished character, similar to present SLAC experimental facilities. However, careful architectural attention will be given to those building exteriors exposed to view, in order to minimize visual impact to neighboring areas. Excess excavated material will be used for landscaped screening fills. 


\section{Utilities}

The maximum power demand of the electron-positron storage ring and experimental apparatus is estimated to be $26 \mathrm{MW}$, divided as follows:

$\begin{array}{lc}\text { Transport and Injection } & 2 \mathrm{MW} \\ \text { Ring Magnets } & 3 \\ \text { Rf } & 11 \\ \text { Experimental Equipment } & 5 \\ \text { House Power } & 3 \\ \text { Cooling Towers } & 2\end{array}$

SLAC's 30-MW standby power system (60 kV) can be activated to meet the PEP demand. Two $12-\mathrm{kV}$ underground feeders will be run from the existing 60-kVto-12-kV transformer.

The installed capacity will be $36 \mathrm{MW}$. While the distribution system can provide $3 \mathrm{MW}$ to each of the five experimental areas, it is expected that the total experimental equipment load will not exceed $5 \mathrm{MW}$ at any time.

Except for experimental areas,low-conductivity water (LCW) will be provided for the installed power capacity. One megawatt of cooling capacity will be installed at each experimental area. Some cooling capacity will be provided by existing SLAC cooling towers. A new cooling tower will be installed to handle the remaining load. Ring components will be cooled by relatively small local LCW eystems. Cooling tower water will be distributed around the ring to cool the closed-loop LCW systems.

Compressed air and dry gaseous nitrogen will be supplied all around the ring and to the experimental areas. The nitrogen is needed for purging and letting-up vacuum components. Natural gas will be supplied for heating the experimental halls. Domestic water will be provided in the experimental halls for personnel use as well as for fire protection. 


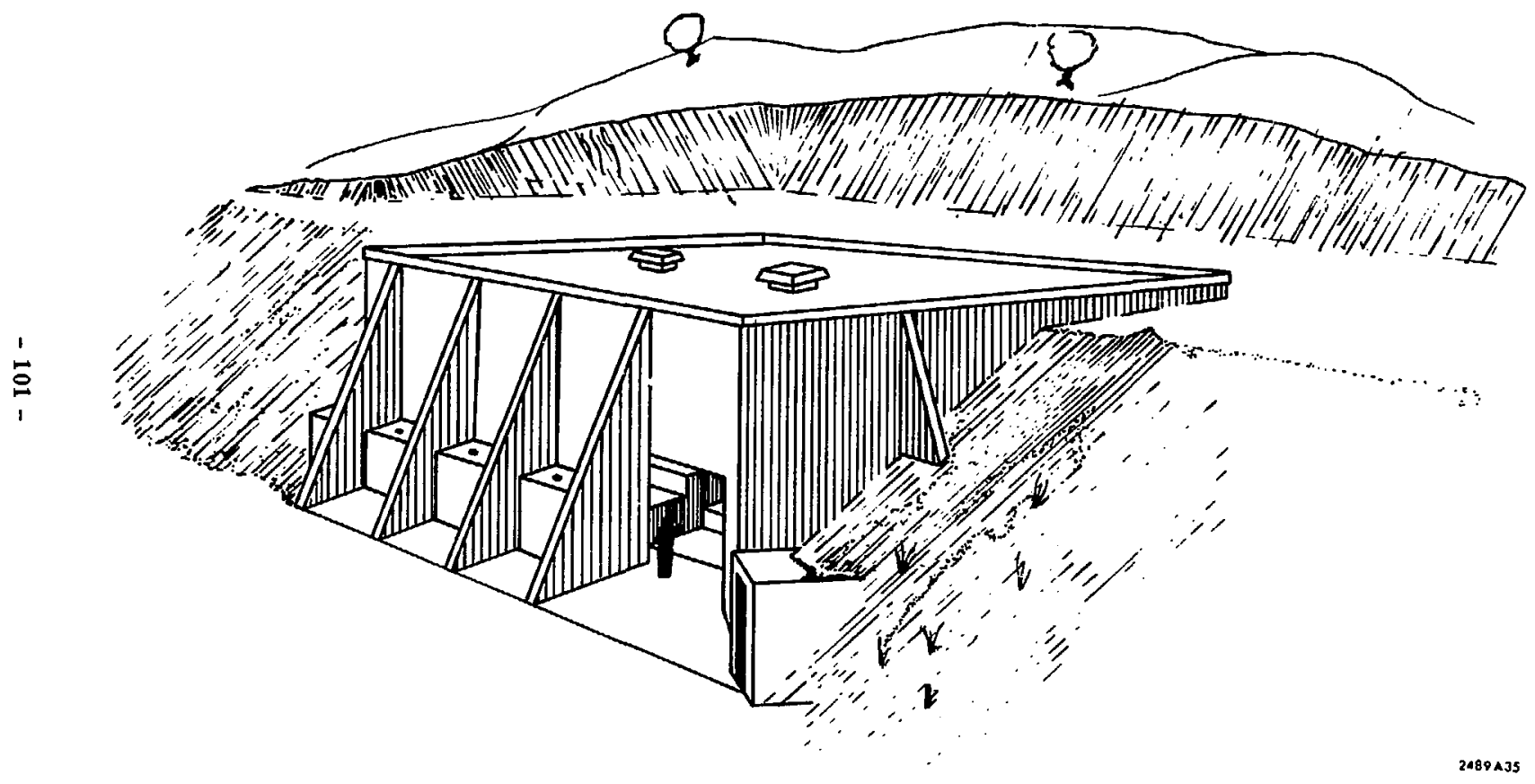

FIG. 30 View of an experimental hall showing shielding configuration proposed for $\mathrm{e}^{+} \mathrm{e}^{-}$rings. 


\section{COST ESTIMATES AND SCHEDULE}

In this section are presented the construction cost estimate and the construction schedule for the PEP electron-positron colliding-beam facility. The schedule is based on the expenditure of $\$ 900,000$ in Fiscal Year 1975 for partial advanced design and assumes full authorization of the project in Fiscal Year 1976. Beam tests are scheduled to begin in April 1979.

The estimated total cost expressed in 1974 dollars is $\$ 53.3$ million. Recent experience dictates that a substantial allowance should be made for price escalation; accordingly we have chosen a rate of seven percent per year applied to the uncosted balance over the life of the project. The total escalation allowance is $\$ 8.5$ million, leading to a grand total estimated cost of $\$ 61.8$ million.

Contingenc ies are applied at $15 \%$ of conventional construction cost (a commonly used rate) and $20 \%$ of technical component cost. The latter rate is deemed reasonable in view of our recent and continuing experience in the construction and upgrading of the electr، $\mathrm{d}$-positron storage ring SPEAR.

Movable concrete shielding blocks, which will be used in some of the experimental halls, will be provided from existing supplies at LBL and SLAC and therefore are not included in the estimate. 
(\$ Thousands) ${ }^{1}$

ED\&I

Construction: Conventional Facilities

Site Work, Structures and Zuildings

Water and AC Electrical

3,950

B-2

Subtotal Conventional Facilities

15,350

Construction: Technical Components

Magnets

Magnet Power Supplies

Rf System

Vacuum System

Instrumentation and Control

Beam Transport System
5,350

450

5,000

5,700

2,800

3,050 A

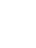

\section{Subtotal Technical Components}

Standard Equipment

22,350

50

Contingency $@ 15 \%$ on Conventional Facilities

and

e $20 \%$ on Technical Components

TOTAL

$\$ 53,350$

Escalation $9 \%$ per year

8,450

GRAND TOTAL

$\$ \underline{\underline{61,800}}$

${ }^{1}$ Cost estimate in FY1974 dollars. 
Engineering Design and Inspection

\section{(\$ Thousands) \\ Item Cost \\ Total Cost}

Corventional Plant (Sitework, Structures,

Water, Electrical) (Approximately 18\%

of Construction Cost Estimate of $\$ 15,350$ )

$\$ 2,800$

Technical Components (Approximately $2 i \%$ of

Construction Cost Estimate of $\$ 22,400$ )

4,650

TOTAL 
Sitework, Structures and Buildings

(\$ Thousands)
Item Cost $\quad$ Total Cost

Sitework

Roads (6,400 linear ft. @ $\$ 26.25 /$ linear $\mathrm{ft}$.)

Fencing (3, 300 linear $\mathrm{ft}$. $\$ 5.00 /$ linear $\mathrm{ft}$.)

$\begin{array}{ll}\text { Drainage } & 67\end{array}$

Sanitary Sewers $\quad 32$

Erosion Control $\quad 15$

Landscapiag

$\$ \quad 374$

Beam Housings

Bored Ring Sections $(2,827$

linear $\mathrm{ft}$. $\$ 837 /$ linear $\mathrm{ft}$.

and 656 linear ft. $\$ 1,800 /$

linear $\mathrm{ft}$.)

3,551

Ring Cut and Cover $(2,768$ linear

ft. @ $\$ 650 /$ linear ft.)

1,799

Ring Earthwork (150,000 cu. yds. (2) \$1.80/cu. yd.)

Ring Entrances (5 $\$ \$ 30,000$ ea.)

Beam Transport Tunnels and Shafts 569 
Schedule B-1 (cont'd)

( \$ Thousands)

Item Cost Total Cost

Interaction Areas

Site Preparation

$\$ \quad 537$

Structures (41, 630 sq. ft. @ avg. cost of $\$ 67.61 / \mathrm{sq}$. ft.)

2,815

Electrical and Mechanical

Cranes

254

$\$ 4,021$

Surface Buildings

Control Bullding Relocation and

Modification

$\$ 50$

Vacuum Shop and Power Supply

Building (20,000 sq. ft. (e)

$\$ 18.15 /$ sq. ft.)

Rf and I\&C Equipment Shelters

$(6,200 \mathrm{sq}$. ft. @ $\$ 35.32 / \mathrm{sq}$. ft. $)$

219

Cleaning Facility Building

(1,600 sq. ft. \& $\$ 21.25 /$ sq. ft.)

34

$\$ \quad 666$

TOTAL

$\$ 11,400$ 
Cooling Water Distribution System

Cooling Towers and Cooling

Tower Water Distribution

$\$ \mathbf{5 3 0}$

Magnet Cooling Water System 230

Rf Cooling Water System 153

Interaction A reas Cooling Water System

Vacuum Cooling Water System

Injector Cooling Water System 75

$\$ 1,600$

AC Electrical System

Rf System AC Services

\$ 495

Magnet Power Supplies AC Services 185

Water System AC Services

Ring, Injection and Interaction

Area Services

1,265

Emergency, Exterior Area Lighting and Construction Power 
Bending Magnets

Quadrupole Magnets

Correction Magnets

Bussing and Cooling Water

Supports

Installation, Magnetic Measurement, Alignment and Miscellaneous

TOTAL
$\$ 1,708$

1,077

343

812

555

855

SCHEDULE B-4

Magnet Power Supplies

Single-polarity Power Supplies

286

Bipolar Power Supplies

72

Installation

92

TOTAL

$\$ \quad 450$

SCHEDULE B-5

Rf System

Klystrons

$\$ 1,550$

Klystron Power Supplies

Rf Cavities

2,100

Rf Monitoring, Phasing and Control

500

Waveguide

$-200$

TOTAL

$\$ 5,000$ 
SCHEDULE B-6

\section{Vacuum System}

( $\$$ Thousands)

Item Cost

$\$ 2,370$

Ring Vacuum System

Interaction Areas Vacuum System

Cleaning Tanks and Niscellaneous

TOTAL
2,940

390

SCHEDULE B-7

Instrumentation and Control

Timing System

$\$ \quad 80$

Racks, Trays, Cables and Frames

Interlocks, Vacuum and Water

370

Magnets, Power Supplies and Electrostatic

Devices

Beam Monitors

Computers and Consol-

1,070

Video System and Communication

200

TOTAL

$\$ 2,800$

SCHEDUTE B-8

Beam Transport System and Injecti' in

Magnet Systems

$\$ 1,480$

Beam Line Instrumentation

305

Vacuum System

155

Inflection System

660

Insta!lation and Miscellaneous

450

TOTAL

$\$ 3,050$ 


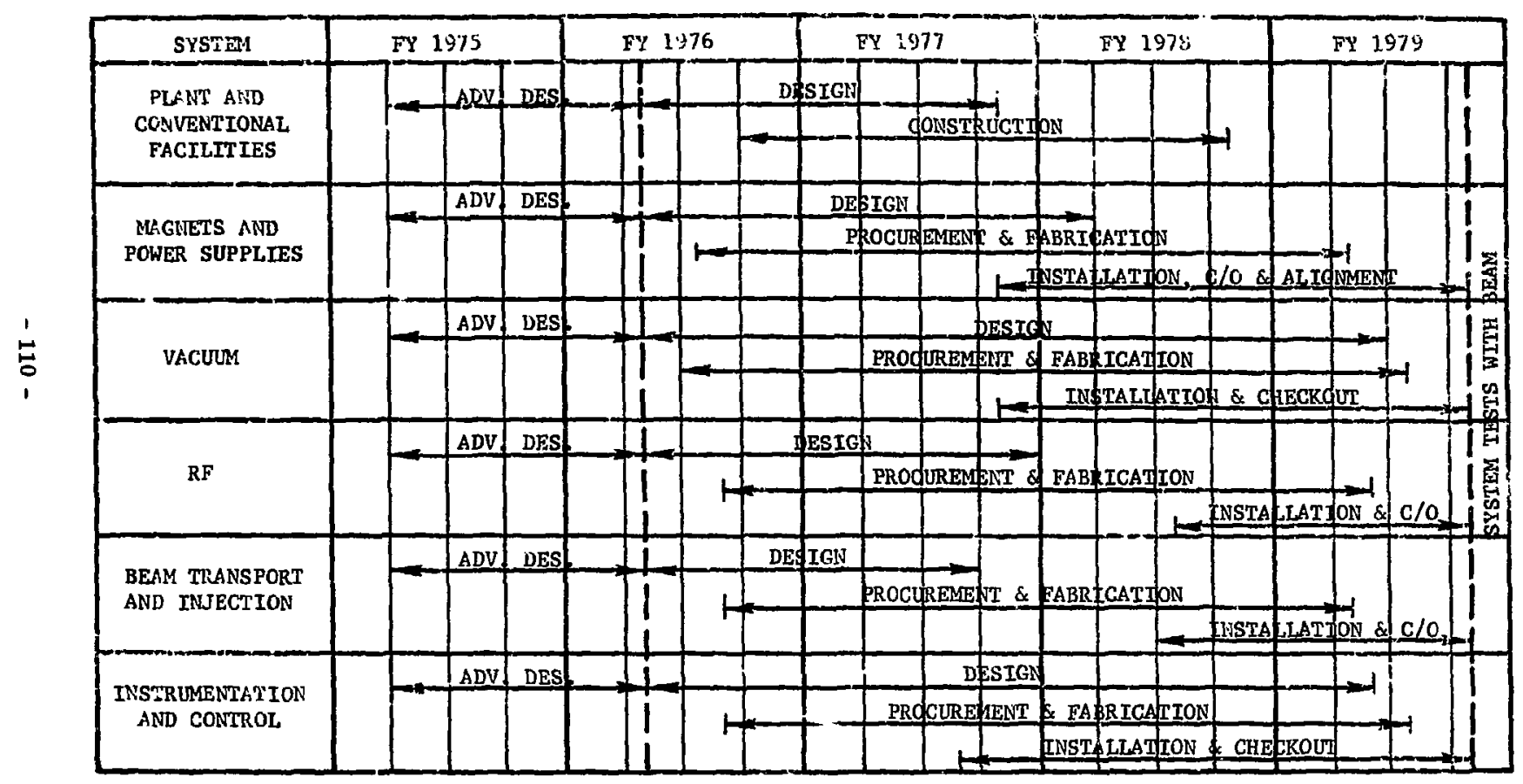




\section{APPENDIX A}

\section{The Proton-Electron System}

Introduction

The initial considerations ${ }^{25}$ for PEP included a scheme for achieving ultrahigh-energy collisions of electrons with protons as well as with positrons. In electron-proton collisions, PEP is capable of an enormous extension of parameters in traditional electron machine experiments (inelastic electron scattering, photoproduction, eks..), and in addition will open the field of weak interactions to practical experimentation with a well understood, well controlled probe-the electron.

This Appendix provides a brief description of a proton-electron system which utilizes the $15 \mathrm{GeV}$ electron ring of this proposal. Basically, a $200 \mathrm{GeV}$ superconducting proton storage ring is incorporated with the electron ring, within the same enclosure, to attain electron-proton collisions. The center-of-mass energy thus achieved is $110 \mathrm{GeV}$ which is the same as that which would be available with a $6000 \mathrm{GeV}$ beam from a conventional accelerator incident on a stationary hydrogen target (there is no economically feasible way of reaching these energies with a conventional accelerator). The facilities of the present proposal are designed to be compatible with this addition. The future electron-proton system, together with the presently proposed electron-positron system, would comprise a total facility of unique capability for particle physics research. Some of the details of this future step, along with the projected parameters, are outlined below. Physics Potential 26

a) Deep Inelastic Lepton Scattering $\left[e^{-}\left(e^{+}\right) p \rightarrow e^{-}\left(e^{+}\right)+\right.$hadrons $]$

Inelastic electron-proton scattering plays an essential and unique role in the investigation of the structure of the hadrons. The knowi: electromagnetic field generated by the scattered electron interacts with the local electromagnetic 
current of the proton and thus can probe the structure of the nucleon at arbitrarily small distances. This local interaction is in sharp contrast to hadron-hadron scattering in which the basic interaction between the particles is more complex. By varying the encrgy and angle of the scattered electron it is possible to "tune" or vary the virtual photon's mass $Q^{2}$ over a large range. In particular, it is possible to achieve virtual photon masses whose square is negative and whose magnitude is much greater than the proton mass and therefore allows for collisions in an asymptotic region not a ailable in accelerators using a fixed mass projectile.

Experiments on inelastic scattering at SLAC, where both the mass and energy of the virtual photon are large, have yielded profound and unexpected results. These resuits show that the cross sections do not depend independently on both the mass and energy of the photon, but instead on their ratio. This "scaling" behavior has led to major new concepts in our understanding of hadronic structure in terms of a possible substructure within the hadron that is composed of point-like constituents (partons). The greatly enhanced center-ofmass energy of a PEP facility would extend the measilrements of deep inelastic scattering far into the unknown region. With the example parameters used here the virtual photon energy would reach to $6000 \mathrm{GeV}$ and its mass to $110 \mathrm{GeV}$ compared to an energy of $20 \mathrm{GeV}$ and a mass of $5 \mathrm{GeV}$ at the present SLAC frontier.

Confirmation of the scaling behavior at these larger values of energy and mass would give support to these new ideas while observation of violations of scaling would indicate a new energy scale for hadronic phenomena perhaps associated with the production of new particles and of a "size" for the sonstituents themselves. This point is emphasized by the surprising results of the recent 
SPEAR experiments in the region of time-like momentum transfer which do not support these ideas of the quark-parton model, and in this respect make the further study of inelastic electron reactions even more intriguing. Other general and fundamental features io be studied for large photon masses include the applicability of Regge theory analyses, the validity of sum rules based on current algebra, and the "fragmentation" of very massive virtual photons into jets of secondary hadrons.

Besides the electromagnetic inelastic electron scattering, it will be possible to observe the effects caused by a weak neutral current of the type discovered in the recent CERN and NAL neutrino experiments. The effects of the neutral current would be observable as parity violations, charge conjugation violation, and possibly electromagnetic scaling violations. Since both energy and momentum transfer are easily determined, detailed knowledge as to the nature of these neutral current effects will be possible.

Thus, this unique feature of a PEP facility, the study of deep inelastic scattering, will yield results on one of the most significant problems in particle physics. b) Weak Interactions (ep $\rightarrow \nu_{e}+$ hadrong)

If the scaling phenomena observed in deep inelastic scattering is assumed to hold also for the weak interactions, as would be implied at least in part by the gonserved vector current (CVC) idea, then from the Fermi theory one is led to the conjecture that the total weak interaction cross section will continue to grow quadratically with the center-of-mass energy. This has the startling consequence that at energies in the PEP region the weak interactions, with their inherent violation of parity and strangeness, will have grown in strength to be comparable to the electromagnetic interactions. In fact in the regi on of the largest momentum transfer accessible with $200-\mathrm{GeV}$ protons and $15-\mathrm{GeV}$ electrons, the scaling hypothesis 
predicts that the deep inelastic electromagnetic cross section will be smaller than the weak process cross section.

Experiments with PEP will show either that the weak interaction ceases to be "weak" at high energies or that the Fermi theory in its simple form breaks down. The discovery of a failure in the Fermi theory would in itself be of the first magaitude in importance; additionally one could then entertain hopes of discovering the mechanism of breakdown.

\section{The Total PEP Concept}

PEP would provide a facility for high-energy $e^{-}-p$ and $e^{+}-p$ collisions in addition to $\mathrm{e}^{ \pm} \mathrm{e}^{-}$collisions. The electron-positron system is a straightforward extrapolation from lower energy storage rings, the properties of which are fairly well understood. On the other hand, there is no present experience with electron-proton systems, and the technical demands appear to be more formidable.

For the e-p system it is desirable, particularly in view of the weak interaction physics potential, to have the highest possible center-of-mass energy. For the prescribed size of the $15 \mathrm{GeV}$ electron ring, a proton energy of $200 \mathrm{GeV}$, corresponding to a center-of-mass energy of $110 \mathrm{GeV}$, can be reached with dipole field strengths of $44 \mathrm{kG}$, which is reasonably within the capability of superconducting techmology. Moreover, superconducting elements would facilitate substantial power savings compared to conventional magnet elements. For these reasons, a superconducting magnet system is selected for the proton ring.

The proton ring will be added within the same tunnel enclosure. In order to accommodate the present design, the electron ring would be moved in elevation, as shown schematically in Fig. A-1. The darker line in the figure represents the proton beam trajectory. The electron orbit, which has almost the same circumference, alternates above and below the proton ring. Short 


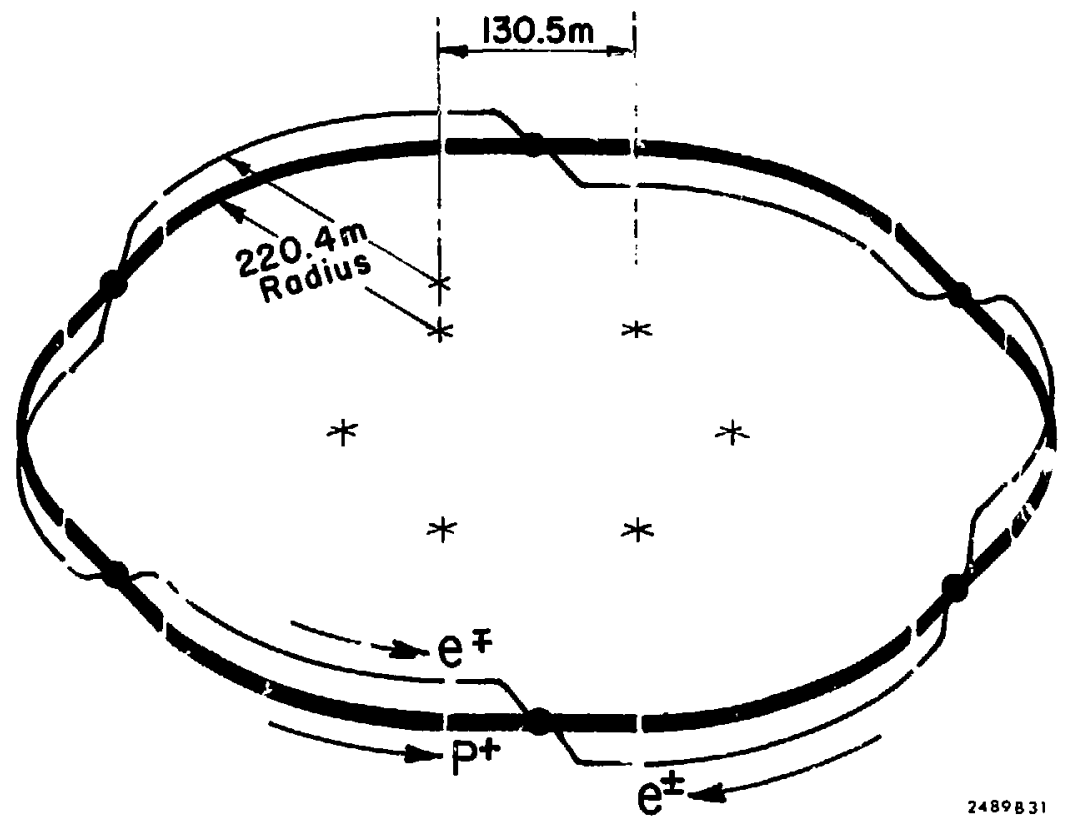

FIG. A1 Schematic view of the PEP electron-proton ring configuration. 
electron and proton bunches counter-rotate synchronously in their respective ring structures and collide in the six interaction regions at the points indicated by the large dots.

The orientation and depth of the tunnel in the preceding proposal provide adequate radiation shielding for the $200 \mathrm{GeV}$ proton ring, except, over experimental areas where additional shielding can be readily added in the future. The larger experimental areas are designed with the fiexibility to do future e-p experiments and are compatible with utilization of large detector systems envisioned for both $\mathrm{e}^{+}-e^{-}$and $e^{ \pm}-p$ reactions.

The possibility exists also for the addition of a second electron ring which would alternate in the opposite sense from the electron structure shown in Fig. A-1. This would provide for $\mathrm{e}^{+}-\mathrm{e}^{+}$and $\mathrm{e}^{-}-\mathrm{e}^{-}$collisions, as well as for higher luminosities for the $e^{+}-e^{-}$system at lower energies.

\section{Lattice System and Operating Parameters}

The alternating elevation of the electron $r$ ing $(F$ ig. $A-1)$ eliminates the need for a series of quadrupoles in the insertion region, which would otherwise be required to prevent objectionable vertical dispersion in the curved sections of the electron ring. As a result, the required insertions are shortened, thereby avoiding overcrowding of magnet elements in that region and providing useful space in which the circumfierence of the electron orbit may be altered to provide synchronization with the protons over a range of proton energies. For these reasons, this scheme with the alternating vertical configuration is believed preferable to fixed-elevation schemes for the electron ring which have been described previously. ${ }^{27}$

Protons will be injected into the superconducting ring at $5 \mathrm{GeV}$ from a conventional booster synchrotron, accelerated up to $200 \mathrm{GeV}$ and compressed 
longitudinally to the desired bunch length before injecting tine counter-rotating beam of electrons or positrons in the other ring. The electrons or positrons would be injected at $15 \mathrm{GeV}$ from the existing SLAC linac in a manner similar to that described in Section III $D$.

The insertion region, together with the neighboring cells of the normal cell structures, is shown in Fig. A-2. For this system, the only magnet elements common to both the electron and proton beams are doublets $\left(\varepsilon_{1,2}\right)$ at each end of the 20-m free space which includes the Interaction Point (I.P.), and the vertical bending magnets $\left(B_{1}\right)$. These doublets focus the electrons but have little effect upon the protons. The bending magnets $\left(B_{1}\right)$ direet the electrons up or down to the electron-ring structure. The small deflection given to the protons is compensated by three magnets $\left(B_{2,3,4}\right)$ which restore the protons to their median plane. $Q_{3,4}$ is the first principal quadrupole doublet of the proton system. Beyond $\mathrm{Q}_{3,4}$ is a $28-\mathrm{m}$-long straight section which is used to accommodate injection hardware and other beam components. It should be noted that there is more than sufficient space for these components such that the experimental region about the interaction point could be increased beyond $20 \mathrm{~m}$ if needed to install longer configurations if they are required by experiments.

The general lattice parameters of cell length, bend radius, ete., are identical to the electron storage-ring parameters given in Section III A. The length of the free space $(20 \mathrm{~m})$ in the interaction region represents a compromise between experimental requirements and orbit considerations. The vertical separation $(0.8 \mathrm{~m})$ of the electron and proton structures is sufficient to accommodate the magnets, rf cavities and injection components. Minimum separation is desired to reduce tunnel size and to minimize the power radiated by the electrons in the vertical dipoles. 


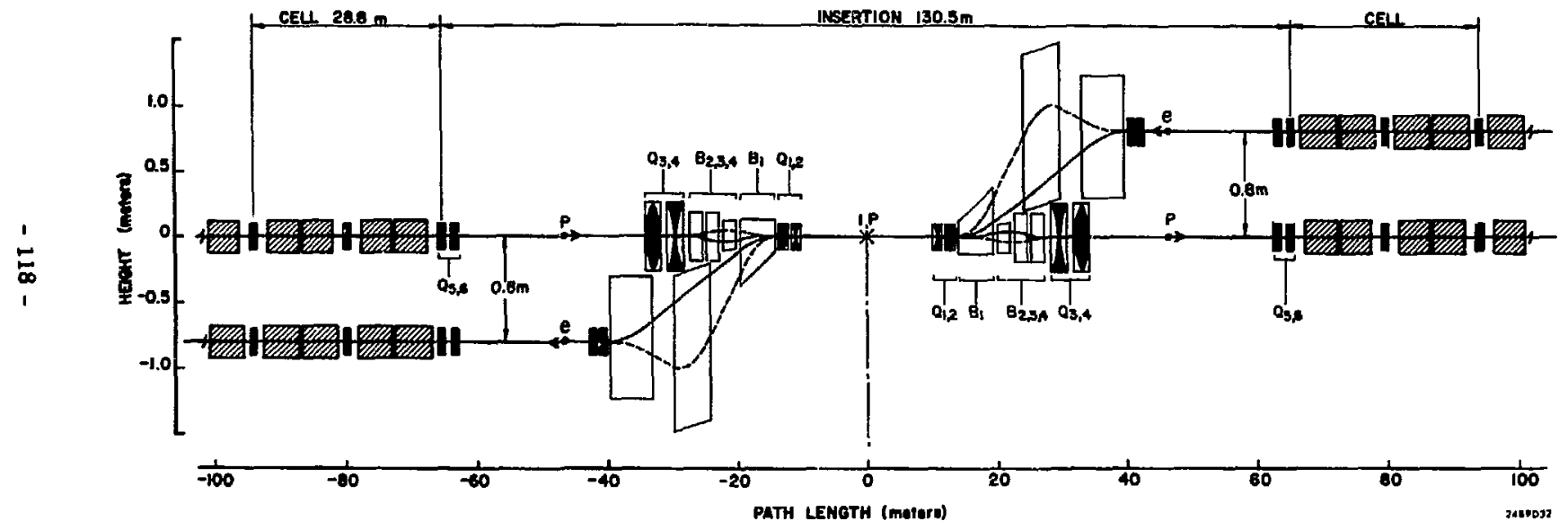

FIG. A2 Schematic side view of insertion and cell structure for the proton-electron system. 
Table Al shows a typical set of parameters for operation at $(200,15) \mathrm{GeV}$ and $(50,5) \mathrm{GeV}$. The variation of luminosity with energy cannot be scaled as readily as for electron-positron collisions, since the properties of proton beams scale differently with energy. Under the approximate constraint that the beambeam tune shifts should not exceed 0.005 for protons and 0.05 for electrons, and assuming that this effect is a performance limitation, the luminosity must decrease with decreasing energy - about a factor of 5 for the above practical range of energies.

The number of protons required (Table A1) is relatively modest - comparable to those planned for the NAL and CERN II synchrotrons. The number of electrons is about the same as that for the presently proposed storage ring - principally limited by the maximum radiated beam power. Both beams are divided into a large number of bunches to minimize the beam-beam effects as well as potential high-peak current effects.

The proton emittances are conservative, being somewhut larger than those currently achieved in the CERN PS or the Brookhaven AGS; there would be no advantage in smaller emittances because of the beam-beam effect. The absolute electron emittances are taken to be independent of energy for reasons already presented in Section III A.

The proton bunch length is as short as possible, consistent with moderate If voltage requirements, since high luminosity requires localizing the collisions to the low $-\beta$ region. In the electron ring, when sufficient rf overvoltage is provided to assure a long quantum lifetime, the natural electron bunch length is quite short enough to achieve the required localization.

The interaction point dlspersion is set to zero to avoid potential problems such as magnet ripple effects. In operation, it may be possible to improve the luminosity by going to finite dispersion at the interaction points. 


\section{Table A1}

Operating Parameters

Momentum
Number of Particles
Number of Bunches
Beam Power Fadiated
Luminosity/crossing
Momentum width - $\left(\frac{\Delta p}{p}\right)_{\text {rms }}$
Bunch Lengths ( $r m s)$
Interaction Point:
rms beam width
rms beam helght
$\beta$-function horizontal
$\beta$-function vertical
Diepersion

\begin{tabular}{lc}
$\mathrm{p}$ & 200 \\
$\mathrm{~N}$ & \multicolumn{2}{c}{3.6} \\
$\mathrm{n}_{\mathrm{B}}$ & \multicolumn{2}{c}{24} \\
$\mathrm{P}_{\mathrm{B}}$ & -25 \\
$g$ & \multicolumn{2}{c}{$10^{32}$} \\
$\sigma_{\mathrm{p}}$ & \multicolumn{2}{c}{.047} \\
$\sigma_{\mathrm{f}}$ & \multicolumn{2}{c}{25}
\end{tabular}

High-Energy Mode
Proton Electron

Low-Energy Mode
Proton Electron

$15505 \mathrm{GeV} / \mathrm{c}$

$0.7 \quad 3.6 \quad 1.06 \times 10^{13}$

4.9

$48 \quad 48$

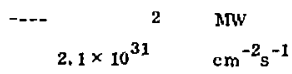

$.188 \quad .031 \%$

$\begin{array}{ccccc}.094 & .188 & .031 \% \\ 2.6 & 25 & 2.6 & \mathrm{~cm}\end{array}$

Normalized Emittance (95\% beam)

\section{Horizontal}

Vertical

Besm-Beam Tune Shift

Horizontal
Vertical

Betatron Function (maximum)

Chromaticity

$$
\begin{aligned}
& \text { Horizonlal } \\
& \text { Vortlcal }
\end{aligned}
$$

Betntron Tune

Dipale Fiold (Cella)

Quadrupolo Gradient (Cells)

Transition Gamma

$$
\begin{aligned}
& \beta \gamma \epsilon \epsilon_{x}^{/ \pi} \\
& \beta \gamma \epsilon_{y} / \pi
\end{aligned}
$$

.064
.021
.3 .25
1.42
0

.067

.012

.128

$.117 \mathrm{~cm}$

.043

$.021 \mathrm{~cm}$

$\beta_{\mathrm{x}}^{*}$

$\beta_{y}^{*}$

$\eta_{x}^{*}=\eta_{y}^{*}$

1.05

0.34

3.3

3.2 m

1.45

$1.05 \mathrm{~m}$

0

.016
.004

7.52
.752

.016

.004

2.51 cm-rad

$.251 \mathrm{~cm}-\mathrm{rad}$

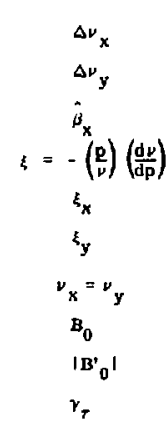

.002
.005

771

$\begin{array}{llll}4.1 & 3.6 & 4 & 4 \\ 3.7 & 5.0 & 4 & 4\end{array}$

$\begin{array}{llll}15.75 & 15.75 & 15.75 & 12.75\end{array}$

44.0

2.94

11.0

$.98 \quad k G$

602

53

140

$15.0 \mathrm{kC} / \mathrm{m}$

19.6

13. 2

14.5 
Machine Components

The characteristics and parameters projected for some of the principal machine components are described below.

Superconducting Magnet System. For the proton normal-cell structure, 192 dipoles are required, each 5 meters long, operating up io a maximum central field of $44 \mathrm{kG}$ at $200 \mathrm{GeV}$. There are 90 quadrupoles, each 1.2 meters long, with a maximum field at the conductor of $38 \mathrm{kG}$ for a maximum required gradient of $502 \mathrm{kG} / \mathrm{m}$. For these magnets the required good-field radius is 5.3 and $5.8 \mathrm{~cm}$ in the dipoles and quadrupoles respectively. The six insertion regions require, in addition, 24 quadrupoles of length 2.5 meters and 24 quadrupoles of length 1.4 meters. (The common doublet $Q_{1,2}$ and the bending magnets $\mathrm{B}_{1}$ and $\mathrm{B}_{2}$ will be conventional room temperature magnets.) The magnet design ${ }^{28}$ incorporates intrinsically stable, fine filament, NbTi superconductor that is well cooled with liquid helium and rigidly supported, and utilizes a simple compact cryostat-cryogenic transfer system. Operational reliability for large-scale superconducting systems needs to be established and is one of the major goals of the LBL research and development program and pilot accelerator program (ESCAR). ${ }^{29}$

Cross sections of the magnets are illustrated in Fig. A-3, and their location within the tunnel is shown in Fig. A-4. The ultra-high vacuum region is enclosed by a non-magnetic beam tube, upon which the multi-layered coil is wound. Circular symmetry is used in all inner regions to yield the best possible structural and magnetic properties.

The cryostat is continuous through a half-sextant of the ring with surrounding evacuated thermal insulation, an $80^{\circ} \mathrm{K}$ temperature shield and finally a room-temperature vessel. Helium at $4.4^{\circ} \mathrm{K}$ is introduced at the center of a 

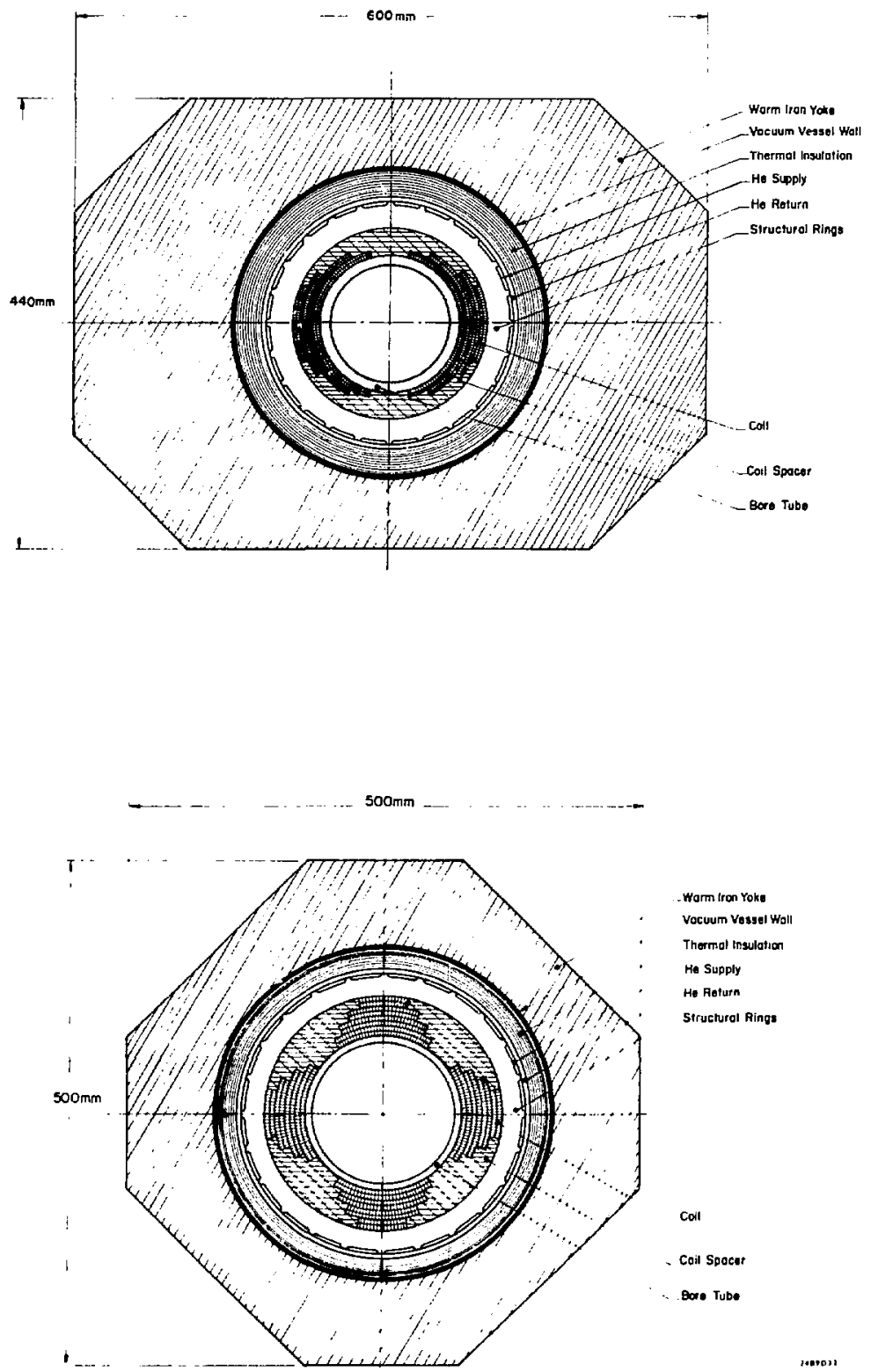

Wam iron Yok

Vocuum Vossel Wol

Thermol insulation

He Supply

He Rolurn

Struchural Rings

Coil

Cail Spocar

Done Tube

1901031

FIG. A3 Cross sectlons of the normal-cell superconducting dipole and quadrupole magnets. 

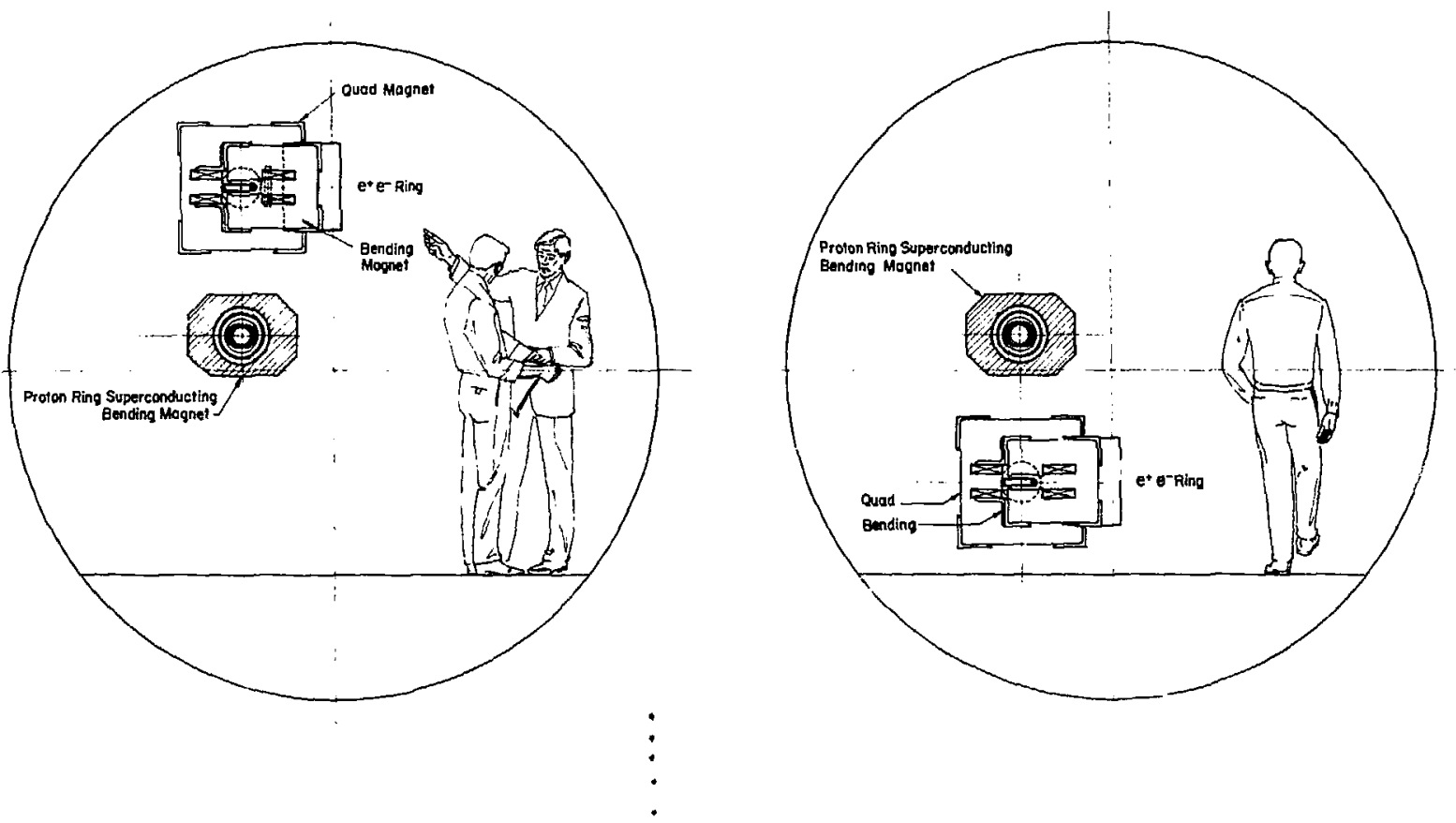

FIG. A4 Tunnel cross soctions with electron and proton magnets in alternate sextants of the ring. 
sextant and forced in both directions to the ends of the sextant. It then flows back at reduced pressure and temperature in the outer annular region of the cryostat, acting as a counter-flow heat exchanger. ${ }^{30}$ The only additional element in the tunnel is the transfer line from the sirface where the rest of the refrigeration equipment is housed. This scheme eliminates interference between refrigeration components and experimental apparatus near the insertion regions.

A summary of the expected heat loads is shown in Table A2. The heat leak at $4.4^{\circ} \mathrm{K}$ is assumed to be $1.2 \mathrm{~W} / \mathrm{m}$, and that from room temperature to $80^{\circ} \mathrm{K}$ is taken to be $2.5 \mathrm{~W} / \mathrm{m}$. Allowance is made for two pairs of electrical leads for the dipoles and six for the quadrupoles, which permits independent control of the quadrupoles adjoining the insertions. Additional allowance is made for quench leads and beam diagnostic and contrul equipment. In all, some $600 \mathrm{~kW}$ of installed power capacity will be required for each of the six refrigeration stations. The magnet power supply circu it will parallel the refrigeration system.

Table A2

A breakdown of the $4.4^{\circ} \mathrm{K}$ refriger ation required per super-period of the PEP proton ring

Heat leaks into the cryostat $420 \mathrm{~W}$

Electrical lead losses $220 \mathrm{~W}$

Cryogenic vacuum pumps

(in the interaction regions) $200 \mathrm{~W}$

Heating from the helium circulating pumps $260 \mathrm{~W}$

Total (steady state load) $1100 \mathrm{~W}$ Cooldown allowance $400 \mathrm{~W}$

Total installed refrigeration required $1500 \mathrm{~W}$ 
Vacuum System. A system which obtains pressures in the range of $10^{-11}$ to $10^{-12}$ Torr appears to meet the performance requirements. This vacuum can be obtained economically in the PEP proton ring by distributed cryopumps at $4.4^{\circ} \mathrm{K}$. This is the operating temperature of the superconducting magnets and provides practically unlimited pumping capacity for all gases except helium and hydrogen. Hydrogen can be pumped effectively by bare-surface cryopumps up to coverage of eight-tenths of a monolayer. The only troublesome helium gas loads which could arise come from leaks from the cryogenic refrigeration system. Heliur is aumped by bare surface cryopumps at a pressure of $10^{-11}$ Torr to only 1/1000 of a monolayer before saturation. Because of the large cold area of the superconducting magnet bores, however, about 200 minimum detectable leaks can be pumped effectively for four weeks.

The straight sections contain the accelerating cavities, the injection and extraction systems and the interaction regions with the cross-overs of the electrot-positron ring. These elements and synchrotron radiation will present additional gas loads for which the geometry and size of the cryopunps must be designed. Distributed cryopumping will be used where possitble. The hydrogen and helium pumping capacity of the straight section cryopumps can be increased as required by operation at a lower temperature by a permanent electro-deposit of porous silver or by a replaceable condensation deposit of $\mathrm{CO}_{2}$. The $\mathrm{CO}_{2}$ deposit could be especially useful in the valved cryopumps used for finish roughing and conditioning. These pumps could be operated steadily to remove small helium leak loads. Gas loads that could enter t.om the electron ring or pass from the straight sections into magnet cections will be intercepted by short lengths of tubul..r cryopimps that are easily cycled. 
Injection, Acceleration, and Bunching. A small $5 \mathrm{GeV}$ synchrotron is visualized as an injector for the superconducting ring. As the intensity and emittance requirements for PEP are not very stringent, there should be no difficulty in providing an economical system.

The present concept for achieving the desired proton bunches is as follows. Each of the 24 bunches is successively loaded from separate booster pulses into existing buckets in the main ring. The bunch lengths are such that they would fit comfortably in buckets at harmonic number $96(4 \times 24)$. For a longitudinal emittance of $10 \pi \mathrm{cm}$, in $(\Delta \beta \gamma, \Delta Z)$ units, the holding voltage would be about $400 \mathrm{keV} / \mathrm{turn}$. This same voltage would be sufficient for an acceleration time of 10 seconds or more, and the normal damping would produce a bunch length at $200 \mathrm{GeV}$ which would fit $a$ bucket at four times higher frequency ( $h=384$, $f=53 \mathrm{MHz}$ ). At this frequency the voltage would be raised to compress the bunches to the desired length, the final value being $\sim 6 \mathrm{MeV} / \mathrm{turn}$ if the emittance is preserved throughout.

The rf structure is visualized as consisting of a number of tubes which would act as drift tubes at the initial frequency, and as half-wave lines at the final frequency. For the system cited above, 17 tubes, occupying a total length of 50 meters would be utilized with a power sonsumption of $\sim 2 \mathrm{MW}$.

In Fig.A2, it is seen that there are twelve straight sections of $28 \mathrm{~m}$ length in the proton ring befween $Q_{3,4}$ and $Q_{5,6}$ and twelve corresponding straight sections in the electron ring. Three sections are used for electron, positron and proton injection. The remaining nine sections are available for electron and protrin rf systems, beam disposal systems and diagnostics.

The brief description of components and parameters in this Appendix does n. it represent the results of an optimized system or a comprehensive analysis 
of the components, but rather is a conceptual analysis to help insure that a future e-p system is feasible and compatible with the present proposal for an electron-positron system. In particular, there are numerous orbit dynamics questions, both single-particle and collective, which require further study; also, substantial development is needed for assurance that super-conducting systems are operationally viable. 


\section{APPENDIX B}

\section{Summary of Soil Studies to Date}

A soil studies program was undertaken in the summer of 1973. A $2100 \sim m-$ circumference oval encircling the SLAC beam switchyard and the present Research Area was examined. The first phase of this program was a soil study in which seventeen holes around the periphry of this $2100-\mathrm{m}$ oval were drilled, logged and sampled with a truck-mounted $15-\mathrm{cm}$ auger. The exploratory holes indicated a rather heterogeneous mixture of gravel, sand, clay, claystone and shale near the surface throughout the area with firmer, more competent sandstone being encountered 6 to 20 meters below the surface. Eight of these seventeen holes are within 10 meters of the present PEP ring alignment. The other nine holes vary from 30 to 60 meters from the present ring alignment. The nominal elevation of the relatively competent sandstone is approximately 70 meters above mean sea level.

The second phase of this soil study program was the performance of refraction geophysical surveys to determine the depths to various geologic units with different compressional wave velocities. A layer was detected just below the surface with a relatively low compressional wave velocity of between 360 and 540 meters per second. The low wave velocity indicates dessicated adobe and sandy clay. An underlying layer with wave velocities varying from 700 to 1000 meters per second is composed of differentially weathered, partially decomposed, very fine-grained sancistone, shale and clay with gravel. It is postulated that, based on experience elsewhere in the SLAC project with earth materials of 11ke origin end composition, the 700-to 1000-meters-per-second material conservatively sas an allowable bearing value of at least 20,000 kilograms per square meter. Below this layer, generally at depths in excess of 
6 meters below the ground surface, relatively competent earth materfal is present with a compressional wave velocity in excess of 1500 meters per second.

The general correlation between the drill holes and the refraction geophysical surveys indicates that, at an average elevation of 70 meters, at which drilling became difficult, a geologic unit of compressional wave velocity of 700 to 1000 meters per second exists and that, below this elevation, a geologic unit of greate: harchess and competence is gradually entered. 
APPENDIX C

CCCPERATIVE SASIC AGREEMEIT

BETHEEN

THE BOARU OF TRLSTEES OF THE LELAid SIANFORD JUNIOR URIVERSITY

AND

THE REGEITSS OF THE UNIYERSITY OF CALIFORIIA

This Ccoperative Basic Agreer.ent is entered into effective this $\hat{s}^{\text {th }}$ day of February, 1974, by and between the 6OARD OF TRUSTEES OF THE LELAiD STAHFORD UUIiOR LiNIVERSITY (hareinafter called "Stanford") and THE REGENTS OF THE UHIVERSITY OF CALICORNIA (hereinafter called "UC").

\section{RECITIILS}

I. IITRODUCTIOit

A. The Ernest Orlanis Lawrence Berkelcy Laboratory (LBL) is a ilationa)

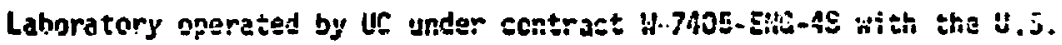
Atonic Energy Cormission (AEC). The Director of LBL reports to ine President of $U C$. The Stanford Linear Accelerator Center (Si-iC) is a National Facility for High Energy Physics Research operated by Stanioid under contract AT(04-3)-515 with the AEC. The Uirestor of SLAC reports to the Presicient of Stanford.

B. For several years LBL and SLAC have been ccilaborating in the study of a novel high-energy particle accelerztor system consisting of a ring containing counter-rotating persistent beams of eleibrons and jositrons, and a second intersecting ring containing a persistent rotating beam of

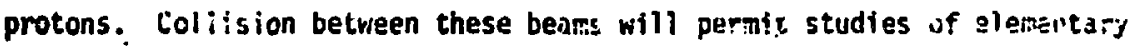
particles at higher energy, and tnerefore in finer detail tinan ever jefore. 
C. The concept of the system, called PEP for "proton-electron-positron," has been developed to the point where design and construction of an accelerator can take place. It appears desirable to accomplish the realization of this concept in stages. The first stage will consist of a single ring of approximately 300 meter radius able to contain counter-rotating beams of electrons and positrons with energies up to $15 \mathrm{GeV}$ at full interaction rats. These particles will be injected In:o the ring by the existing linear accelerator at SLAC. Provision vill be made in the construction of PEP to maintajn full compatibility with the later addition of a superconducting proton ring for protons of energies up to about $200 \mathrm{Gel}$ and/or a second electron ring to provide for collision of electrons with electrons and positrons with positrons. Prosision will also be made for the future Installation of experimental facilities for the fuil PEP Project. Meanwhile, the single ring will comprise a unique tool for corducting experiments involving electron-positron collisions, and by itself will be the basis for a front line experisiental program for nany yeurs. For ease of reference, the tel'm "PEP Project" as used nereafter in this Agreement means the design, construction and operation of the PEP acceierator system as finally funded, built, and cperated.

D. In corisideration of the iureyoing facts, and being keenly aware of the potentialities of the PEP Project for advancing mankind's knowlefge of the censtitution and natire of mattur, and in appreciation that tie task is of such challenge as to warrant the marshalling of the contined 
Cooperative Basic Agreement Stanford/UC -3-

resources and talents of Stanford and $U C$, with a consequenc sense of the historical importance of this act the parties hereto enter into this Cooperative Basic Agreement.

\section{AGGEEMENTS}

\section{COISSTRUCTIOHI PROPOSAL}

A. It is agreed that LBL and SLAC will collaborate in the preparation of - construction proposal to be submitted to the Atomic Ener yj Commission for conmencement of construction in Federal fiscal year 1976 of a high energy electron-positron ring to be coristructed at SLAC and to be operated jointly by SLAC and LBL as a national physics facility as described in Articles III. and J". of this agreement. Provision will be made in the construction of PEP to maintain full compatibility with the later addition of a superconducting proton ring for protons of energies up to about $200 \mathrm{GeV}$ and/or a sacond electron ring to frovide for collision of electrons with electrons and positrons with positrons. Provision will also be made for the future installation of experimental facilities for the fuli pef Project.

B. The technical and scientific justification for this construction proposal will be based specifically on the electron-positron collir. ing beam device. The parties agree that both scientific interest and current technical knowledge are such as to justify the construction of an electrori-positron storage ring in its own right; they also emphasize that the scientific goals of the full fEP Project provide good reasons to include in the design trose features essential to permit realization of the full PEP Project in the future. 
Cooperative Basic Agreement Stanford/UC -4-

C. As contained in the "5-year Budget Assumption" for 1976-1980 which was submitted by LBL and SLAC on November 13, 1973 to the AEC, the construction cost of the first stage of the PEP Project is estimated to be between $\$ 50$ and $\$ 60$ million.

\section{MANAGEIAENT PLAiV}

A. It is agreed that the goal of the project management is a true collaboration between LBL and SI.AC, the PEP Project will have a senior scientific and technical staff drawn in a balanced way from the two Laboratories. These persons, while working on the PEP Project, will remain employees of their respective Laboratories. In particular the Project Jirector and Deputy Director will be appointed by tine Directors of LSL and SLAC so that one comes from each Laboratcry. The Project Director will report to the Directors of LBL and SLAC.

8. The two Laboratory Directors will be advised in relation to the profect by a single PEP Program Comittee (PPC) having a majority of non-SLAC, non-LBL menibers. This Comittee will be appointed jointly by the Uirectors of the Laboratories. This Comittee shall advise the Directiors on all major phases of the PEP Project such as experimental facilities construction, and storage ring modification projects. Such projects shall go forward only after authorization by both Laboratory Directors, irrespec:ive of the source of funding. The Comittee shall meet as often as required but not less frequentiy than twice each year. 
C. It is intended that the PEP Project will be operated as a National Physics Facility. Steps are in progress to involve the national high-energy-physics community in planning for the physics utilizaticn as well as in setting up appropriate advisory mechanisms. In particular, it is agreed that an Experimental Program Committee (EPC) vill be constituted to reviaw experimental proposals under procedures designed to assure equitable access to the entire high-energy physics community. Jetailed scheduling decisions and coordination of PEP Project experiments with SLAC operations shall be made under SLAC procedures.

D. The Presidents of Stanford and $J C$ will be advised of the status of the PEP Project through, respectively, the Directors of SLAC and L8L. In addition, the Scientific Policy Comnitiee (SPC) of SLAC, advisory to the President of Stanford University, and the Scientific Educationai Advisory Committee (SEAC), advisory to the President of the University of California, will schedule joint meetings when deened advisable if it is believed that further advice on scientific policy governing the conduct of the PEP Project should be made avallable to the Presidents of the collaborating universities and, through them, as appropriate, to the Atomic Energy Cormission.

E. Stanford and UC may enter into modifications of this agreenent or into agreements implementing this agreement as may be necessary to carry out the PEP Project. 
Cooperative Basic Agreement Stanford/UC $-6-$

\section{HANPOWER AIID FISCAL ARRAIIGEMEIITS}

A. Preconstruction research and development work is now under way at both Laboratories, supported by the operating funds of each institution under the financial plans of their contracts with AEC.

B. The construction proposal described in Article II. A. will set forth the scope and estimated costs of major elements and components of the design and construction phase of the first stage of the PEP Project.

C. It is agreed that Stanford and UC shall seek to have the construction of the first stage of the PEP Project performed by Stanford under a contract between Stanford and the Atomic Energy Commission. Requests to the Atomic Energy Commission for construction directives to proceed with stages of the construction of the PEP Project sha?l be approved by both Laboratory Birectors. It is agreed that the health and strength of the technical high-energy support efforts as well as of the research programs of the two Laboratories must be maintained, and this goal will be used as a guide in determining how the personnel and facilities of each Laboratory are to support the design, preparation of specifications, and construction of the PEP Prcject.

D. After completion of construction to the satisfaction of both Laboratory Directors, SLAC and LBL will participate in the operation of the PEP Fac1lity as follows: 
1. SLAC will be responsible under the terins of Article III. for managing and coordinating accelerator operations. Funding support for accelerator operations will be through the SLAC operating contract with AEC.

2. The operation of each experimental facility built with SLAC and/or LBL funds shali be the responsibility of SLAC or LBL and shall be supported with their operating funds in the manner most advantagecus to the conduct of the national high-energy physics program.

3. During the operational phase of PEP, accelerator research ind development and faciiities research and development pertaining to PEP, as we!l as proconstruction work for the full PEP, will be supported by the two Laboratories, using their operation funds.

4. Experimental facilities construction and PEP storage ring modification projects may be supported separately or jointly in accordance with funding provided to LBL and SLAC.

5. The Laboratcries will participate in the physics research program of the PEP Project using operating funds from their separate contracts in a manner similar to that of other laboratories or universities performing research at the PEP National Physics Facility. 


\section{SETTLEMEIT OF OISAGREEMEIVTS}

Should the two Laboratory Directors be unable to agree on a substantive issue relating to this Agreement, it shall be referred to the Presidents of Stanford and uC for resolution.

\section{TERM AND TERMINATION}

This Agreenent shall continue in force from year to year from date of execution; provided, however:

A. Should Federal or otiner funding not be obligated for the construction of the PEP Project by June 30,1980 , this Agreement shall terminate.

B. This Agreement shall continue so long as Federal or other funding is provided for the op:ration of SLAC and LBL and for the construction and operation of the PEP Project.

IN WITIESS WHEREOE, the parties hereto have executed this Agreement as of the date first above written.

THE REGENTS OF THE

UNIVERSITY OF CALIFORNIA

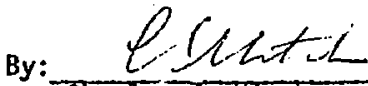

Charles J. Hitch

President, Liriversity of

California

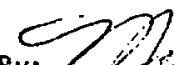

Marjoric o. hijolinari

Secretary

By:

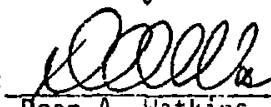

Dean A. Watkins

Chairman
THE BOARD OF TRUSTEES

OF THE LELAIV STAIYFORD

JUNIOR UNIVERSITY

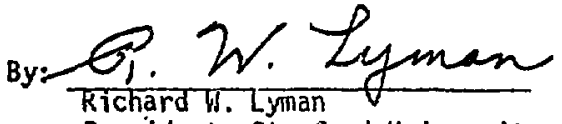

President, Stanford University

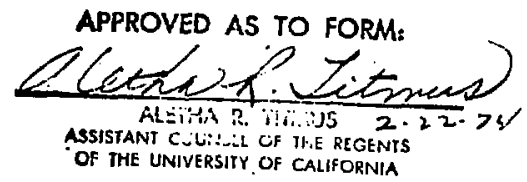




\section{RE FERENCES}

1. C. Pellegrini et al. , Proceedings of the 8th International Conference on High Energy Accelerators - CERN 1971 (CERN, Geneva 1971) p. 153.

2. T. Elioff, IEEE Trans. Nucl. Sc1. NS-20, No. 3, 1039 (1973).

3. R. Bangerter et al., IEEE Trans. Nucl. Sci. NS-20, No. 3, 786 (1973).

4. M. Briedenbach et al., Phys. Rev. Letters 23, 935 (1969). R. Taylor in Proceedings of the Fourth International Symposium on Electron and Photon Interactions at High Energies, Liverpool, England, September 1969 .

5. A. Litke etal., Phys. Rev. Letters 30,1185 (1973). See also Review Faper by K. Strauch, Proceedings of the 1973 International Symposiunn on Electron and Photon Interactions at High inergies, Bonn, August 1973.

6. Invited paper presented to Conference on Lepton-Induced Reactions at Irvine, California, December 1973, presented by B. Richter.

7. See, e.g., T. Appelquist and H. Georgi, to be published, and earlier work by H. Politzer, Phys. Rev. Letters 30, 1346 (1972).

8. Y. Nambu in Preludes in Thooretical Physics, North Holland, Amsterdam, 1966.

H. Fritzsch and M. Gell-Mann, Proceedings of the 16th International Conference on High-Energy Physics, University of Chicago and NAL (1972), and H. Lipkin, Phys. Rev. D7, 1850 (1973).

9. See, e.g., Review Paper by J.D. Bjorken in Proceedings of the 1973

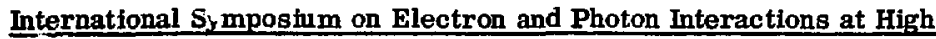
Energles, Bonn, August 1973.

10. S. D. Drell, D. Levy and T. M. Yan, Phys. Rev, D1, 1117 (1970).

11. F.W. Busser et al., Phys. Letters 46B, 471 (1973). 
12. N. Cabibbo and R. Gatto, Phys. Rev. 124, 1377 (1961).

13. F.J. Hasert et al., Phys. Letters 46B, 138 (1973).

14. A. Benvenuti et al., to be published in Phys. Rev. Letters.

15. See, e.g., S. Weinberg, Phys. Rev. Letters 19, 1264 (1967), 1688 (1971).

16. V.N. Baier, Usp. Fiz. Nauk 105, 441 (1971), and references therein.

17. Se€, e.g., J. Godine and A. Hankey, Phys. Rev. D6, 3301 (1972);

V. Cung, A. Mann and E. Paschos, Phys. Letters 41B, 355 (1972).

18. S.J. Brodsky in Proceedings of the 1971 International Symposium on

Electron and Photon Interactions at High Energies, Cornell University, August 1971.

19. S.J. Brodsky, T. Kinoshita and H. Terezawa, Phys. Rev. Letters 25, 972 (1970), Phys. Rev. D4, 1532 (1971).

20. M. A. Allen and R.A. McConnell, IEEE Trans. Nucl. Sci. NS-20, No. 3, 373 (1973).

21. E.A. Knapp, B. C. Knapp and J.M. Potter, Rev. Sci. Instr. 39, 979 (1968).

22. F. Amman, IEEE Trans. Nucl. Sci. NS-20, No. 3, 858 (1973).

23. Cummings et al., J. of Vac. Sci. and Tech. $\underline{8}, 348$ (Jan. -Feb. 1971).

24. Carmin and Pellegrin, Nucl. Inst. and Meth. 113, 423 (1973).

25. C. Pellgrini, J. Rees, B. Richter, M. Schwartz, D. Möhl, and A. Sessler, Proceedings of the 8th International Conference on High Energy Accelerators, CERN, 1971, p. 153.

26. LBL-SLAC Study Group, LBL Report 750 or SíAC Report 146, April 1972.

27. R. Bangerter, A. Garren, P. Morton, J. Rees, and L. Smith, Proceedings of the 1973. Particle Accelerator Conference, San Francisco, March 5-7, 1973, p. 786. 
28. W. Gilbert, R. Meuser, and F. Toby, Proceedings of the International Conference on Magnet Technology, BNL, New York, Sert. 19-22, 1972, and W. Gilbert, LBL-2418, Nov. 1973.

29. LBL Report P-1, Sept. 1973.

30. "Refrigeration Study for the NAL Fnergy Doubler," Cryogenic Technology Incorporated, Jan. 25, 1974 (unpublished). 